\title{
New definitions of old symmetries in Little Higgs and Supersymmetric models
}

by

\author{
Claudia Frugiuele
}

\begin{abstract}
A thesis submitted to the Faculty of Graduate and Postdoctoral Affairs
in partial fulfillment of the requirements for the degree of

Doctor of Philosophy

in

Physics
\end{abstract}

Ottawa-Carleton Institute for Physics

Department of Physics
Carleton University
Ottawa, Ontario, Canada

September 17, 2012

Copyright (C)

2012 - Claudia Frugiuele 
Library and Archives

Canada

Published Heritage

Branch

395 Wellington Street

Ottawa ON K1A ON4

Canada
Bibliothèque et

Archives Canada

Direction du

Patrimoine de l'édition

395 , rue Wellington

Ottawa ON K1A ON4

Canada
Your file Votre référence

ISBN: 978-0-494-93672-6

Our file Notre référence

ISBN: $978-0-494-93672-6$
NOTICE:

The author has granted a nonexclusive license allowing Library and Archives Canada to reproduce, publish, archive, preserve, conserve, communicate to the public by telecommunication or on the Internet, loan, distrbute and sell theses worldwide, for commercial or noncommercial purposes, in microform, paper, electronic and/or any other formats.

The author retains copyright ownership and moral rights in this thesis. Neither the thesis nor substantial extracts from it may be printed or otherwise reproduced without the author's permission.
AVIS:

L'auteur a accordé une licence non exclusive permettant à la Bibliothèque et Archives Canada de reproduire, publier, archiver, sauvegarder, conserver, transmettre au public par télécommunication ou par l'Internet, prêter, distribuer et vendre des thèses partout dans le monde, à des fins commerciales ou autres, sur support microforme, papier, électronique et/ou autres formats.

L'auteur conserve la propriété du droit d'auteur et des droits moraux qui protege cette thèse. $\mathrm{Ni}$ la thèse ni des extraits substantiels de celle-ci ne doivent être imprimés ou autrement reproduits sans son autorisation.
In compliance with the Canadian Privacy Act some supporting forms may have been removed from this thesis.

While these forms may be included in the document page count, their removal does not represent any loss of content from the thesis.
Conformément à la loi canadienne sur la protection de la vie privée, quelques formulaires secondaires ont été enlevés de cette thèse.

Bien que ces formulaires aient inclus dans la pagination, il n'y aura aucun contenu manquant. 


\section{Abstract}

The Standard Model of particle physics is an extremely successful theory that describe almost all experimental data in particle physics today. However it remains incomplete and leaves many question unanswered. In this thesis we study two classes of models aimed at understanding the physics of electroweak symmetry breaking: supersymmetric models and little Higgs models. In the first Chapter of this thesis a brief introduction to the Standard Model is given together with an explanation of the main motivations for physics beyond the Standard Model. Chapter 2 contains original work on Little Higgs models based on [1] . We introduce a new definition of a discrete $T$ parity which is compatible with a strongly interacting UV completion of these models. The old definition of this symmetry has been proven to be anomalous and this would rule out the possibility of having a dark matter candidate. We implemented this new symmetry in a $S U(6) / S p(6)$ models and we study its dark matter candidate. In Chapter 3 we give a general introduction to supersymmetry and to the Minimal Supersymmetric extension of the Standard Model (MSSM). We summarize the main aspects of models with Dirac gauginos and with a $U(1)_{R}$ symmetry which is the main topic of the rest of thesis. In Chapter 4 we introduce our model [2] where the $R$ symmetry is identified with one of the lepton number and where the sneutrino plays the role of the down type Higgs. We explore bounds on this model coming from electroweak precision measurements, and neutrino masses, and we show that the parameter space is still large. Finally we present a generalization of the model 
where the $R$ symmetry is identified with the global lepton number, [3]. In Chapter 5 we explain how the correct masses and mixing angle of neutrinos can arise from the necessary $R$ breaking from the cosmological constant cancellation, [3]. Finally in Chapter 6 we present a work in progress where we study the phenomenology of our model at the LHC. 


\section{Acknowledgments}

First of all I would like to thank my advisor, Thomas Grégoire, for his guidance and his support. Thank to my collaborators Enrico Bertuzzo, Eduardo Pónton and Piyush Kumar. I am also thankful to Tilman Plehn, and Stefano Forte. Thank to the particle physics group at Carleton and to Eva Lacelle. I finally thank the NSERC for the financial support, and the University of Edinburgh for the financial support while I was a student there. 


\section{Preface}

The original work in the thesis is contained in Chapter 2, 4, 5, and 6 .

Chapter 2 is based on a paper done in collaboration with Tom Brown and Thomas Grégoire, [1]. Section 2.6 of Chapter 2 is based on work done by Tom Brown under the supervision of Thomas Grégoire, the rest of the work was done only by myself and Thomas Grégoire.

Chapter 4 is based on two papers: the first one [2] done in collaboration with Thomas Grégoire and the second [3] with Enrico Bertuzzo. Chapter 5 is based on [3]. Chapter 6 contains original work done in collaboration with Thomas Grégoire, Piyush Kumar and Eduardo Pónton. A paper is in preparation. 


\section{Table of Contents}

$\begin{array}{lll}\text { Abstract } & \text { ii }\end{array}$

$\begin{array}{lll}\text { Acknowledgments } & \text { i }\end{array}$

$\begin{array}{ll}\text { Preface } & \text { ii }\end{array}$

Table of Contents $\quad$ iii

List of Tables $\quad$ vii

List of Figures $\quad$ viii

1 The Standard Model and Beyond $\quad 1$

1.1 The Standard Model . . . . . . . . . . . . . . . . 2

1.1.1 Gauge symmetry ................... 3

1.2 Higgs Mechanism ...................... 5

1.3 The Standard Model . . . . . . . . . . . . . . . . . . 7

1.4 Physics Beyond the Standard model . . . . . . . . . . . . . . . 10

1.4.1 Dark matter ................... 11

1.4.2 Theoretical motivation for BSM physics ......... 11

1.5 The Hierarchy problem and its solutions . . . . . . . . . 12

1.5.1 The Hierarchy problem . . . . . . . . . . . . 12

1.5.2 Little Higgs models . . . . . . . . . . . . . . . 13 
$1.5 .3 \quad$ Supersymmetry $\ldots \ldots \ldots \ldots \ldots \ldots$

2 UV friendly T parity in the $S U(6) / S p(6)$ Little Higgs model $\quad 16$

2.1 Little Higgs models, $T$ parity and strongly coupled UV completion . . 16

2.1.1 T-parity in the Littlest Higgs model . . . . . . . . . . . 19

2.2 The $\mathrm{SU}(6) / \mathrm{Sp}(6)$ model with T-parity $\ldots \ldots \ldots \ldots$

2.2 .1 Global symmetries . . . . . . . . . . . . . . 20

2.2 .2 Gauge interactions . . . . . . . . . . . . 22

2.2 .3 Top sector $\ldots \ldots \ldots \ldots \ldots \ldots \ldots \ldots$

2.2 .4 Light Fermions . . . . . . . . . . . . . . . . . . . . 29

2.3 Radiative corrections . . . . . . . . . . . . . . . . 31

2.3 .1 One-loop quadratic divergence . . . . . . . . . . . 32

2.3.2 One-loop $\log$ divergence $\ldots \ldots \ldots \ldots \ldots$

2.3.3 One-loop finite correction $\ldots \ldots \ldots \ldots$. . . . . . . 34

2.4 Electroweak symmetry breaking . . . . . . . . . . . . 35

2.5 Electroweak Precision Observables . . . . . . . . . . . . . . 38

2.5 .1 Higgs sector contributions $\ldots \ldots \ldots \ldots$

2.5.2 Fermionic sector contributions $\ldots \ldots \ldots \ldots$

2.6 Dark Matter . . . . . . . . . . . . . . . . . . . . . . . 41

2.7 Phenomenology . . . . . . . . . . . . . . . . . . . . . . 43

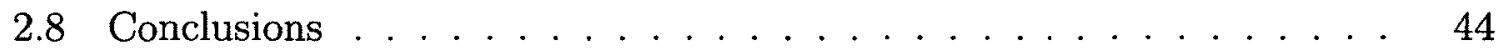

$\begin{array}{lll}3 & \text { Supersymmety } & 47\end{array}$

3.1 Supersymmetric algebra, Superspace and Superfields . . . . . . . 47

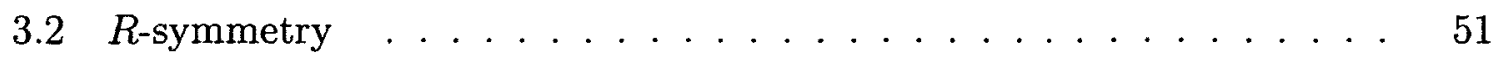

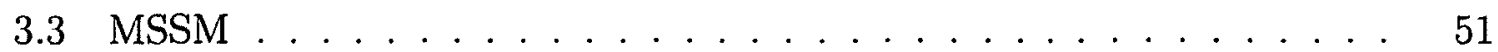

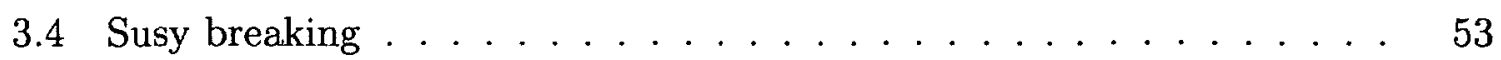

3.4.1 MSSM soft SUSY breaking terms . . . . . . . . . 55 
3.5 Natural MSSM and the LHC . . . . . . . . . . . . . 56

3.6 Dirac gauginos and Supersoft Supersymmetry breaking . . . . . . 57

3.6.1 Supersoft terms and natural SUSY . . . . . . . . . 60

3.6.2 $U(1)_{R}$ symmetry: another motivation for Dirac gauginos . . . 61

3.7 Models with a quasi exact $R$-symmetry $\ldots \ldots \ldots 62$

3.7 .1 The MRSSM . . . . . . . . . . . . . . . . 63

$4 \quad R$ symmetry as the lepton number $\quad 65$

4.1 Making the sneutrino a Higgs with a $U(1)_{R}$ lepton number . . . . . 65

4.1.1 Electroweak symmetry breaking . . . . . . . . . . . . . . . 69

4.1 .2 Lepton mixing . . . . . . . . . . . . . . . . . 73

4.2 Constraints from electroweak precision measurement . . . . . . 74

$4.3 \quad R$-symmetry breaking . . . . . . . . . . . . 79

4.3 .1 Neutrino masses . . . . . . . . . . . . . . . . . 79

$4.4 R$-symmetric gauge mediation $\ldots \ldots \ldots \ldots$

4.4.1 $R$ symmetry, and the $\mu / B_{\mu}$ problem $\ldots \ldots \ldots 81$

4.4.2 Yukawa coupling for lepton $a \ldots \ldots \ldots \ldots$

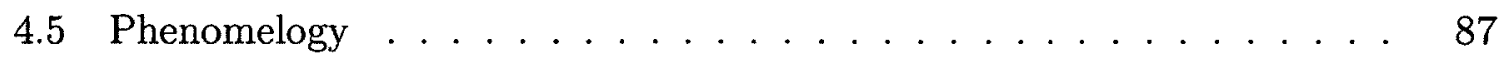

4.5 .1 MMRSSM at the LHC . . . . . . . . . . . . . 87

$4.6 U(1)_{R}$ as global lepton number $\ldots \ldots \ldots \ldots$

4.6 .1 The model . . . . . . . . . . . . . . . . . . . . . . 89

4.6.2 Electroweak precision measurements and flavor constraints . . 94

4.7 Lepton Yukawa coupling from SUSY breaking $\ldots \ldots \ldots \ldots$

4.7.1 An example of Yukawa sector $\ldots \ldots \ldots \ldots$

5 Fitting neutrino physics with $U(1)_{R}$ lepton number 101

5.0.2 Neutrino masses and mixings . . . . . . . . . . 102

5.0 .3 Neutrino physics in AMRB . . . . . . . . . . . . 104 
5.0 .4 Neutrino physics in PMRB . . . . . . . . . . . . 113

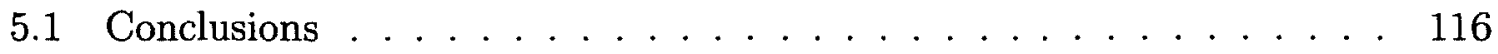

$6 U(1)_{R}$ lepton number at the LHC $\quad 118$

6.1 Sparticle decay modes . . . . . . . . . . . . . . 118

6.1.1 Neutralino and Chargino decay modes . . . . . . . . 119

6.1.2 Slepton decay modes . . . . . . . . . . . . . . 125

6.1.3 Squark decay modes . . . . . . . . . . . . 126

6.2 First and second generation squarks production . . . . . . . . . . 134

$6.2 .1 \quad \tilde{X}_{1}^{0}$ LSP topologies . . . . . . . . . . . . . 135

6.2.2 Realistic benchmark points . . . . . . . . . . . . . 140

6.3 Conclusions and Outlook . . . . . . . . . . . . . 145

7 Conclusions and Outlooks 


\section{List of Tables}

3.1 R-charge assignment for the chiral supermultiplets in the MRSSM. .

4.1 R-charge assignment for the chiral supermultiplets in our model. The subscript $a$ denote the flavour of the lepton superfield that plays the role of the down-type Higgs. The subscrit $b, c$ represent the remaining

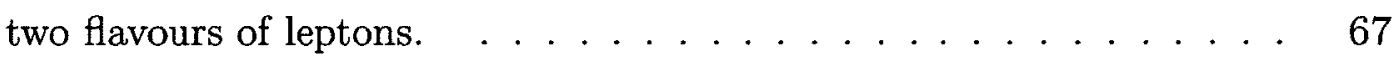

4.2 Effective vector-axial lepton couplings. . . . . . . . . . 76

4.3 R-charge assignement . . . . . . . . . . . . . . . . . . 82

4.4 R-charge assignment for the chiral supermultiplets in our model. . . . 90

4.5 The single coupling bounds on the down type Yukawa couplings, and on the interactions $\lambda$ and $\lambda^{\prime} \ldots \ldots \ldots \ldots \ldots$

5.1 Fits to neutrino oscillation data. Where two different values are present for one parameter, upper and lower row refer respectively to Normal and Inverted Hierarchy. . . . . . . . . . . . . . 102

5.2 Range of parameters used in the scan of Secs. 5.0.3-5.0.3 . . . . . . 108

6.1 The upper limit for $\sigma \times a \times \epsilon$ for all the leptonic channels, the corresponding luminosity and the ATLAS or CMS reference. . . . . . . 141

6.2 Cross section for all the possible final states for the benchmark point $\mu=-200 \mathrm{GeV}, M_{\tilde{B}}=1 \mathrm{TeV}, M_{\tilde{W}} 1.5 \mathrm{GeV}$ and $\lambda_{S}=\lambda_{T}=0.8$ and $v_{e}=10 \mathrm{GeV}$ ( in paranthesis instead the cross section is computed for $v_{e}=50 \mathrm{GeV}$. The squak masses are taken to be $700 \mathrm{GeV} . \ldots . .144$ 


\section{List of Figures}

1.1 Feynman diagram, involving a fermion anti-fermion loop, which contributes to the Higgs mass. . . . . . . . . . . . . . . 15

1.2 One loop Feynman diagram with the bosonic contribution which cancels the dangerous contribution to the scalar mass mass. . . . . . . .

2.1 b as a function of $m_{\tilde{Q}}$, for various choices of $m_{U}$ with and $f=700$ $\mathrm{GeV}$. The plot on the left is for the $S U(6)_{L} \times S U(5)_{R}$ top sector with $m_{D}=1 \mathrm{TeV}$ and the plot on the right is for the $S U(6)$ top sector. . .

2.2 Inert Higgses contribution to the $\mathrm{S}$ parameter as a function of $m_{\tilde{A}}$ and as a function of $m_{\tilde{s}}$ for various Higgs mass. . . . . . . . . . . .

2.3 T parameter contribution from the top sector as a function of $b$ for various $m_{U}$ and with $f=700 \mathrm{GeV}$. The plot on the left is for the $S U(6)_{L} \times S U(5)_{R}$ top sector with $m_{D}=1 \mathrm{TeV}$ while the plot on the right is for the $S U(6)$ top sector. . . . . . . . . . . .

2.4 Contour plots of $\Omega h^{2}$ as a function of $m_{\tilde{S}}$ and $\delta$. The region in black correspond to the dark matter abundance measured by WMAP. The plot on the left shows a low $m_{\tilde{S}}$ region, while the plot on the right shows a high $m_{\tilde{S}}$ region, both have the Standard Model Higgs mass set at $120 \mathrm{GeV} \ldots \ldots \ldots \ldots \ldots \ldots \ldots \ldots$ 
4.1 The lepton mixing with the triplet (right electron and left for the muon or for the tau) taking into account different values of the wino mass $\left(M_{\tilde{W}}=250 \mathrm{GeV}, M_{\tilde{W}}=500 \mathrm{GeV}\right.$, and $\left.M_{\tilde{W}}=1000 \mathrm{GeV}\right)$. The blue horizontal line represents the $1 \sigma$ threshold, and the one the $3 \sigma$ threshold.

4.2 The violation of leptonic universality in the charged current interaction $1-\frac{g_{\tau}}{g_{\mu}}$ assuming the the mixed lepton is the $\tau$. We considered values of $M_{\bar{W}}=250 \mathrm{GeV}, M_{\bar{W}}=500 \mathrm{GeV}$, and $M_{\tilde{W}}=1000 \mathrm{GeV}$. The horizontal line represents the $0.3 \%$ threshold, and the one the $3 \sigma$ threshold. . . . . . . . . . . . . . . . .

4.3 Majorana mass term for the neutrino $\nu_{a}$ generated at one loop from anomaly mediation ( by gaugino Majorana mass on the right, and

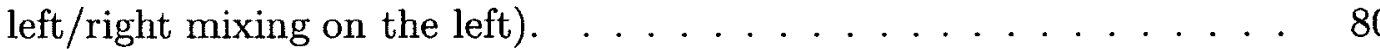

4.4 Contribution to the $\mu$ and $B_{\mu}$ terms generated by (4.54). . . . . 84

5.1 Allowed region (colored) in parameter space for the flavor assignment $A=e, B=\mu$ and $C=\tau$ in the case of Inverted Hierarchy. . . . 105

5.2 Allowed region (colored) in parameter space for the flavor assignment $A=\mu, B=e$ and $C=\tau$ in the case of Normal Hierarchy. . . . .

5.3 Allowed region (colored) in parameter space for the flavor assignment $A=\mu, B=e$ and $C=\tau$ in the case of Inverted Hierarchy. . . . 112

$6.1 \tilde{X}_{1}^{0}$ mixing angles for $M_{\tilde{W}}=1.5 \mathrm{TeV}, M_{\tilde{B}}=800 \mathrm{GeV}$ and $\mu=$ $200 \mathrm{GeV}$, exhibiting the dependence on $\lambda_{S}, \lambda_{T}$ and $v_{e}$. The plot on the left is for fixed $\lambda_{S}=\lambda_{T}=0.8$, while the one on the right is for fixed $v_{e}=10 \mathrm{GeV}$. We always plot the absolute magnitude of the rotation matrix elements, $V_{i k}^{N}$ and $U_{i k}^{N} \ldots \ldots \ldots \ldots \ldots$

$6.2 \tilde{X}_{1}^{0}$ decay modes in the case that $\tilde{X}_{1}^{0}$ is the LSP (left panel) and in the case that $\tilde{\tau}_{L}$ is the LSP (right panel). 
$6.3 \tilde{X}_{1}^{0}$ mixing angles ( left panel ) and branching ratio ( right panel ) for $\tilde{X}_{1}^{0}$ LSP scenario with for $M_{\tilde{W}}=1.5 \mathrm{TeV}, M_{\tilde{B}}=1 \mathrm{TeV}, \mu=200 \mathrm{GeV}$

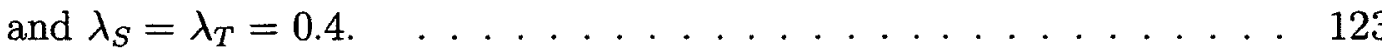

$6.4 \tilde{\tau}_{L}$ decay modes for two stau masses: $m_{\tilde{\tau}}=190 \mathrm{GeV}$ and $m_{\bar{\tau}}=$ $250 \mathrm{GeV}$. It is assumed that the lightest neutralino is heavier than the stau.

6.5 Branching fractions for the $\tilde{t}_{L}$ decay modes computed for $\lambda_{S}=\lambda_{T}=$ $0.8, \mu=200 \mathrm{GeV}, M_{\tilde{B}}=800 \mathrm{GeV}$ and $M_{\tilde{W}}=1500 \mathrm{GeV}$. We also take $\lambda_{333}^{\prime}=\left(1.8 \times 10^{-2}\right) / y_{b} \ldots \ldots \ldots \ldots \ldots \ldots \ldots$

6.6 Branching fractions for the $\tilde{b}_{L}$ decay modes computed for $\lambda_{S}=\lambda_{T}=$ $0.8, \mu=200 \mathrm{GeV}, M_{\tilde{B}}=800 \mathrm{GeV}$ and $M_{\tilde{W}}=1500 \mathrm{GeV}$. We also take $\lambda_{333}^{\prime}=\left(1.8 \times 10^{-2}\right) / y_{b}$, and add together the two neutrino channels $\left(\bar{\nu}_{e}\right.$ and $\left.\bar{\nu}_{\tau}\right) \ldots \ldots \ldots \ldots \ldots \ldots \ldots \ldots \ldots \ldots \ldots \ldots \ldots$

6.7 Branching ratios for $\tilde{t}_{R}$ decay modes computed for $\lambda_{S}=\lambda_{T}=0.8$, $\mu=200 \mathrm{GeV}, M_{\tilde{B}}=800 \mathrm{GeV}$ and $M_{\tilde{W}}=1500 \mathrm{GeV}$.

6.8 Branching ratios for $\tilde{b}_{R}$ decay modes computed for $\lambda_{S}=\lambda_{T}=0.8$, $\mu=200 \mathrm{GeV}, M_{\tilde{B}}=800 \mathrm{GeV}$ and $M_{\tilde{W}}=1500 \mathrm{GeV}$.

6.9 Left Panel:Squark Anti-Squark production cross section ( for $7 \mathrm{TeV}$ LHC run) computed for a Dirac gluino of mass ( Red) and a Majorana gluino of $M_{\tilde{g}}=2000 \mathrm{GeV}$. The $K$ factor is estimated to be $K \sim 1.6 \mathrm{In}$ the right panel we computed the ratio between the two cross sections, Dirac and Majorana. . . . . . . . . . . . . . .

6.10 Squark squark production cross section computed for a $2 \mathrm{TeV}$ Majorana and Dirac gluino. These are compared on the upper limit on the simplified models cross section for CMS searches assuming an intermediate particle of $100 \mathrm{GeV}$ and $300 \mathrm{GeV} \ldots \ldots \ldots \ldots$ 
6.11 Branching ratios for the possible LHC signals computed for $\lambda_{S}=\lambda_{T}=$ $0.8, \mu=-200 \mathrm{GeV}, M_{\tilde{B}}=1000 \mathrm{GeV}$ and $M_{\tilde{W}}=1500 \mathrm{GeV} . \ldots 142$ 6.12 Cross section for the separate production of $\tilde{X}_{1}^{0} \tilde{X}_{1}^{0}, \tilde{X}_{0}^{ \pm} \tilde{X}_{0}^{ \pm}$and $\tilde{X}_{1}^{0} \tilde{X}_{0}^{ \pm}$ through squark squark production taking into account the squarks decay modes. This is computed for $m_{\tilde{q}}=700 \mathrm{GeV}$ and $M_{\tilde{g}}=2 \mathrm{TeV}$ for a $7 \mathrm{TeV}$ LHC run. . . . . . . . . . . . . . . . . . 143 


\section{Chapter 1}

\section{The Standard Model and Beyond}

In this chapter we will introduce the Standard Model (SM), the model which describes how the different elementary particles are organized and how they interact with each other through the different forces (electromagnetic, weak and strong). The Standard Model provides a remarkably successful description of particle physics and it could potentially describe it up to very high energies since it is a well defined theory up to the Planck scale $M_{P l} \sim 10^{18} \mathrm{GeV}$. Above this scale quantum gravitational effects become important and a new framework is required. However, new physics beyond the Standard Model (BSM) should also exist below the Planck scale to explain the existence of dark matter (DM). The Standard Model describes only ordinary matter which we now know represents only the $4 \%$ of the universe. Another $23 \%$ of the universe is made of dark matter ${ }^{1}$, which doesn't emit or reflect light, but whose location and density we can estimate based on its gravitational effects on surrounding luminous matter. The Standard Model does not account for the existence of dark matter, therefore this provides the strongest evidence for BSM physics. There are also theoretical reasons which motivate the need for physics beyond the Standard Model. One of them, the hierarchy or the naturalness problem, indicates the $\mathrm{TeV}$ scale as the scale of breakdown of the Standard Model. This is the scale that the Large

\footnotetext{
${ }^{1}$ The remaining part of the universe is made by dark energy whose nature is still a mystery.
} 
Hadron Collider (LHC) at CERN is exploring. However, naturalness is a philosophical prejudice, and therefore this does not guarantee the discovery of new physics at the LHC. Historically there are many example of counter-intuitive discoveries. For example non relativistic quantum mechanics has always contradicted our common sense forcing us to accept a new ideas about nature for example with the quantum entanglement. Either way we will learn from the LHC something very interesting about nature.

\subsection{The Standard Model}

The mathematical framework of the Standard Model is quantum field theory . A quantum field theory is defined by a Lagrangian which controls the dynamics and the kinematics of the theory. Each particle is described in terms of a dynamical field that pervades the space-time. The construction of the Standard Model proceeds following the modern method of constructing most field theories: by first postulating a set of local symmetries, and then by writing down the most general Lagrangian with a given particle (field) content that respect these symmetries. The local symmetries of the SM are:

- The Poincaré group.

- The gauge group $G_{S M}=S U(3)_{c} \times S U(2)_{L} \times U(1)_{Y}$.

The matter content is defined by the quantum numbers of the various particles under the above groups and it consists of a fermionic sector ( made of quarks and leptons) and of a scalar sector which contains the Higgs boson. Matter fields are spin $1 / 2$ fermions, and depending on their gauge quantum number can divided into leptons ( neutral under $S U(3)_{c}$ ) and quarks. Lepton and quarks then come in three generations or 'flavours', with different, hierarchical, masses. The gauge group $G_{S M}$ 
describes the electromagnetic, weak and strong interactions, and invariance of the SM Lagrangian under it requires the presence of additional fields, called gauge fields, which transform as vectors under the Poincare group. The gauge fields are spin 1 bosons, namely eight gluons for the colour $S U(3)_{c}$ and four other vectors for the electroweak $S U(2)_{L} \times U(1)_{Y}$. Gauge invariance requires the gauge fields to be massless and indeed the gluons and the photon have mass zero. However, the gauge bosons $W$ and $Z^{0}$ associate to the weak force are massive, $M_{W}=80.4 \mathrm{GeV}$ and $M_{Z}=91.8$ $\mathrm{GeV}$. Also, invariance under $G_{S M}$ implies that the SM fermions should be massless as well, but we know that quarks and leptons are massive. It is here that the Higgs boson enters the picture. Both the weak gauge bosons and the SM matter fermion fields acquire mass through via the spontaneous breaking of the electroweak gauge symmetry via the Higgs boson. In this Section we will first introduce the concept of gauge symmetry and of spontaneously broken gauge symmetry. Then we will build the Lagrangian of the Standard Model.

\subsubsection{Gauge symmetry}

The Lagrangian for a free Dirac field $\psi(x)$, which describes a spin $1 / 2$ fermion, that is leptons and quarks in the $\mathrm{SM}$, is:

$$
\mathcal{L}=i \bar{\psi} \gamma^{\mu} \partial_{\mu} \psi-m \bar{\psi} \psi
$$

where we omit the dependency on the coordinate $x$. This Lagrangian is invariant under a global phase transformation

$$
\psi(x) \rightarrow \psi^{\prime}(x)=\exp [-i \alpha] \psi(x)
$$

where $\alpha$ is a real number. If one, though, requires this transformation to be local, that is

$$
\psi(x) \rightarrow \psi^{\prime}(x)=\exp [-i g \Gamma(x)] \psi(x)
$$


a new term $g \bar{\psi} \gamma^{\mu} \partial_{\mu} \Gamma \psi$ appears in the Lagrangian eq.(1.1) when the transformation is applied. In order to make the Lagrangian invariant under this local symmetry, called gauge symmetry, it is necessary to introduce a new field, $A_{\mu}(x)$, which transforms as a vector under the Poincaré group. This field is called a gauge field.

The Lagrangian for a massless free vector field is

$$
\mathcal{L}=-\frac{1}{4} F^{\mu \nu} F_{\mu \nu}
$$

where

$$
F^{\mu \nu} \equiv \partial^{\mu} A^{\nu}-\partial^{\nu} A^{\mu}
$$

Eq. 1.4 is invariant under the gauge transformation

$$
A_{\mu}(x) \rightarrow A_{\mu}^{\prime}(x)=A_{\mu}(x)+\partial_{\mu} \Gamma(x)
$$

We are allowed to add an interaction term to eq. (1.4) without spoiling its gauge invariance, and write the Lagrangian for an interacting vector field as

$$
\mathcal{L}=-\frac{1}{4} F^{\mu \nu} F_{\mu \nu}-J^{\mu} A_{\mu}
$$

where $J^{\mu}$ represents an external conserved current interacting with the system described by eq. (1.4).

The gauge-invariant Lagrangian for a theory containing both a Dirac field $\psi(x)$ and a vectorial field $A_{\mu}(x)$, therefore, is given by

$$
\mathcal{L}=i \bar{\psi} \gamma^{\mu} \partial_{\mu} \psi-m \bar{\psi} \psi-\frac{1}{4} F^{\mu \nu} F_{\mu \nu}-J^{\mu} A_{\mu}
$$

Eq. (1.8) is invariant under the gauge transformations $(1.3,1.6)$ only if we identify

$$
J^{\mu}=g \bar{\psi} \gamma^{\mu} \psi
$$

in which case we can write the Lagrangian for an interacting Dirac field $\psi(x)$ and a gauge field $A_{\mu}(x)$ as

$$
\mathcal{L}=i \bar{\psi} \gamma^{\mu} D_{\mu} \psi-m \bar{\psi} \psi-\frac{1}{4} F^{\mu \nu} F_{\mu \nu}
$$


where

$$
D_{\mu}=\partial_{\mu}+i g A_{\mu}
$$

is the covariant derivative. If one identifies $g=Q e$, where, for $\hbar=c=1, e=$ 0.3028 is the positron EM charge, eq. (1.10) is the Lagrangian describing Quantum Electrodynamics (QED).

The Lagrangian for a free complex scalar (spinless) field $\phi(x)$ is

$$
\mathcal{L}=\left(\partial_{\mu} \phi\right)^{\dagger} \partial^{\mu} \phi
$$

which is invariant under a global phase transformation of $\phi(x)$. Proceeding as we did for a Dirac field, we generalize to the case of a local phase and write

$$
\mathcal{L}=\left(D_{\mu} \phi\right)^{\dagger} D^{\mu} \phi-\frac{1}{4} F^{\mu \nu} F_{\mu \nu}
$$

which is invariant under the gauge transformation (1.6) and

$$
\phi(x) \rightarrow \phi^{\prime}(x)=\exp [-i g \Gamma(x)] \phi(x)
$$

In the present Section we have discussed the simplest example of gauge symmetry, which corresponds to an abelian $U(1)$ symmetry. The SM gauge group, as we have already mentioned, is the non abelian group $G_{S M}=S U(3)_{C} \times S U(2)_{L} \times U(1)_{Y}$. However, the generalisation is straightforward, but it requires the introduction of $n$ vector fields where $n$ is the dimension of the group associated to the gauge symmetry.

\subsection{Higgs Mechanism}

As we have mentioned in the introduction, a mass term $\left(\frac{1}{2} m^{2} A^{\mu} A_{\mu}\right)$ for a gauge field is forbidden by gauge invariance as it is easily seen from eq.(1.6). Furthermore, gauge invariance also forbids a mass terms also for the SM fermions. In the SM indeed masses are generated through spontaneous gauge symmetry breaking, and 
this requires the introduction of a scalar field in the theory, the Higgs boson. In the present Section we will illustrate the Higgs mechanism considering an abelian gauge theory.

We start by noticing that we can add to eq. (1.13) the potential:

$$
V(\phi)=m^{2} \phi^{\dagger} \phi+\frac{1}{4} \lambda\left(\phi^{\dagger} \phi\right)^{2}
$$

and that this is invariant under the gauge transformations $(1.6,1.14)$. So we write

$$
\mathcal{L}=\left(D_{\mu} \phi\right)^{\dagger} D^{\mu} \phi-V(\phi)-\frac{1}{4} F^{\mu \nu} F_{\mu \nu}
$$

If $m^{2}<0, \lambda>0$, the vacuum state, defined as the state of minimum energy of the system, belongs to the configuration $\phi(x)$ such that

$$
\left\langle 0\left|(\phi(x))^{\dagger} \phi(x)\right| 0\right\rangle=\frac{-2 m^{2}}{\lambda}=\frac{v^{2}}{2}
$$

where $v$ is the vacuum expectation value (vev).

A physical system evolves naturally to the state of minimum energy, and therefore we expand around a minimum defined by

$$
\phi(x)=\frac{1}{\sqrt{2}}(v+H(x)) \exp [i \chi(x) / v]
$$

where $H(x)$ and $\chi(x)$ are real fields. The Lagrangian in eq. (1.16) is not gauge invariant around the minimum defined by Eqs. $(1.17,1.18$ ) (the symmetry has been spontaneously broken), since $v$ is constant under gauge transformations. The field $\chi(x)$ is simply a phase which moves the system to other vacuum states. By performing a gauge transformation, we can choose a specific vacuum and set $\chi(x)$ to zero (unitary gauge). The kinetic term in eq. (1.16), re-written using eq. (1.18) and $\chi(x)=0$, reads

$$
\left(D_{\mu} \phi\right)^{\dagger} D^{\mu} \phi=\frac{1}{2} \partial_{\mu} H \partial^{\mu} H+\frac{g^{2}}{2}(v+H)^{2} A^{\mu} A_{\mu}
$$

which shows that the $A^{\mu}(x)$ has acquired a mass $m_{A}=g v$, and that $\partial^{\mu} \chi(x)$ has been replaced (or "eaten") by the longitudinal component of $A^{\mu}(x)$. Substituting eq. (1.18) 
with $\chi(x)=0$ and $m^{2}=-\frac{\lambda v^{2}}{4}$ in the potential (1.15) one obtains

$$
V(H)=\left(H^{2}+2 H v\right)\left(H^{2}+2 H v-2 v^{2}\right) \frac{\lambda}{16},
$$

from which we can see that $m_{H}=\frac{\sqrt{\lambda}}{2} v$. This process by which the gauge symmetry is spontaneously broken and consequentially a vector field acquires mass is called the "Higgs mechanism", and the massive, neutral, scalar field $H(x)$ is the Higgs boson.

\subsection{The Standard Model}

The SM's Lagrangian is invariant under $S U(3)_{c} \times S U(2)_{w} \times U(1)_{Y}$ gauge transformations, where the subscripts stand for, respectively, color, weak-isospin, and hypercharge. All the particles included in the SM have been detected. These are the quarks $u, d, c, s, t, b$, the leptons $e, \nu_{e}, \mu, \nu_{\mu}, \tau, \nu_{\tau}$, the gauge bosons $g, W^{ \pm}, Z, \gamma$, and the Higgs boson, which has been discovered at the LHC very recentely.

The quarks and the leptons are organized in three families, having the same quantum numbers but different masses. The quantum numbers of one family un$\operatorname{der} S U(3)_{c} \times S U(2)_{w} \times U(1)_{Y}$, are given by

$$
\begin{aligned}
& Q_{L}=\left(\begin{array}{c}
u_{L} \\
d_{L}
\end{array}\right) \approx\left(3,2, \frac{1}{6}\right), u_{R} \approx\left(3,1, \frac{2}{3}\right), d_{R} \approx\left(3,1,-\frac{1}{3}\right), \\
& L_{L}=\left(\begin{array}{c}
\nu_{L} \\
e_{L}
\end{array}\right) \approx\left(1,2,-\frac{1}{2}\right), e_{R} \approx(1,1,-1),
\end{aligned}
$$

where $L, R$ stand for left-handed (LH) and right-handed (RH). Here we wrote explicitly only the components relative to the $S U(2)$ transformation, otherwise the multiplet $Q_{L}$, for example, would have six components (three color eigenstates times two weak isospin eigenstates). The fermion kinetic terms are given by

$$
\mathcal{L}_{\text {fermion }}=\bar{Q}_{L} D_{\mu} \gamma^{\mu} Q_{L}+\ldots
$$


where the covariant derivative in the SM, determined by the model's gauge symmetry, is

$$
D_{\mu}=\partial_{\mu}+i c_{3} g_{s} \frac{\lambda^{a}}{2} G_{\mu}^{a}+i c_{2} g \frac{\sigma^{a}}{2} W_{\mu}^{a}+i g^{\prime} Y B_{\mu}
$$

where $\lambda^{a}$ and $\sigma^{a}$ are the Gell-Mann and Pauli matrices-respectively- which act in different subspaces, and $c_{3}, c_{2}$, and $Y$ are the gauge charges of the field on which $D_{\mu}$ acts. For an $S U(N)$ multiplet (singlet) $c_{N}=1\left(c_{N}=0\right)$. The hypercharge $Y$ is given in Eqs.(1.21).

The quantum numbers of the gauge fields are given by:

$$
G_{\mu}^{b} \approx(8,1,0), W_{\mu}^{a} \approx(1,3,0), B_{\mu} \approx(1,1,0)
$$

Their kinetic terms are given in function of the relative field strength tensor by

$$
\mathcal{L}_{\text {gauge }}=-\frac{1}{4}\left(F_{G}^{b \mu \nu} F_{G \mu \nu}^{b}+F_{W}^{a \mu \nu} F_{W \mu \nu}^{a}+F_{B}^{\mu \nu} F_{B \mu \nu}\right)
$$

The Higgs field in the SM is a doublet defined by

$$
\phi=\left(\begin{array}{c}
\phi_{+} \\
\phi_{0}
\end{array}\right) \approx\left(1,2, \frac{1}{2}\right)
$$

whose Lagrangian and potential have the usual form:

$$
\mathcal{L}_{H i g g s}=\left(D_{\mu} \phi\right)^{\dagger} D^{\mu} \phi-V(\phi), V(\phi)=m^{2} \phi^{\dagger} \phi+\frac{1}{4} \lambda\left(\phi^{\dagger} \phi\right)^{2} .
$$

If we assume that $m^{2}<0$ and $\lambda>0, \phi$ gets a non-zero vev that we choose to be real and defined by

$$
\langle 0|\phi| 0\rangle=\frac{1}{\sqrt{2}}\left(\begin{array}{c}
0 \\
v
\end{array}\right), v=2 \sqrt{\frac{-m^{2}}{\lambda}} .
$$

By convention, we chose the lower component of $\phi$ to acquire a vev. The electromagnetic charge $Q$ in the SM is then determined by

$$
Q=T^{3}+Y
$$


with $T^{3}=1 / 2(-1 / 2)$ for the upper (lower) component of an $S U(2)$ doublet and zero for a singlet.

We expand $\phi(x)$ around the ground state defined by eq. (1.28) and redefine $\phi(x)$ as

$$
\phi=\frac{1}{\sqrt{2}}\left(\begin{array}{c}
\sqrt{2} \phi_{+} \\
v+H+i \chi
\end{array}\right)
$$

Because of this shift, the electroweak symmetry $S U(2)_{w} \times U(1)$ breaks down to $U(1)_{Q}$. Once the shifted Higgs in eq.(1.30) is inserted in the Higgs Lagrangian (1.27), the covariant derivatives acting on the vev give the following gauge boson mass terms

$$
\mathcal{L}_{m a s s}=\frac{v^{2}}{8}\left(g^{2}\left(W_{\mu}^{1}-i W_{\mu}^{2}\right)\left(W_{\mu}^{1}+i W_{\mu}^{2}\right)+\left(g W_{\mu}^{3}-g^{\prime} B_{\mu}\right)^{2}\right)
$$

From this matrix we can extract the mass eigenstates (properly normalized)

$$
\begin{aligned}
& Z_{\mu}=\cos \theta_{w} W_{\mu}^{3}-\sin \theta_{w} B_{\mu} \\
& W_{\mu}^{ \pm}=\frac{1}{\sqrt{2}}\left(W_{\mu}^{1} \mp i W_{\mu}^{2}\right), \tan \theta_{w}=\frac{g^{\prime}}{g}
\end{aligned}
$$

with the respective eigenvalues

$$
m_{Z}^{2}=\frac{1}{4}\left(g^{\prime 2}+g^{2}\right) v^{2}, m_{W^{ \pm}}^{2}=\frac{1}{4} g^{2} v^{2}
$$

The massless eigenstate is identified with the photon $A_{\mu}$ :

$$
A_{\mu}=\cos \theta_{w} B_{\mu}+\sin \theta_{w} W_{\mu}^{3}
$$

Its coupling is therefore defined equal to $e$ and given by

$$
e=\frac{g^{\prime} g}{\sqrt{g^{\prime 2}+g^{2}}} .
$$

A Dirac mass term like the one written in eq. (1.10) would explicitly break the $S U(2)_{w}$ gauge symmetry, since

$$
\psi_{L / R}=\frac{1}{2}\left(1 \mp \gamma^{5}\right) \psi, \quad m \bar{\psi} \psi=m \bar{\psi}_{L} \psi_{R}+h . c .
$$


The masses of the fermions are a consequence of EWSB as well, through the Yukawa interaction terms:

$$
\mathcal{L}_{Y u k a w a}=-\bar{Q}_{L} y_{u} \phi u_{R}-\bar{Q}_{L} y_{d} \tilde{\phi} d_{R}-\bar{L}_{L} y_{e} \phi e_{R}+h . c .
$$

where $y_{u, d, e}$ are $3 \times 3$ matrices in family space ${ }^{2}$ and $\tilde{\phi}=i \sigma_{2} \cdot \phi^{*}$. In case of a single family the fermion masses would turn out to be

$$
m_{u}=y_{u} \frac{v}{\sqrt{2}}, m_{d}=y_{d} \frac{v}{\sqrt{2}}, m_{e}=y_{e} \frac{v}{\sqrt{2}}, m_{\nu}=0
$$

The complete Lagrangian of the SM is then:

$$
\mathcal{L}_{S M}=\mathcal{L}_{\text {fermion }}+\mathcal{L}_{\text {gauge }}+\mathcal{L}_{\text {Higgs }}+\mathcal{L}_{\text {Yukawa }}
$$

and we see that it contains operators of dimension 4 or smaller, i.e. it is a renormalisable quantum field theory. This means that it is a well defined theory up to very high energies, $M_{\text {Planck }} \sim 10^{18} \mathrm{GeV}$. At energies above the Planck scale gravity becomes important and shoud be included in the framework, but this is a problem which goes beyond our interests. However, as we have mentioned in the introduction to this chapter, there are reasons both experimental and theoretical which call for BSM physics at lower energies. In the next Section we will briefly introduce them.

\subsection{Physics Beyond the Standard model}

In this Section, we will first briefly summarise the experimental and theoretical motivations for physics beyond the Standard Model, and then we will focus on the hierarchy problem which is the theoretical motivation for physics beyond the standard model at the $\mathrm{TeV}$ scale.

\footnotetext{
${ }^{2}$ in eq. (1.37) we suppressed the family indices.
} 


\subsubsection{Dark matter}

The most solid reason for BSM physics is the existence of dark matter ${ }^{3}$ which has been confirmed from various observations of galaxy clusters, the galactic rotation curves, and cosmological data. So we know now that $23 \%$ of the universe is made by matter completely neutral under the electromagnetic interaction. This calls for the existence of at least one new electrically-neutral and stable particle to play the role of dark matter. The Standard Model does not provide a dark matter candidate since neutrinos, which are the only electromagnetic neutral SM particle, cannot provide all the dark matter present in the universe. It is then necessary to introduce a new particle to account for the whole dark matter present in the universe. However we do not know what are the spin, mass or interactions of this particle, and in fact we do not know whether there is a single particle or a complicated hidden sector including various kinds of particles.

\subsubsection{Theoretical motivation for BSM physics}

Here is a list of the main theoretical reasons for BSM physics:

- The strong CP problem: it is the question of why QCD does not lead to large CP violation. The non observation of a neutron electric dipole moment implies that the operator $\epsilon_{\mu \nu \lambda \tau} G^{\mu \nu} G^{\lambda \tau}$, with $G_{\mu \nu}$ being the gluon field strength, has a coefficient smaller than $10^{-12}$. The SM does not provide any reasons why this coefficient is so small.

- Baryogenesis: the asymmetry between matter and antimatter in the observable universe calls for a mechanism, baryogenesis, to explain it.The SM does not provide such a mechanism.

\footnotetext{
${ }^{3}$ Strictly speaking also the observation of neutrino oscillation is an evidence of BSM physics. However, it is possible to easily accomodate neutrino masses and mixings in the Standard Model
} 
- The pattern of fermion masses and mixings does not have a rationale in the SM.

- Gauge couplings unification: we find experimentally that $g_{s}=1.12, g=0.65$ and $g^{\prime}=0.35$ at $E \sim M_{Z}$. It would be interesting to understand the reason for such difference.

- Number of families: matter is made of three families, why?

- Hierarchy problem, which is the traditional theoretical argument for physics beyond the Standard Model at the Tev scale. In a Quantum Field Theory the squared mass parameter $m_{h}^{2}$ of a scalar particle receives quantum corrections growing like $\Lambda^{2}$ where $\Lambda$ is the UV cutoff of the theory. If we insist in having a very large cut-off (i.e. the Planck scale), we must expect large scalar masses, while now we know that $m_{h} \sim 125 \mathrm{GeV}$ - In other words the SM does not provide an explanation of why $m_{h}<<M_{P l}$. This is the Hierarchy problem.

In the following Section we will explain more in the detail what the hierarchy problem is and we will discuss some of its possible solutions.

\subsection{The Hierarchy problem and its solutions}

\subsubsection{The Hierarchy problem}

The Higgs boson is a spin 0 particle that, as we just learnt, has a mass of $\sim 125$ $\mathrm{GeV}$. It is the only scalar of the SM and for this reason it is also a source of one of the most severe theoretical problems of the SM, the Hierarchy problem. Indeed in quantum field theory the mass of a scalar particle is very sensitive to the mass of heavy states to which the scalar couples directly or indirectly. Indeed at one loop the scalar mass receive corrections proportional to $\Lambda^{2} /\left(16 \pi^{2}\right)$ where $\Lambda$ is the cutoff 
of the theory. Therefore, it is very unnatural to expect a hierarchy $m_{h}<<\Lambda$ since this requires to tune the parameter $m$ for the scalar mass in the Lagrangian with a precision of 32 digits to produce the right order of magnitude for $m_{h} \sim 125 \mathrm{GeV}$ and $\Lambda \sim M_{P l}$. This is the reason why the hierarchy problem is also called naturalness or fine tuning problem.

The Standard Model with a cut-off near the maximum attainable energy at the Tevatron is natural, and we should not be surprised that we have not observed any new physics. However, the Standard Model with a cut-off of order the LHC energy would be fine tuned, and so we should expect to see new physics at the LHC. In this Section we will introduce two classes of model which solve the hierarchy problem: supersymmetric models and little higgs models as they are the subject of the present thesis.

\subsubsection{Little Higgs models}

Little Higgs models [4] are weakly coupled extensions of the Standard Model with little or no fine-tuning, describing physics up to an energy scale of around $10 \mathrm{TeV}$. In these models the Standard Model Higgs boson is a pseudo-Goldstone boson of a spontaneously broken approximate global symmetry and is kept light by a mechanism known as collective symmetry breaking. Collective symmetry breaking means that the global symmetry is broken only if more than one coupling is turned on, namely the Higgs is massless if we set any one of these couplings to zero. Thanks to this mechanism all one loop quadratic divergences (which involve one coupling alone) to the Higgs mass are avoided.

The spectrum of new particles varies somewhat from one Little Higgs model to another, but all of them predict at least one vector-like quark at the $\mathrm{TeV}$ scale, along with extra gauge bosons and scalars. The original Little Higgs models were plagued by strong constraints from electroweak precision data which required a fine-tuning to 
be reintroduced. An economical solution to this problem is to introduce a discrete symmetry called T-parity. T-parity not only eliminates most constraints from electroweak precision data, but it also leads to a promising dark matter candidate, as for example the heavy photon $A_{H}$.

\subsubsection{Supersymmetry}

Sensitivity of the mass to the heavy states is a feature only of scalar fields. For fermions and gauge bosons, the loop corrections are instead proportional to their mass, and therefore quantum field theories containing light fermions and gauge bosons are perfectly natural. The reason is that fermions masses are protected by chiral symmetries and gauge bosons by the gauge symmetries. The fundamental idea behind supersymmetry is to provide a symmetry which also protects scalar masses. This new symmetry relates scalars to fermions, namely to each boson supersymmetry associates a fermionic partner, called a superpartner, and vice-versa. In this ways the dangerous loop corrections to scalar masses at the origin of the hierarchy problem are cancelled by the supersymmetric partners of the particle running in the loop. We will illustrate this cancellation by considering the one loop contribution to the Higgs mass of a SM fermions $f$ which couple to the Higgs through the Yukawa couplings eq. 1.37. The diagram is shown in Fig. (1.1) and gives a contribution

$$
\Delta_{f} m_{H}^{2}=-\frac{\left|y_{f}^{2}\right|}{8 \pi^{2}} \Lambda^{2}
$$

where $\Lambda$ is the SM cutoff and $y_{f}$ is the $f$ Yukawa coupling. As already explained, since this contribution is quadratic in the cutoff, taking $\Lambda \sim M_{P l}$ requires a huge fine-tuning, one part in $10^{17}$, of the parameter $m^{2}$ in the Lagrangian eq.(1.27) to maintain the Higgs mass at the electroweak scale. In supersymmetric extensions of the SM, which we will describe in the third chapter of the thesis, to each SM fermion correspond a scalar partner. This gives another one loop contribution to the Higgs 


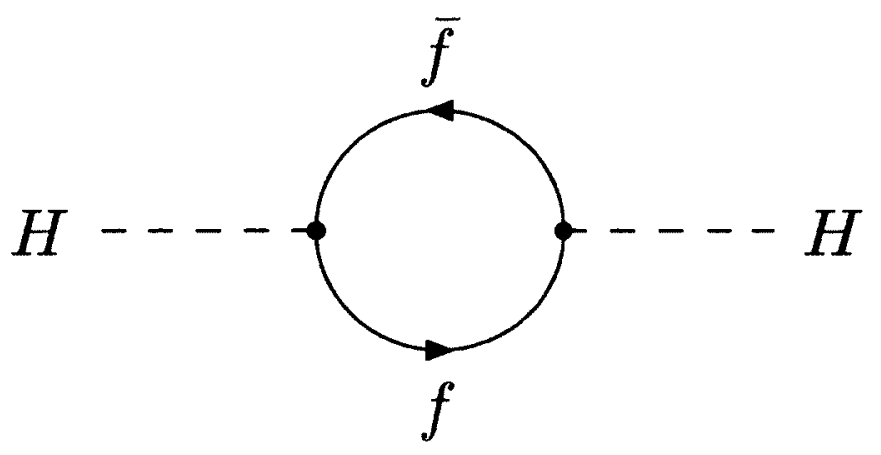

Figure 1.1: Feynman diagram, involving a fermion anti-fermion loop, which contributes to the Higgs mass.

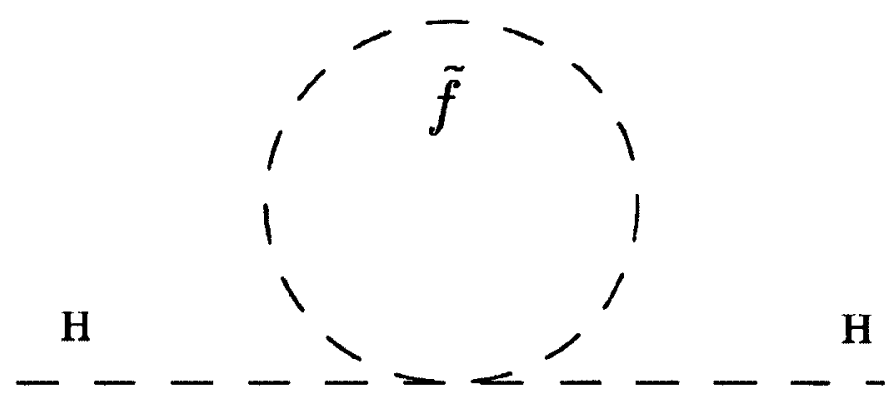

Figure 1.2: One loop Feynman diagram with the bosonic contribution which cancels the dangerous contribution to the scalar mass mass.

mass, shown Fig. 1.2, which cancel the dangerous contribution in eq. (1.40). This is in a nutshell how supersymmetry solves the hierarchy problem. 


\section{Chapter 2}

\section{UV friendly $\mathbf{T}$ parity in the $S U(6) / S p(6)$ Little Higgs model}

\subsection{Little Higgs models, $T$ parity and strongly cou- pled UV completion}

As we have explained in the previous chapter, Little Higgs models are weakly coupled extensions of the Standard Model with little or no fine-tuning, describing physics up to an energy scale of around $10 \mathrm{TeV}$. Little Higgs models are constructions where the electroweak symmetry is broken by a scalar Higgs field which is light because it is a pseudo-Goldstone boson $[4-8]$ (see $[9,10]$ for an older realization of the Higgs as a pseudo-Goldstone). To realize this idea, first a global symmetry group is spontaneously broken via an unspecified mechanism. This will generate various Goldstone bosons, among which the Higgs. Then weak couplings are introduced to give the Higgs appropriate gauge, Yukawa and scalar quartic couplings. In order for these couplings not to introduce large radiative corrections to the Higgs mass, they must break the global symmetry only collectively [7]. That is each coupling alone, while breaking a part of the global group, must leave enough of it unbroken such that the Higgs remains an exact massless Goldstone boson. Only when two or more weak 
couplings are introduced in the theory will the Higgs get a mass, guaranting that it will remain parametrically light. In this way, the Higgs mass can be made lighter than $f$, the Goldstone boson decay constant, by a loop factor and therefore the cutoff of the theory where the Goldstone bosons become strongly coupled can be as high as $\sim 4 \pi f \sim 10 \mathrm{TeV}$.

The description of the Higgs and its couplings at low energy, i.e. below $\sim 10 \mathrm{TeV}$, can be done using non-linear sigma model fields parametrized by a set of pseudoGoldstone bosons. Besides the usual Standard Model fields, this description will include extra scalars, extra gauge bosons and extra colored fermions. These new states have masses near $f \sim 1 \mathrm{TeV}$ and are responsible for cancelling the one loop quadratic divergences to the Higgs mass that occur in the Standard Model. Above $10 \mathrm{TeV}$, the derivative couplings of the Goldstone bosons render the theory strongly coupled and a new description, a ultraviolet (UV) completion, has to be used.

The main phenomenological problems of Little Higgs models come from the fact that the new $\mathrm{TeV}$ states can contribute to electroweak precision observables at tree level [12-19]. These contributions are parametrically of the same size has loop contributions of Standard Model particles or contributions from a strongly coupled sector at the $\mathrm{TeV}$ scale such as technicolor. These tree-level contributions are calculable, and can be made small but in general they limit the parameter space of Little Higgs models significantly. A perhaps more elegant solution is to invoke a symmetry principle to forbid the dangerous tree level contributions. The most economical symmetry would be a $Z_{2}$ symmetry, similar to the $R$-parity that is imposed in the MSSSM, under which all the new states are odd while the Standard Model particles are even. In the context of Little Higgs, such a parity was defined in various models and dubbed T-parity [20-22].

In the Littlest Higgs model [7] for example the T-parity acts schematically as [21]:

$$
\Sigma \rightarrow \Omega \Sigma^{\dagger} \Omega^{\dagger}
$$


where $\Sigma=\exp (2 i \pi) \Sigma_{0}$ is a $5 \times 5$ non-linear sigma model field containing the Goldstone bosons that transforms as a symmetric two-index tensor of the global $S U(5)$ and $\Omega$ is an element of $S O(5)$. It was however later realized that this type of parity might not be straightforward to implement in a UV completion $[23,24]$. For example in a strongly coupled UV completion where the non-linear sigma model field is a composite of fermions $\psi_{a}[25]$ (with $a$ a $S U(5)$ index):

$$
\Sigma_{a b} \sim \psi_{a} \psi_{b}
$$

T-parity in the UV theory would translate to:

$$
\psi_{a} \rightarrow \psi_{b}^{\dagger}
$$

which is not a symmetry of the fermionic kinetic terms. Only in combination with a space-time parity: $\mathbf{x} \rightarrow-\mathbf{x}$, is this a symmetry. The manifestation of this fact in the low energy theory is the presence of WZW-like terms that break $T$-parity $[23,24]$ (but not the T-parity combine with $\mathbf{x} \rightarrow-\mathbf{x}$ ). While those terms do not lead to dangerous contributions to electroweak precision measurements, they make the dark matter candidate unstable. And while one could imposing such a space-time parity on the gauge sector, the situation is more problematic in the fermion sector. Again schematically, T-parity invariant Yukawa couplings require terms of the form:

$$
Q_{1} \Sigma u_{1}^{c}+Q_{2} \Sigma^{\dagger} u_{2}^{c}
$$

where the two terms are related by T-parity. In a UV completion, these terms could come, for example from 4-Fermi operators:

$$
\frac{Q_{1}^{a} \psi_{a} \psi_{b} u_{1}^{c b}+Q_{2}^{a} \psi_{a}^{\dagger} \psi_{b}^{\dagger} u_{2}^{c b}}{f^{2}}
$$

where, again the two terms need to have the same coefficient. In this case it is hard to see what symmetry of the UV theory could relate the two terms ${ }^{1}$.

\footnotetext{
${ }^{1}$ In fact, since the Standard Model is not symmetric under $\mathbf{x} \rightarrow-\mathbf{x}$, this symmetry would need to be broken somehow.
} 
In the following sections we examine the possibilities for redefining T-parity so that it could be integrated without difficulty in a UV completion. We find the $S U(6) / S p(6)$ Little Higgs model [26] particularly amenable to such a redefinition ${ }^{2}$. After exposing the leading order gauge and fermionic structure of the a T-symmetric version of the $S U(6) / S p(6)$ model in sections 2.2 , we compute various radiative corrections to the Higgs potential in section 2.3. We finally discuss the consequences of the model for electroweak precision tests in section 2.5 and for dark matter abundance and phenomenology in sections 2.6 and 2.7 .

\subsubsection{T-parity in the Littlest Higgs model}

Before turning to the $S U(6) / S p(6)$ model we now discuss briefly the challenges associated with building a strongly coupled UV completion to the Littlest Higgs model with T-parity. To implement T-parity in a Littlest Higgs-like model with an $S U(5) / S O(5)$ global group structure, in a way that does not involve the complex conjugate of the non-linear sigma model field as in (2.1), one could imagine using instead an exchange symmetry [31]. The field content could be doubled, with two non-linear sigma model fields $\Sigma_{A}$ and $\Sigma_{B}$ which are interchanged under T-parity. The Lagrangian for the non-linear sigma model fields would simply be:

$$
\mathcal{L}=\left|D_{\mu} \Sigma_{A}\right|^{2}+\left|D_{\mu} \Sigma_{B}\right|^{2}
$$

where both transform as $\Sigma_{i} \rightarrow U \Sigma_{i} U^{T}$ under $S U(5)$. The field content is doubled, and contain both odd and even fields. To decouple the unwanted field, we would need to write a term of the form:

$$
c \Lambda^{2} f^{2} \operatorname{Tr}\left(\Sigma_{A} \Sigma_{B}^{\dagger}\right)
$$

In the limit where $c \sim 1$, the even combination of the Goldstone fields decouple and one is left with the original Littlest Higgs model. The main complication for the UV

\footnotetext{
${ }^{2}$ This is also one of the two smallest special coset models without a dangerous singlet $[27,28]$
} 
completion is then to generate that term. For example, let's imagine that the fields $\Sigma_{A}$ and $\Sigma_{B}$ are composite of strongly interacting fermions $\psi_{A}$ and $\psi_{B}$ respectively. Both $\psi_{A}$ and $\psi_{B}$ are charged under a different strongly interacting $S O(N)$ gauge group and are are fundamental of an $S U(5)$ flavor group. In fact there are now also two different $S U(5)$ global flavor groups, one associated to $\psi_{A}$, the other to $\psi_{B}$. To break this enlarge flavor group to the diagonal through a term of the form of (2.7), one could write 4 -fermi operator:

$$
\operatorname{Tr}\left(\frac{\psi_{A} \psi_{A}^{T} \psi_{B}^{*} \psi_{B}^{\dagger}}{M^{2}}\right)
$$

This would require the introduction of a new scale $M$, close to $f$. Note also that (2.8) needs to be $S U(5)$ invariant. Generating (2.7) with a coefficient proportional to $f^{4}$ instead of $f^{2} \Lambda^{2}$ might be easier as the $S U(5)$ invariance requirement would be softened, and the mass scale $M$, could be above $\Lambda$, in a regime where $\psi_{A}$ and $\psi_{B}$ are still weakly coupled. In this case however, the new even fields have masses of order $f \sim 1 \mathrm{TeV}$, and one must check that they do not reintroduce large correction to the electroweak precision observables. This could lead to a viable model but the structure of the theory, if not the low energy theory at least the UV completion would need to be enlarged. Note however that the situation is simpler in a weakly coupled supersymmetric UV completion such as the one built in [33].

\subsection{The SU(6)/Sp(6) model with T-parity}

\subsubsection{Global symmetries}

We now turn to the $S U(6) / S p(6)$ model [26], for which we find an alternative to doubling the content of the theory. A successful implementation of a T-parity needs to lead to new gauge bosons that are odd, andnnew scalars that are odd, especially 
if these new scalars contain a triplet and finally, in the fermion sector we need the Standard Model fermions to be even.

In the Littlest Higgs the gauge sector consists of two $S U(2)$ groups: $S U(2)_{A}$ and $S U(2)_{B}$, and possibly two $U(1)$ groups. The Standard Model gauge bosons and the new $\mathrm{TeV}$ scale gauge bosons are orthogonal linear combinations of $V_{A}^{\mu}$ and $V_{B}^{\mu}$, the gauge bosons associated with the two groups. To make the Standard Model gauge bosons even and the new $\mathrm{TeV}$ scale gauge boson odd, we need a $Z_{2}$ symmetry that would interchange $V_{A}^{\mu}$ and $V_{B}^{\mu}$. In a prototype UV completion with strongly coupled fermions (see for example [25]), this would mean:

$$
\psi_{2_{A}} \rightarrow \psi_{2_{B}}
$$

where $\psi_{2_{A}}$ and $\psi_{2_{B}}$ are doublet of $S U(2)_{A}$ and $S U(2)_{B}$ respectively. However, in a Littlest Higgs type model, $\psi_{2_{A}}$ and $\psi_{2_{B}}$ have different hypercharge, so this $Z_{2}$ symmetry would not commute with $U(1)_{Y}$. If we consider instead the $S U(6) / S p(6)$ model, where the equivalent fields have hypercharge 0 , this sort of symmetry can be implemented. The spontaneous breaking of the global $S U(6)$ to $S p(6)$ is in this case parametrized at low energy by the $6 \times 6$ non-linear sigma model field:

$$
\Sigma=e^{i \Pi_{a} X_{a} / f} \Sigma_{0} e^{i \Pi_{a} X_{a}^{T} / f}=e^{2 i \Pi_{a} X_{a} / f} \Sigma_{0}
$$

where

$$
\Sigma_{0}=f\left(\begin{array}{ccc}
0 & 0 & -\mathbb{1}_{2 \times 2} \\
0 & \epsilon_{2 \times 2} & 0 \\
\mathbb{1}_{2 \times 2} & 0 & 0
\end{array}\right) \text {, }
$$

is the vacuum of the theory before electroweak symmetry breaking and each entry in the matrix is itself a $2 \times 2$ matrix with $\epsilon$ the $2 \times 2$ antisymmetric matrix with 
$\epsilon_{12}=-1$. There are 14 Goldstone bosons, which can be written in matrix form:

$$
\Pi=\left(\begin{array}{cccc}
\tilde{\phi}-\frac{\eta}{2} & h_{A} & h_{B} & \chi \epsilon \\
h_{A}^{\dagger} & \frac{\eta}{2} & 0 & -h_{B}^{T} \\
h_{B}^{\dagger} & 0 & \frac{\eta}{2} & h_{A}^{T} \\
-\epsilon \chi^{\dagger} & -h_{B}^{*} & h_{A}^{*} & \tilde{\phi}^{T}-\frac{\eta}{2}
\end{array}\right) .
$$

where $\tilde{\phi}$ is an hermitian $2 \times 2$ matrix, $h_{A}$ and $h_{B}$ are 2 components column vectors, $\eta$ is a real field and $\chi$ a complex field. Gauge quantum numbers of these fields will be determined by the gauging of various subgroups of the global $S U(6)$ group presented in the next subsection.

\subsubsection{Gauge interactions}

Gauge interactions are introduced as usual by gauging two $S U(2)$ subgroups of the $S U(6)$ global group:

$$
\begin{aligned}
& X_{A}^{a}=\left(\begin{array}{ccc}
\sigma^{a} & 0 & 0 \\
0 & 0_{2 \times 2} & 0 \\
0 & 0 & 0_{2 \times 2}
\end{array}\right), \\
& X_{B}^{a}=\left(\begin{array}{ccc}
0_{2 \times 2} & 0 & 0 \\
0 & 0_{2 \times 2} & 0 \\
0 & 0 & -\sigma^{a *}
\end{array}\right),
\end{aligned}
$$

where $\sigma_{a}$ are the Pauli matrices with $\operatorname{Tr}\left(\sigma_{a} \sigma_{b}\right)=\delta_{a b} / 2$. Only the diagonal subgroup, identified with the Standard Model $S U(2)_{L}$ is left unbroken by the vacuum, leaving one massless $S U(2)$ triplet of gauge bosons, and one massive one. The gauge couplings 
associated with $S U(2)_{A}$ and with $S U(2)_{B}$ will be equal due to T-parity: $g_{A}=g_{B}=g$. We can also establish that $\phi$ is a triplet of $S U(2)_{L}$, while $h_{A}$ and $h_{B}$ are doublets and $\chi$ and $\eta$ are singlets. The hypercharge gauge couplings are introduce by gauging a different subgroup of $S p(6)$ :

$$
Y=\left(\begin{array}{cccc}
0_{2 \times 2} & 0 & 0 & 0 \\
0 & 1 / 2 & 0 & 0 \\
0 & 0 & -1 / 2 & 0 \\
0 & 0 & 0 & 0_{2 \times 2}
\end{array}\right)
$$

with the hypercharge gauge boson.

Implementing T-parity in the fermion sector, which will be presented in the next section, is made easier by the introduction of a third $S U(2)$. This can be seen in the following way: if we introduce a fermion doublet $q_{A}$ transforming under $S U(2)_{A}$, T-parity forces the introduction of another doublet transforming under $S U(2)_{B}$ :

$$
q_{A} \rightarrow q_{B}
$$

The odd combination must be made massive, and an even combination will be massless and identified with a SM fermion doublet. Naively, this can be done by adding a mass term of the form

$$
\left(q_{A}-q_{B}\right) q_{C}^{c}
$$

This is invariant under the Standard Model $S U(2)$ but not invariant separately under $S U(2)_{A}$ and $S U(2)_{B}$. To make it invariant, we add a third $S U(2)$ gauge group called $S U(2)_{C}$, whose associated gauge boson is even under T-parity. We break the resulting $S U(2)^{3}$ gauge group to its diagonal subgroup by adding two link fields $K_{A}$ and $K_{B}$ $([33,34])$ that transform as bifundamental of $S U(2)_{A} \times S U(2)_{C}$ and $S U(2)_{B} \times S U(2)_{C}$ 
respectively and get vev's proportional to the identity:

$$
\begin{array}{ll}
K_{A} \rightarrow U_{A} K_{A} U_{C}^{\dagger}, & \left\langle K_{A}\right\rangle=\mathbb{1}_{2 \times 2} \\
K_{B} \rightarrow U_{B} K_{B} U_{C}^{\dagger}, & \left\langle K_{B}\right\rangle=\mathbb{1}_{2 \times 2}
\end{array}
$$

where $U_{A}, U_{B}$ and $U_{C}$ are elements of $S U(2)_{A}, S U(2)_{B}$ and $S U(2)_{C}$ respectively. The Lagrangian for the kinetic term of the Goldstone bosons of the theory is given by:

$$
\mathcal{L}_{\text {gauge }}=\frac{1}{8} f^{2} \operatorname{Tr}\left|D_{\mu} \Sigma\right|^{2}+f_{k}^{2} \operatorname{Tr}\left|D_{\mu} K_{A}\right|^{2}+f_{k}^{2} \operatorname{Tr}\left|D_{\mu} K_{B}\right|^{2}
$$

where $K_{A}$ and $K_{B}$ can be written in term of the Goldstone modes $\phi_{A}^{a}$ and $\phi_{B}^{a}$ as:

$$
K_{A}=\exp \left(i \phi_{A}^{a} \frac{\sigma_{a}}{f_{k}}\right) \quad K_{B}=\exp \left(i \phi_{B}^{a} \frac{\sigma_{a}}{f_{k}}\right)
$$

The gauge bosons mass eigenstates and their masses can be derived from equation $(2.20)$ :

$$
\begin{gathered}
M_{\tilde{V}}^{2}=\frac{1}{2} g^{2}\left(f^{2}+2 f_{k}^{2}\right), \quad \tilde{V}^{\mu}=\frac{\left(V_{A}^{\mu}-V_{B}^{\mu}\right)}{\sqrt{2}}, \\
M_{V_{H}}^{2}=f_{k}^{2}\left(g+2 g_{C}^{2}\right), \quad V_{H}^{\mu}=\frac{\left(g V_{A}^{\mu}+g V_{B}^{\mu}-2 g_{C} V_{C}^{\mu}\right)}{\sqrt{2 g^{2}+4 g_{C}^{2}}}, \\
M_{V_{S M}}^{2}=0, V_{S M}^{\mu}=\frac{\left(g_{C} V_{A}^{\mu}+g_{C} V_{B}^{\mu}+g V_{C}^{\mu}\right)}{\sqrt{g^{2}+2 g_{C}^{2}}} .
\end{gathered}
$$

Two of the gauge bosons, $V_{\mathrm{SM}}^{\mu}$ and $V_{H}^{\mu}$, are even and one, $\tilde{V}^{\mu}$, is odd under T-parity, which acts on the gauge bosons as

$$
V_{A}^{\mu} \rightarrow V_{B}^{\mu}, \quad V_{C}^{\mu} \rightarrow V_{C}^{\mu}
$$

Note that $A_{H}$ is even, but can be made heavy (with mass of order $\Lambda$ ) by taking $g_{C}$ large. The Standard Model $S U(2)_{L}$ is the diagonal subgroup of the $3 S U(2)$ 's and its gauge coupling is given

$$
\frac{1}{g_{\mathrm{SM}}^{2}}=\frac{1}{g_{A}^{2}}+\frac{1}{g_{B}^{2}}+\frac{1}{g_{C}^{2}}=\frac{2}{g^{2}}+\frac{1}{g_{C}^{2}}
$$


In the limit where $g_{C} \gg 1$, we have :

$$
g_{\mathrm{SM}}=\frac{g}{\sqrt{2}}
$$

To find the action of T-parity on the field $\Sigma$ it is useful to think of it as a composite of strongly interacting fermions:

$$
\Sigma \sim \Psi \Psi^{T}
$$

where $\Psi$ form a 6 of the $S U(6)$ flavor symmetry. The quantum numbers of the different components of $\Psi$ are as follow:

\begin{tabular}{|l||l||l||l||}
\hline Field & $S U(2)_{1}$ & $S U(2)_{2}$ & $U(1)_{Y}$ \\
\hline$\psi_{2_{A}}$ & 2 & 1 & 0 \\
\hline$\psi_{0}$ & 1 & 1 & $\frac{1}{2}$ \\
\hline$\psi_{0}^{\prime}$ & 1 & 1 & $-\frac{1}{2}$ \\
\hline$\psi_{2_{B}}$ & 1 & 2 & 0 \\
\hline
\end{tabular}

with two $S U(2)$ doublets and two singlets, for a total of six fields. The T-parity action is then:

$$
\psi_{2_{A}} \rightarrow \epsilon \psi_{2_{B}} \quad \psi_{0} \rightarrow \psi_{0} \quad \psi_{0}^{\prime} \rightarrow \psi_{0}^{\prime} \quad \psi_{2_{B}} \rightarrow-\epsilon \psi_{2_{A}}
$$

This leads to the following transformation for the non-linear sigma model field at low energy:

$$
\Sigma \rightarrow T \Sigma T^{T}
$$

where $T$ is an element of $S p(6)$ and is given by:

$$
T=\left(\begin{array}{ccc}
0 & 0 & \epsilon \\
0 & \mathbb{1} & 0 \\
-\epsilon & 0 & 0
\end{array}\right)
$$


This in turn leads to the following action on the components of the $\Pi$ field:

$$
\begin{gathered}
\eta \rightarrow \eta, \\
\tilde{\phi} \rightarrow-\tilde{\phi}, \\
\chi \rightarrow-\chi^{*}, \\
h_{A} \rightarrow-\epsilon h_{B}^{*} .
\end{gathered}
$$

T-even and T-odd Higgs doublets can then be defined:

$$
\begin{aligned}
H & =\frac{h_{B}+\epsilon h_{A}^{*}}{\sqrt{2}}, \\
\tilde{H} & =\frac{h_{B}-\epsilon h_{A}^{*}}{\sqrt{2}}
\end{aligned}
$$

where here and in the rest of the paper, the fields with a tilde are odd under T-parity. The T-parity action on the Higgs fields will lead to an inert doublet Higgs model [35]

as will discuss in more details later. The $S U(2)$ triplets $\tilde{\phi}, \phi_{A}$ and $\phi_{B}$ form two T-odd and one T-even combination. Two of them, one even and one odd, are eaten by the massive gauge boson $V_{H}^{\mu}$ and $\tilde{V}^{\mu}$, while one of the odd combination:

$$
\tilde{\Phi}=\frac{\phi_{A}-\phi_{B}-\tilde{\phi}}{\sqrt{3}}
$$

will be part of the low energy specturm.

\subsubsection{Top sector}

As in most Little Higgs models, the third generation of quarks is treated differently than the other fermions as only the top Yukawa is large enough to introduce a finetuning problem in a model with a UV cutoff at $10 \mathrm{TeV}$. As such it is the only one that needs to be introduced in a way that respect the collective symmetry breaking mechanism. There is some freedom on how to do that as one can choose to keep a very minimal particle content that respect only a small subset of the global symmetries, or 
opt for a more symmetric coupling between the fermions and non-linear sigma model field. In the most symmetric case, $Q_{3}$ and $Q_{3}^{c}$, two six-plet of fermions are introduced and coupled to $\Sigma$ in an $S U(6)$ invariant way. This creates many massive vector-like fermions and massless combinations can be recovered by adding $q_{C}$, a $S U(2)_{C}$ doublet and a singlet $u^{\prime c}$. The Lagrangian is given by:

$$
\mathcal{L}_{\text {top }}^{S U(6)}=f\left[\lambda_{1} Q_{3}{ }^{T} \Sigma^{\dagger} Q_{3}^{c}+\lambda_{2}\left(-q_{C}^{T} K_{1}^{T} \epsilon q_{A}^{c}+q_{C}^{T} K_{2}^{T} q_{B}^{c}\right)+\lambda_{3} u \tilde{u}^{c}\right]+\text { h.c. }
$$

where $q_{A}^{c}, q_{B}^{c}$ and $q_{C}$ form $S U(2)$ doublets and $Q_{3}$ and $Q_{3}^{c}$ are sixplet of the global $S U(6)$ :

$$
Q_{3}=\left(\begin{array}{c}
q_{A} \\
u \\
d \\
q_{B}
\end{array}\right), \quad Q_{3}^{c}=\left(\begin{array}{c}
q_{A}^{c} \\
d^{c} \\
u^{c} \\
q_{B}^{c}
\end{array}\right)
$$

Under T-parity we have:

$$
Q_{3} \rightarrow T Q_{3} \quad Q_{3}^{c} \rightarrow T Q_{3}^{c} \quad \tilde{u}^{c} \rightarrow \tilde{u}^{c} \quad q_{C} \rightarrow q_{C}
$$

Replacing $\Sigma, K_{A}$ and $K_{B}$ by their vev (but setting the Higgses vev to 0 ), we get:

$$
\mathcal{L}_{\text {top }}^{S U(6)}=\lambda_{1} f\left(q q^{c}+\tilde{q} \tilde{q}^{c}+d d^{c}\right)+\frac{\lambda_{2}}{\sqrt{2}} f q_{C} q^{c}+f u\left(\lambda_{3} \tilde{u}^{c}-\lambda_{1} u^{c}\right)
$$

where:

$$
\begin{array}{ll}
q=\frac{q_{A}+\epsilon q_{B}}{\sqrt{2}} & q^{c}=\frac{q_{B}^{c}-\epsilon q_{A}^{c}}{\sqrt{2}} \\
\tilde{q}=\frac{q_{A}-\epsilon q_{B}}{\sqrt{2}} & \tilde{q}^{c}=\frac{-q_{B}^{c}-\epsilon q_{A}^{c}}{\sqrt{2}}
\end{array}
$$

So we have two massive ( with mass of order $f$ ) vector-like doublets and two massive vector-like $\mathrm{T}$-even singlets. The remaining $\mathrm{T}$-even doublet and singlet are massless 
at this point. They are to first approximation the Standard Model top quark doublet and singlet, and get a mass after electroweak symmetry breaking. The masses of the heavy fields are:

$$
\begin{array}{ll}
m_{Q}=\sqrt{\lambda_{1}^{2}+\lambda_{2}^{2}} f, & m_{\bar{Q}}=\lambda_{1} f, \\
m_{U}=\sqrt{\lambda_{1}^{2}+\lambda_{3}^{2}} f, & m_{D}=\lambda_{1} f
\end{array}
$$

and the mass eigenstates are given by:

$$
\begin{aligned}
q_{\mathrm{SM}} & =\frac{\lambda_{2} q-\lambda_{1} q_{C}}{\sqrt{\lambda_{1}^{2}+\lambda_{2}^{2}}}=\sin \left(\theta_{D}\right) q-\cos \left(\theta_{D}\right) q_{C} \\
u_{\mathrm{SM}}^{c} & =\frac{\lambda_{1} \tilde{u}^{c}+\lambda_{3} u^{c}}{\sqrt{\lambda_{1}^{2}+\lambda_{3}^{2}}}=\cos \left(\theta_{S}\right) u^{\prime c}+\sin \left(\theta_{S}\right) u^{c} \\
Q & =\cos \left(\theta_{D}\right) q+\sin \left(\theta_{D}\right) q_{C} \\
U^{c} & =\sin \left(\theta_{S}\right) u^{\prime c}-\cos \left(\theta_{S}\right) u^{c}
\end{aligned}
$$

From these mass eigenstates we can read off the top Yukawa coupling which is given by:

$$
\lambda_{t}=\frac{\sqrt{2} \lambda_{1} \lambda_{2} \lambda_{3}}{\sqrt{\lambda_{1}^{2}+\lambda_{2}^{2}} \sqrt{\lambda_{1}^{2}+\lambda_{3}^{2}}}=\frac{\sin 2 \theta_{D} \sin \theta_{S} m_{Q}}{\sqrt{2} f} .
$$

Note that this implies a lower bound on $m_{Q}$ :

$$
m_{Q}>\sqrt{2} \lambda_{t} f
$$

The main issue with this top sector is that it contains the T-even $d^{c}$ state which can lead to large contribution to $Z \rightarrow b \bar{b}$ when exchanged at tree-level [14]. There are various possibility to remedy this problem. For example, $d^{c}$ can be removed from 
$Q_{3}^{c}$. The top sector Lagrangian then becomes:

$$
\mathcal{L}_{\text {top }}^{S U(6)_{L} \times S U(5)_{R}}=\lambda_{1} f\left(\begin{array}{c}
q_{A}^{c} \\
q_{A} \\
u
\end{array}\right) \Sigma_{B}^{\dagger}\left(\begin{array}{c}
0 \\
0 \\
u^{c} \\
q_{B}^{c}
\end{array}\right)+f \frac{\lambda_{2}}{\sqrt{2}}\left(-q_{C}^{T} K_{A}^{T} \epsilon q_{A}^{c}+q_{C}^{T} K_{B}^{T} q_{B}^{c}\right)+\lambda_{3} f u \tilde{u}^{c}+\lambda_{4} f d
$$

This Lagrangian contains a additional free parameter $\lambda_{4}$, but the dangerous coupling of $d^{c}$ to the Higgs is removed. The masses of the various states are still given by (2.39) except for $m_{D^{c}}$ which becomes:

$$
m_{D}=\lambda_{4} f
$$

The top Yukawa remains is also still given by equation (2.41).

Another possibility is to remove the down-like states completely as in the $S U(5)_{M L S}$ top sector of [14]. Being less symmetric, the $S U(5)_{M L S}$ and $S U(6)_{L} \times$ $S U(5)_{R}$ top sectors lead to larger deviation to the $T$ parameter as we will see in section 2.5. Also, the radiative corrections to the Higgs potential coming from the $S U(6)$ top sector is one-loop finite and calculable. In the $S U(6)_{L} \times S U(5)_{R}$ top sector, only the Peccei-Quinn violating $b^{2}$ term is finite and calculable, while in the $S U(5)_{M L S}$ top sector, both the masses and $b^{2}$ term are log-divergent at one-loop and therefore not calculable.

\subsubsection{Light Fermions}

While the top quark Yukawa coupling needs to be introduced in a way that respect collective symmetry breaking, this is not true for the other fermions. They can arise from two doublets $q_{A} \in 2_{S U(2)_{A}}$ and $q_{B} \in 2_{S U(2)_{B}}$ which can be embedded in a $S U(6)$ 
incomplete multiplet:

$$
Q_{1,2}=\left(\begin{array}{c}
q_{A} \\
0 \\
0 \\
q_{B}
\end{array}\right)
$$

on which the $\mathrm{T}$ parity acts as:

$$
Q_{1,2} \rightarrow T Q_{1,2}
$$

which means $q_{A} \rightarrow \epsilon q_{B}$. The $\mathrm{T}$ parity eigenstates are the odd linear combination: $\tilde{q}=\frac{q_{A}-\epsilon q_{B}}{\sqrt{2}}$ and the even one: $q_{\mathrm{SM}}=\frac{q_{A}+\epsilon q_{B}}{\sqrt{2}}$ which is identified with the SM doublet. In order to give mass to the odd doublet we introduce a $S U(2)_{C}$ doublet $\tilde{q}_{C}^{c}$ odd under T parity $\tilde{q}_{C}^{c} \rightarrow-\tilde{q}_{C}^{c}$ which can marry the $\tilde{q}$ with the help of the link fields:

$$
\frac{k}{\sqrt{2}} f_{k}\left[q_{A}^{T} K_{A}^{*}+q_{B}^{T} \epsilon K_{B}^{*}\right] \tilde{q}_{C}^{c}
$$

which gives mass to the odd Dirac pair:

$$
m_{\tilde{q}}=k f_{k}
$$

leaving the $\mathrm{T}$ even linear combination massless.

The Yukawa interactions for the light fermions can be written down by introducing the SM singlets simply as T-even fields embed in incomplete $S U(6)$ multiplets :

$$
\mathcal{U}_{1,2}^{c}=\left(\begin{array}{c}
0 \\
u^{c} \\
0 \\
0
\end{array}\right), \quad \mathcal{D}_{1,2}^{c}=\left(\begin{array}{c}
0 \\
0 \\
d^{c} \\
0
\end{array}\right) .
$$


The up and down type Yukawa coupling can then be written as:

$$
k_{u} f Q_{1,2}^{T} \Sigma^{\dagger} \mathcal{U}_{1,2}^{c}+k_{d} f Q_{1,2}^{T} \Sigma^{\dagger} \mathcal{D}_{1,2}^{c}
$$

and the full Lagrangian for the light standard model fermions becomes:

$$
\mathcal{L}_{\text {light }}=k_{u} f Q_{1,2}^{T} \Sigma^{\dagger} \mathcal{U}_{1,2}^{c}+k_{d} f Q_{1,2}^{T} \Sigma^{\dagger} \mathcal{D}^{c}+\frac{k}{\sqrt{2}} f_{k}\left[q_{A}^{T} K_{A}^{*}+q_{B}^{T} \epsilon K_{B}^{*}\right] \tilde{q}_{C}^{c}+\text { h.c. }
$$

We note that the fermion content presented here contains $S U(2)_{i}^{2} U(1)_{Y}$ anomalies (with $i=A, B, C$ ), however it is straightforward to add heavy fields, with mass of order $\sim 10 \mathrm{TeV}$, to cancel them.

\subsection{Radiative corrections}

The gauge and Yukawa interactions introduced in the previous section will radiatively induce additional contributions to the Lagrangian of the theory. The form and size of the new terms depend on the way the interactions involved in the radiative corrections break the various global symmetries of the theory. For example, turning on only one gauge coupling, or only one top Yukawa coupling preserve enough of global symmetry so that the Higgs remains an exact Goldstone boson and contributions proportional to, for example, $g^{2}$ cannot include a Higgs mass term. At one loop, the radiative corrections can be quadratically divergent, log-divergent or finite. In the case of quadratic or log divergence, this indicates that the correction is sensitive in the UV and cannot be fully computed in the low energy theory. The corresponding operator should be included in the Lagrangian with an unknown coefficient. However the size of the one-loop quadratic or log-divergent diagram constitute an natural estimate for the coefficient of this operator ${ }^{3}$. In the case where terms in the effective Lagrangian

\footnotetext{
${ }^{3}$ more precisely, a spurion analysis has to be performed, to make sure that, for example that the size of the one-loop log-divergent operator is not parametrically smaller than the size of an operator generated via a two-loops quadratically divergent operator.
} 
only appear via finite one-loop diagram, this means that they are dominated by IR physics and are therefore computable. There might be divergent contributions to the same terms at higher loop order, but these will be subdominant. We now examine the size of the principal radiative corrections.

\subsubsection{One-loop quadratic divergence}

At one loop, gauge interactions will generate a quartic coupling for the Higgses which can be found by computing the Coleman-Weinberg potential [36]:

$$
V_{g^{2}}=\frac{g^{2}}{16 \pi^{2}} f^{2} \Lambda^{2}\left[c_{1} \operatorname{Tr}\left(\Sigma Q_{A}^{a} \Sigma^{\dagger} Q_{A}^{a}\right)+c_{2} \operatorname{Tr}\left(\Sigma Q_{B}^{a} \Sigma^{\dagger} Q_{B}^{a}\right)\right]
$$

where $c_{1}$ and $c_{2}$ are of order 1 and $T$-parity guarantees $c_{1}=c_{2}=c^{4}$ Those two operators yield a mass of order $f \sim 1 \mathrm{TeV}$ for the singlet $\chi$ and an order one quartic couplings for the two Higgs doublets:

$$
V_{g^{2}}=c f^{2}\left|\chi-\frac{h_{A}^{T} \epsilon h_{B}}{f}\right|^{2}+c f^{2}\left|\chi+\frac{h_{A}^{T} \epsilon h_{B}}{f}\right|^{2}=c\left[f^{2}|\chi|^{2}+\left|h_{A}^{T} \epsilon h_{B}\right|^{2}\right]
$$

The other operator to receive a 1-loop quadratically divergent contribution comes from the hypercharge sector:

$$
V_{g^{\prime 2}}=c_{Y} \frac{3 g^{\prime 2}}{16 \pi^{2}} f^{2} \Lambda^{2} \operatorname{Tr}\left(\Sigma Y \Sigma^{\dagger} Y\right)=c_{Y} f^{2} \Lambda^{2} \frac{3 g^{\prime 2}}{16 \pi^{2}}\left[\left(\frac{\left|h_{A}\right|^{2}+\left|h_{B}\right|^{2}}{f^{2}}\right)+\frac{2}{3}\left(\frac{\left|h_{A}\right|^{2}+\left|h_{B}\right|^{2}}{f^{2}}\right)^{2}\right]+\cdots
$$

with $c_{Y} \sim 1$. Since a collective breaking mechanism was not implemented in this sector, it contains a mass term for the Higgses. Being quadratically divergent, the size of this operator is not calculable, nevertheless, taking $c_{Y} \sim 1$ will lead to an appropriately small Higgs mass, owing to the smallness of the $U(1)_{Y}$ gauge coupling. In fact, with a Higgs mass of $120 \mathrm{GeV}$ and a cutoff of $\Lambda=10 \mathrm{TeV}$, the fine-tuning due to the hypercharge one loop quadratic divergence is about 1 part in 4 (see [35]).

\footnotetext{
${ }^{4}$ The Coleman-Weinberg potential computation actually yield a negative value for $c$ which if correct would signal that we are in the wrong vacuum [30]. However, since the coefficient of those operators are not calculable we assume that they are positive and that we are in the correct vacuum.
} 


\subsubsection{One-loop log divergence}

A few operators will be generated with coefficient proportional to $g_{A}^{2} g_{B}^{2}=g^{4}$. Being proportional to two weak coupling constants they have coefficients parametrically smaller than (2.54), but they provide one of the dominant contribution to the Higgs mass. For example, the one loop log-divergent contribution to Higgs potential is given by:

$V_{g^{4}}\left(h_{A}, h_{B}\right)=\frac{9 g_{A}^{2} g_{B}^{2}}{16 \pi^{2}}\left[f^{2}\left(\left|h_{A}\right|^{2}+\left|h_{B}\right|^{2}\right)-\left(\frac{5}{6}\left(\left|h_{A}\right|^{2}+\left|h_{B}\right|^{2}\right)^{2}-\frac{2}{3}\left|h_{A}^{T} \epsilon h_{B}\right|^{2}\right)\right] \log \frac{\Lambda^{2}}{M_{\tilde{V}}^{2}}$.

Note that the Higgs quartic couplings generated in (2.53), (2.54) and (2.55) all respect an extra symmetry that guarantees that the charged Higgs and CP-even neutral Higgs will have the same mass. The $S U(2)^{\prime} s$ gauge couplings leave unbroken a custodial $S U(2)_{\text {custodial }}$ under which the $2 \times 2$ matrix $\mathcal{H}$ with $h_{A}$ and $h_{B}$ as columns transforms as:

$$
\mathcal{H} \rightarrow U \mathcal{H} U^{\dagger} \text { with } \quad U \in S U(2)_{\text {custodial }}
$$

Together with a Pecci-Quinn symmetry, this guarantees that the operators generated only through $S U(2)_{1}$ and $S U(2)_{2}$ gauge couplings will produce quartic couplings of the form:

$$
c_{1}\left(\left|h_{A}\right|^{2}+\left|h_{B}\right|^{2}\right)^{2}+c_{3}\left|h_{A}^{T} \epsilon h_{B}\right|^{2}
$$

On the other hand, the hypercharge gauge coupling leaves an $S p(4)$ symmetry invariant (acting on indices 1,2,5 and 6 of the $\Sigma$ field) which forces the Higgs quartic interaction to be of the form

$$
\left(\left|h_{A}\right|^{2}+\left|h_{B}\right|^{2}\right)^{2}
$$

The leading quartic interaction that will induce a difference in mass difference between the charged and CP-even, T-odd Higgses come from operators generated with coefficient proportional to $g^{2} g^{\prime 2}$. At one loop there is a log divergent contribution 
given by:

$$
V_{g^{2} g^{\prime 2}}\left(h_{A}, h_{B}\right)=-\frac{3 g^{2} g^{\prime 2}}{16 \pi^{2}}\left[\left(\left|h_{A}\right|^{2}-\left|h_{B}\right|^{2}\right)^{2}+4\left|h_{A}^{T} \epsilon h_{B}\right|^{2}\right] \log \frac{\Lambda^{2}}{m_{\tilde{V}}^{2}} .
$$

\subsubsection{One-loop finite correction}

As mentioned above, the one-loop log divergent and quadratically divergent contributions to the potential are not calculable. They are sensitive to the UV completion and they correspond to operators that have to be included in the Lagrangian with unknown coefficients. For example, in addition to 1-loop log-divergent contribution proportional to $g_{A}^{2} g_{B}^{2}$, there might also be two loop quadratically divergent contributions with the same size.

There are also terms which get their main contribution from one loop finite term and are therefore calculable. The odd triplet $\tilde{\Phi}$ for example gets a calculable mass given by:

$$
M_{\tilde{\Phi}}^{2}=\frac{6}{(4 \pi)^{2}} \frac{f^{2} f_{k}^{2} g_{A}^{2} g_{B}^{2} g_{C}^{2}}{\left(M_{V_{H}}^{2}-M_{\tilde{V}}^{2}\right)} \log \frac{M_{V_{H}}}{M_{\bar{V}}}
$$

Note however that as $g_{C}$ approaches $4 \pi$ and $V_{H}$ becomes heavy, pertubativity in $g_{C}$ is lost, and while we expect the size of this mass to remain of the same order, it will not be calculable anymore.

The $S U(6)$ top sector has extra spurious symmetries and its dominant contribution to the Higgs potential is a one loop finite piece. This sector also breaks the PQ symmetry, so the quadratic term will also contain a ' $b^{2}$ term': $h_{A}^{T} \epsilon h_{B}$. It is in fact the dominant contribution to the $b^{2}$ term which is therefore calculable:

$$
V_{\mathrm{top}}\left(h_{A}, h_{B}\right)=-\frac{3}{8 \pi^{2}} \lambda_{t}^{2} \frac{m_{Q}^{2} m_{U}^{2}}{m_{Q}^{2}-m_{U}^{2}} \log \left(\frac{m_{Q}^{2}}{m_{U}^{2}}\right)\left[\left|h_{A}\right|^{2}+\left|h_{B}\right|^{2}-\left(h_{A}^{T} \epsilon h_{B}+\text { h.c. }\right)\right]
$$

For the $S U(6)_{L} \times S U(5)_{R}$ top sector, with $d^{c}$ removed from $Q_{3}^{c}$, the 1-loop contribution to $\left|h_{A}\right|^{2}+\left|h_{B}\right|^{2}$ is log-divergent, but the coefficient of the $b^{2}$ term remains 


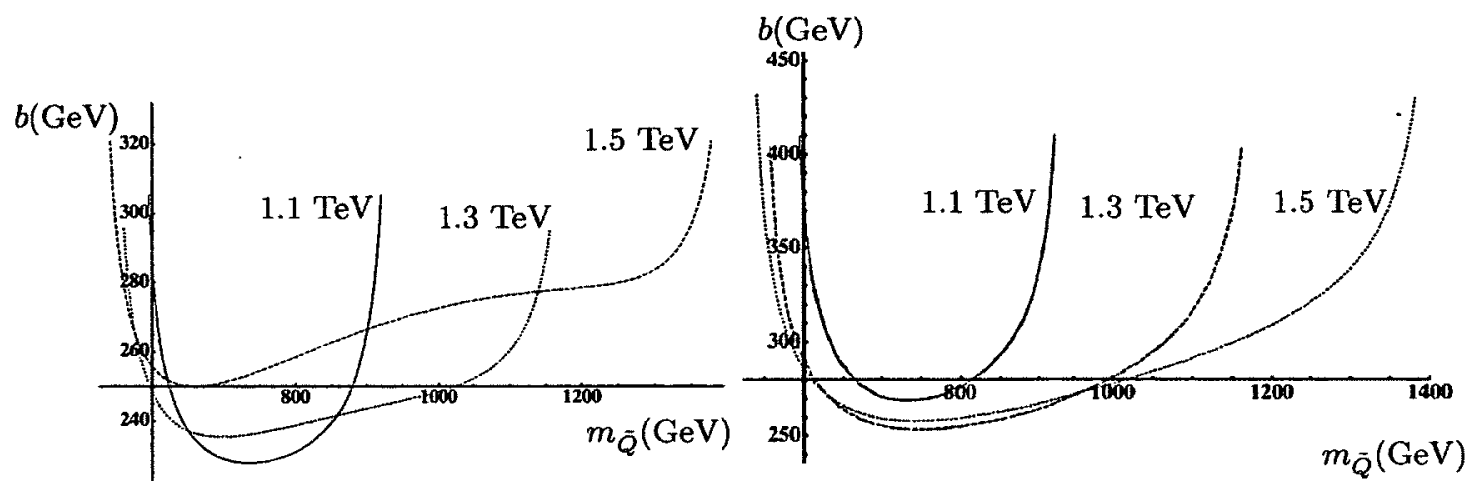

Figure 2.1: $\mathrm{b}$ as a function of $m_{\tilde{Q}}$, for various choices of $m_{U}$ with and $f=700 \mathrm{GeV}$. The plot on the left is for the $S U(6)_{L} \times S U(5)_{R}$ top sector with $m_{D}=1 \mathrm{TeV}$ and the plot on the right is for the $S U(6)$ top sector.

finite and calculable. It is given by:

$b^{2}=-\frac{3}{4 \pi^{2}}\left[\lambda_{1}^{2}\left(\frac{m_{Q}^{2} m_{D}^{2}}{m_{Q}^{2}-m_{D}^{2}} \log \frac{m_{D}^{2}}{m_{Q}^{2}}+\frac{m_{\tilde{Q}}^{2} m_{D}^{2}}{m_{\tilde{Q}}^{2}-m_{D}^{2}} \log \frac{m_{\tilde{Q}}^{2}}{m_{D}^{2}}\right)+\lambda_{t}^{2} \frac{m_{Q}^{2} m_{U}^{2}}{2\left(m_{Q}^{2}-m_{U}^{2}\right)} \log \frac{m_{U}^{2}}{m_{Q}^{2}}\right]$

The value of $b^{2}$ as a function of $m_{\tilde{Q}}$ is shown in figure 2.1 for $f=700 \mathrm{GeV}$ and various choices of quark masses and for both top sectors.

We note that the singlet $\eta$ does not get any mass from radiative corrections. It can be given a small mass explicitely [14].

\subsection{Electroweak symmetry breaking}

We now examine the Higgs potential in more details. Because of the T-parity, the Higgs potential will be of the inert doublet type $[35,38]$. In the $h_{A}$ and $h_{B}$ basis, the most general potential that respect T-parity is given by:

$$
\begin{aligned}
V\left(h_{A}, h_{B}\right)= & m^{2}\left(\left|h_{A}\right|^{2}+\left|h_{B}\right|^{2}\right)+b^{2}\left(h_{A}^{T} \epsilon h_{B}+\text { h.c. }\right) \\
& +c_{1}\left(\left|h_{A}\right|^{4}+\left|h_{B}\right|^{4}\right)+c_{2}\left|h_{A}\right|^{2}\left|h_{B}\right|^{2}+c_{3}\left|h_{A}^{T} \epsilon h_{B}\right|^{2}+ \\
& \quad \frac{c_{4}}{2}\left[\left(h_{A} h_{B}\right)^{2}+\text { h.c. }\right]+\frac{c_{5}}{2}\left[\left(h_{A} h_{B}\right)\left(\left|h_{A}\right|^{2}+\left|h_{B}\right|^{2}\right)+\text { h.c }\right]
\end{aligned}
$$


In our model, we expect $c_{3}$ to be the dominant coupling as it receives an order 1 contribution from the quadratic divergence of equation (2.53). The coefficients $c_{1}$ and $c_{2}$ also receive contribution from a quadratic divergence from the hypercharge sector (see equation (2.54)) but due to the smallness of $g^{\prime}$ we expect them to be somewhat smaller than $c_{3}$. Contributions to $c_{2}-2 c_{1}$ are of order 1-loop and come from log-divergent contributions such as the one given in equation (2.59) or possibly from the top sector:

$$
c_{2}-2 c_{1} \equiv \delta \sim \frac{1}{16 \pi^{2}} .
$$

The Peccei-Quinn violating quartic $c_{4}$ and $c_{5}$ are also only generated at one loop.

To minimize the potential and find the mass eigenstates, it is useful to write it in term of the T-parity eigenstate $H, \tilde{H}$ (see (2.32)):

$$
\begin{aligned}
& V(H, \tilde{H})=\mu_{+}^{2}|H|^{2}+\mu_{-}^{2}|\tilde{H}|^{2} \\
& \quad+\lambda_{1}|H|^{4}+\lambda_{2}|\tilde{H}|^{4}+\lambda_{3}|H|^{2}|\tilde{H}|^{2}+\lambda_{4}\left|H^{\dagger} \tilde{H}\right|^{2}+\frac{\lambda_{5}}{2}\left[\left(H^{\dagger} \tilde{H}\right)^{2}+\text { h.c. }\right]
\end{aligned}
$$

with

$$
\begin{aligned}
& \mu_{+}^{2}=m^{2}-b^{2} \\
& \mu_{-}^{2}=m^{2}+b^{2} \\
& \lambda_{1}=\frac{c_{1}}{2}+\frac{c_{2}}{4}+\frac{c_{3}}{4}+\frac{c_{4}}{4}+\frac{c_{5}}{2} \\
& \lambda_{2}=\frac{c_{1}}{2}+\frac{c_{2}}{4}+\frac{c_{3}}{4}+\frac{c_{4}}{4}-\frac{c_{5}}{2} \\
& \lambda_{3}=c_{1}+\frac{c_{2}}{2}-\frac{c_{3}}{2}-\frac{c_{4}}{2} \\
& \lambda_{4}=c_{1}-\frac{c_{2}}{2}+\frac{c_{3}}{2}-\frac{c_{4}}{2} \\
& \lambda_{5}=c_{1}-\frac{c_{2}}{2}-\frac{c_{3}}{2}+\frac{c_{4}}{2}
\end{aligned}
$$


This potential has an extremum where only $H$ gets a vev and T-parity is unbroken:

$$
\langle H\rangle=\left(\begin{array}{c}
0 \\
\frac{v}{\sqrt{2}}
\end{array}\right) \quad\langle\tilde{H}\rangle=\left(\begin{array}{l}
0 \\
0
\end{array}\right) .
$$

At this point $H$ is identical to a Standard Model Higgs doublet, with 3 of its components eaten by the $W$ and $Z$ boson and the remaining scalar $h$ being the usual neutral Higgs boson. The T-odd doublet $\tilde{H}$ contains a charged scalar $\tilde{h}^{+}$, a neutral CP odd scalar $\tilde{A}$ and a neutral CP even scalar $\tilde{S}$. The masses for these fields are given by [39]:

$$
\begin{aligned}
m_{h}^{2} & =-2 \mu_{+}^{2}=2 \lambda_{1} v^{2}=2\left(b^{2}-m^{2}\right) \\
m_{\tilde{h}^{+}}^{2} & =\mu_{-}^{2}+\lambda_{3} \frac{v^{2}}{2} \\
m_{\tilde{S}}^{2} & =\mu_{-}^{2}+\left(\lambda_{3}+\lambda_{4}+\lambda_{5}\right) \frac{v^{2}}{2} \\
m_{\tilde{A}}^{2} & =\mu_{-}^{2}+\left(\lambda_{3}+\lambda_{4}-\lambda_{5}\right) \frac{v^{2}}{2}
\end{aligned}
$$

As long as the parameters are such that these masses are positive, this vacuum will be a minimum of the potential. With only the dominant quartic coupling $c_{3}$ non-zero we have: $\lambda_{1}=\lambda_{2}=-\lambda_{3} / 2=\lambda_{4} / 2=-\lambda_{5} / 2$ which implies the following relationships among the masses:

$$
\begin{aligned}
m_{\tilde{h}^{+}}^{2} & =m_{S}^{2} \\
m_{\tilde{A}}^{2} & =m_{\tilde{S}}^{2}+m_{h}^{2}
\end{aligned}
$$

In term of the parameters in (2.63) we have $m_{\tilde{S}^{2}}^{2}=m_{\tilde{h}^{+}}^{2}=2 m^{2}$ and $m_{\tilde{A}}^{2}=2 b^{2}$, where the second relation always hold if the Peccei-Quinn violating couplings $c_{4}$ and $c_{5}$ are 0 . Since $m_{h}>0$ requires $b>m$, we find that $\tilde{A}$, the $\mathrm{T}$ and CP odd scalar is the heaviest among the Higgs states. The presence of a non-zero $c_{1}$ and $c_{2}$ will cause $m_{\tilde{h}^{+}}^{2}$ and $m_{\tilde{S}}^{2}$ to deviate from $2 m^{2}$ while a deviation from the relation $c_{2}=2 c_{1}$ will lead to 
$m_{\tilde{h}^{+}} \neq m_{\tilde{S}^{*}}$

$$
m_{\tilde{h}^{+}}^{2}=m_{\tilde{S}^{+}}^{2}+\frac{\delta}{2} v^{2}
$$

Since we want $\tilde{S}$ to be the dark matter candidate, we require $m_{\tilde{h}^{+}}>m_{S}$ which in turn require $2 c_{1}>c_{2}$. Finally we also note that we expect the Higgs mass $m_{h}$ to be of the same order of $b$, unless there is some fine tuning between $m$ and $b$, as seen from equation (2.68).

\subsection{Electroweak Precision Observables}

\subsubsection{Higgs sector contributions}

Because of the T-parity, there are no tree level contributions to electroweak precision observables. There are however 1-loop contributions to the $S$ and $T$ parameters [41]. The largest contributions will come from 'light' fields with mass of order $f /(4 \pi)$ while contributions from loops of 'heavy' field, with mass of order $f$ are parametrically suppressed, but can give numerically large contributions in the case of the top sector. The 'light' fields that could contribute are the odd triplet $\tilde{\Phi}$ and the odd Higgses. The first gives a small contribution because it has a small coupling to the SM Higgs. In the case of loops of the odd Higgses, $\tilde{S}, \tilde{h}^{+}$and $\tilde{A}$, the contribution to $T$ is small due to the approximate custodial symmetry of the Higgs potential (which leads to the degeneracy in $\tilde{S}$ and $\tilde{h}^{+}$).

The Higgs doublets also give a contribution to the $\mathrm{S}$ parameter [42]:

$$
S=\frac{1}{\pi m_{Z}^{2}}\left[B_{22}\left(m_{Z}^{2}, m_{\tilde{S}}^{2}, m_{\tilde{A}}^{2}\right)-B_{22}\left(m_{Z}^{2}, m_{\tilde{S}}^{2}, m_{\tilde{A}}^{2}\right)\right]
$$


where:

$$
\begin{gathered}
B_{22}\left(q^{2}, m_{1}^{2}, m_{2}^{2}\right)=b_{22}\left(q^{2}, m_{2}^{2}, m_{2}^{2}\right)-b_{22}\left(0, m_{1}^{2}, m_{2}^{2}\right), \\
b_{22}\left(q^{2}, m_{2}^{2}, m_{1}^{2}\right)=\frac{1}{4}(\Delta+1)\left[m_{1}^{2}+m_{2}^{2}-\frac{1}{3} q^{2}\right]-\frac{1}{2} \int_{0}^{1} d x X \log (X-i \epsilon), \\
X=m_{1}^{2} x+m_{2}^{2}(1-x)-q^{2} x(1-x),
\end{gathered}
$$

and $\Delta$ is the regulator of dimensional regularization defined by:

$$
\Delta=\frac{2}{4-n}+\log (4 \pi)+\gamma
$$

$n$ is the number of space-time dimensions and $\gamma$ is the Euler's constant. The terms proportional to $\Delta$ must clearly exactly cancel in the computation of the physical observables. In figure 2.2 we show the contribution of the Higgs sector to the $S$ parameter for various Standard Model Higgs masses when the relationships (2.72) and (2.73) hold.

\subsubsection{Fermionic sector contributions}

Loops of top partners, even if their contributions are parametrically suppressed can lead to numerically significant contributions to the $T$ parameter [14]. In figure 2.3 we show the contribution to $T$ for the $S U(6)$ and $S U(6)_{L} \times S U(5)_{R}$ top sectors for $f=700 \mathrm{GeV}$ and $M_{Q}=1 \mathrm{TeV}$, as a function of $b$. We see that the contributions from the $S U(6)$ top sector are smaller due to the fact that this the top Yukawa coupling was implemented in a way that respects a custodial symmetry.

The light fermions will not contribution to the oblique parameter as their coupling to the Higgs sector is very small. On the other hand the Lagrangian eq.(2.51) contains vertices with one standard model fermion, the odd partner and the triplet $\tilde{\Phi}$ :

$$
k f_{k} q_{\mathrm{SM}}^{\dagger} \tilde{\Phi} \tilde{q}
$$



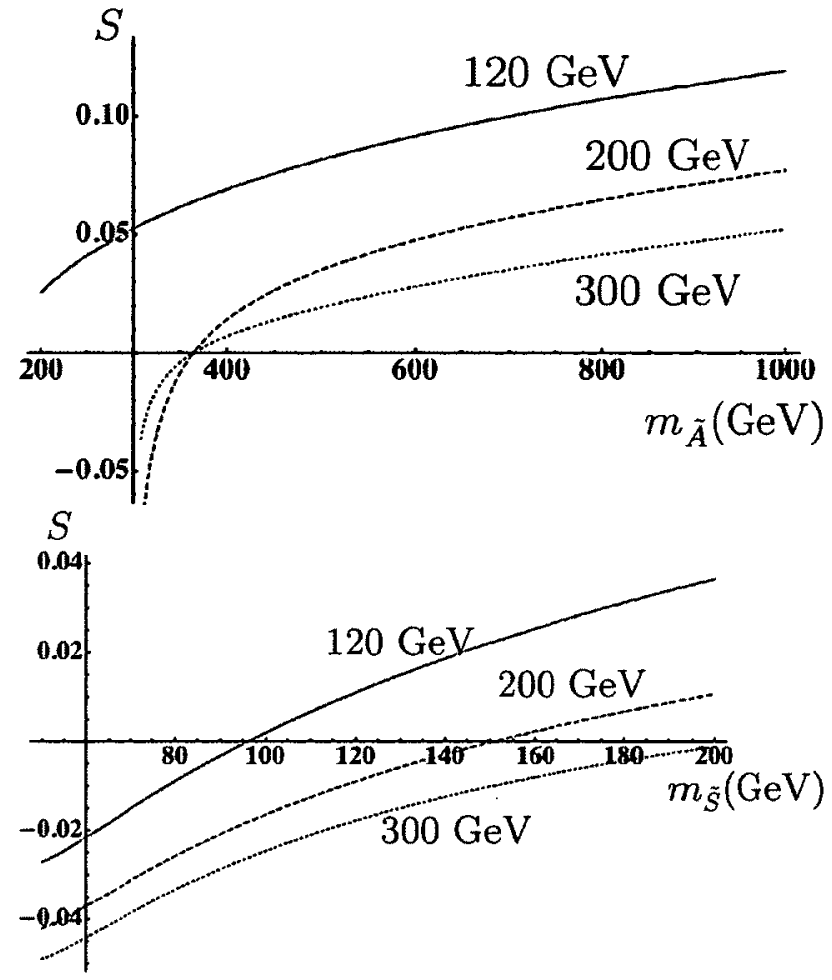

Figure 2.2: Inert Higgses contribution to the $S$ parameter as a function of $m_{\tilde{A}}$ and as a function of $m_{\tilde{S}}$ for various Higgs mass. 

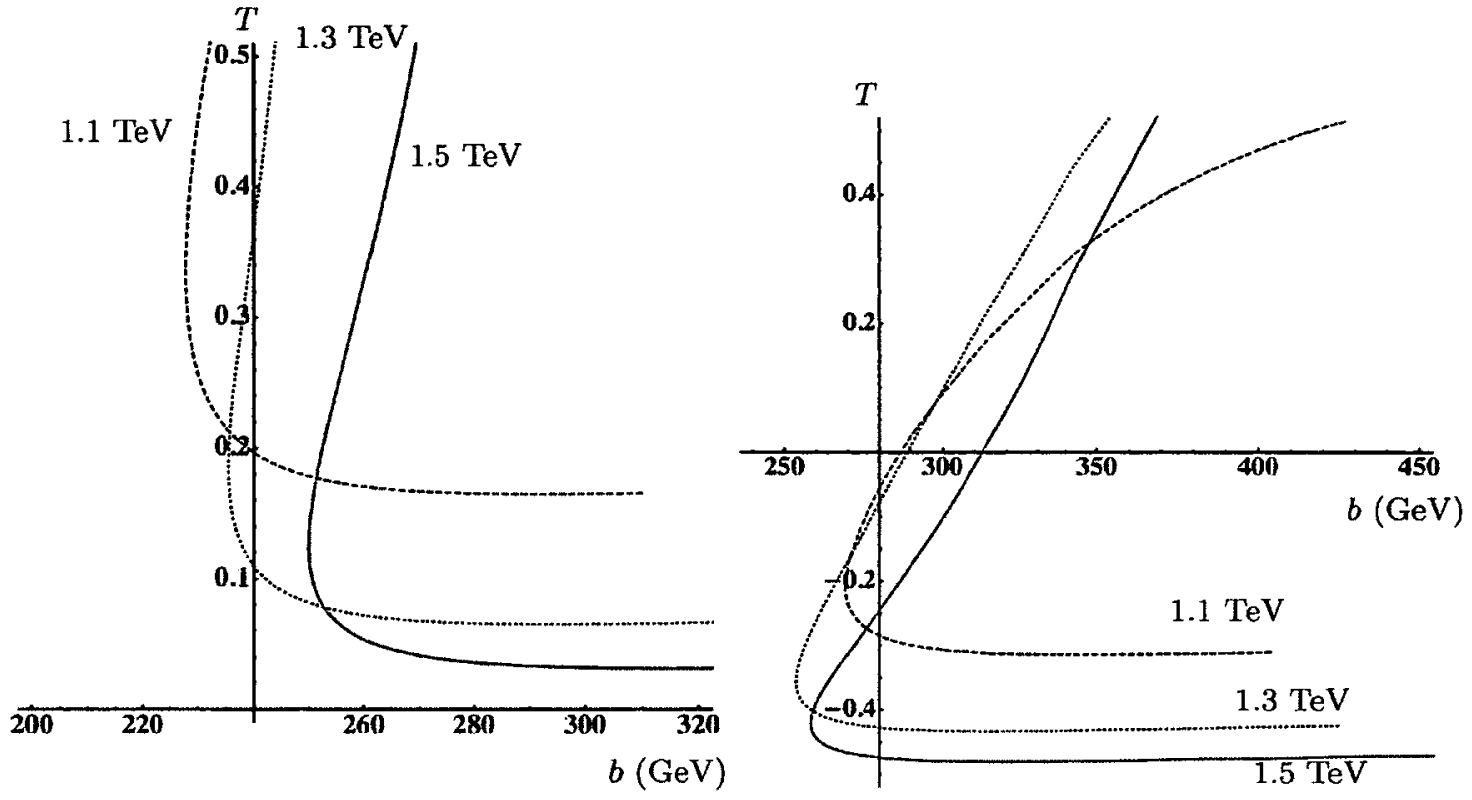

Figure 2.3: $T$ parameter contribution from the top sector as a function of $b$ for various $m_{U}$ and with $f=700 \mathrm{GeV}$. The plot on the left is for the $S U(6)_{L} \times S U(5)_{R}$ top sector with $m_{D}=1 \mathrm{TeV}$ while the plot on the right is for the $S U(6)$ top sector.

This, at one loop through a box diagram, gives a finite contribution to the four-fermion operator

$$
q_{\mathrm{SM}}^{\dagger} \sigma^{\mu} q_{\mathrm{SM}} q_{\mathrm{SM}}^{\dagger} \sigma_{\mu} q_{\mathrm{SM}}
$$

as found in [43]. It leads to the constraint: $k f_{k} \lesssim 1 \mathrm{TeV}$ if we assume an universal $k[43]$.

\subsection{Dark Matter}

In this model the T-parity is exact, and the lightest T-odd particle (LPOP) will be stable and will contribute to dark matter abundance. The model contains a few Todd particles with masses in the $\sim 100 \mathrm{GeV}$ range that could be the LPOP, namely the odd triplet and the odd Higgs doublet. We will consider the case where $\tilde{S}$, the CP-even component of the inert Higgs doublet is the dark matter. In our model 
$\tilde{S}$ is always lighter than $\tilde{A}$, and we can choose it to be lighter than $\tilde{h}^{+}$. The relic abundance is completely determined by the parameters of the Higgs potential and was computed for various choices of parameters in [39,44-47]. Using micrOMEGAs [48] we calculated the relic abundance for parameters that are appropriate for our model, namely a nearly degenerate CP-even and charged T-odd states and masses of the Higgses doublet related as in (2.73). We fix $m_{h}$ at $120 \mathrm{GeV}$ and vary $m_{\tilde{S}}$ and $\delta$ (see (2.74)). The coupling $\lambda_{3}$, which in our case control the coupling of two $\tilde{S}$ 's to the Higgs and play an important role in the dark matter abundance calculation is taken to be $-2 \lambda_{1}=-m_{h}^{2} / v^{2}$. This is appropriate if $c_{3}$ is the larger than the other quartic couplings in the $h_{A}, h_{B}$ basis.

If $\tilde{S}$ is heavier than roughly twice the $Z$ mass, allowing its annihilation into a pair of gauge bosons it will lead to a dark matter abundance that is below the observed abundance for $m_{\tilde{S}}$ not too far than this threshold. For light $m_{\tilde{S}}$, the only possible annihilation channel is to $b \bar{b}$ in which case the cross section is too small and the relic abundance is too large. In between these two regions, there are typically regions where the relic abundance is correct. Also, if the parameters are such that $m_{\tilde{S}}$ can be near $m_{h} / 2$, but below $m_{W} / 2$, there in an additional enhancement of the annihilation cross section due to the possibility of exchanging an on-shell Higgs. As found also in [39] this lead to a possible dark matter candidate of mass $\sim 70 \mathrm{GeV}$ with a Higgs mass at $120 \mathrm{GeV}$. As a consequence of (2.73), this scenario would imply a light $m_{\bar{A}}$, which seem hard to accommodate with the calculable $b$ values presented in figure 2.1. Note however that other contribution to $b^{2}$ could be added. There is also a possibility of having a heavier dark matter candidate, with mass near $500 \mathrm{GeV}$ and a Higgs still around $120 \mathrm{GeV}$. This scenario would require a slight fine-tuning to get the light Higgs. In figure (2.4) we show contour plots that illustrate the allowed region for low and high $m_{\tilde{S}}$ masses.

Because $\tilde{S}$ and $\tilde{A}$ are split in mass, direct detection through a $Z$ exchange is 
small. Direct detection through a Higgs exchange could be large if $\left|\lambda_{3}\right|$ is large. In our case, because $c_{3}$ is the dominant coupling this would mean a high Higgs mass. Also if $\tilde{S}$ and $\tilde{h}^{+}$are degenerate enough, direct detection could proceed through inelastic scattering [49]. This would require a mass splitting of order $\sim 100 \mathrm{keV}$, which correspond to $\delta \lesssim 0.001$.
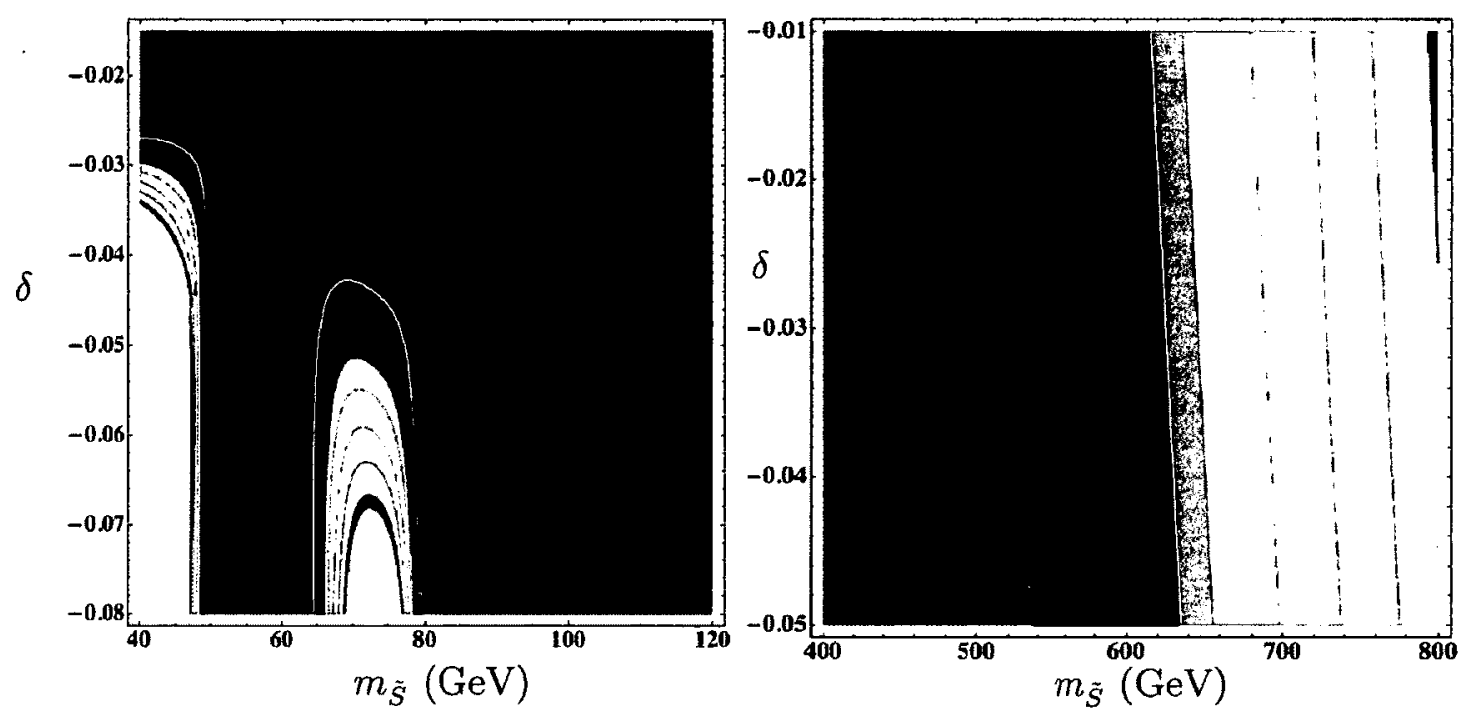

Figure 2.4: Contour plots of $\Omega h^{2}$ as a function of $m_{\tilde{S}}$ and $\delta$. The region in black correspond to the dark matter abundance measured by WMAP. The plot on the left shows a low $m_{\tilde{S}}$ region, while the plot on the right shows a high $m_{\tilde{S}}$ region, both have the Standard Model Higgs mass set at $120 \mathrm{GeV}$.

\subsection{Phenomenology}

The phenomenology of the $\sim \mathrm{TeV}$ scale particles of this model is similar to other Little Higgs models with T-parity $[43,50,51]$. There are odd and even top 'partners', the later being similar to that of Littlest Higgs or other Little Higgs model without T-parity and the former having signature potentially similar to the stop [52]. There are new odd gauge bosons, which would be produce in pair and also decay through a cascade leading to missing energy signatures. However, the cascade in this model will 
end-up in the Higgs sector with particles of masses of order $\sim 100 \mathrm{GeV}$, as opposed to T-odd gauge bosons of $\sim \mathrm{TeV}$ scale mass as is the case in the original Littlest Higgs model with T-parity.

The phenomenology of the odd doublet has been somewhat studied in the context of inert Higgs double models [53-55] and can have signatures similar to neutralino and charginos. In particular, it is possible to have tri-lepton signatures and Tevatron bounds on chargino translate into a bound of $\sim 70-90 \mathrm{GeV}$ for $m_{\tilde{h}^{+}}$in inert Higgs doublet models [39]. However, note that since our charged and neutral CP-even Higgs are almost degenerate, the kinematic of the decay might be different than the one studied in [39] and the bound might not apply in this case.

Finally, the new odd triplet could provide another handle to distinguish this kind of model from other T-parity models and would be interesting to study in more details.

\subsection{Conclusions}

Little Higgs models without T-parity suffer from important constraints on their parameter space due to deviations in electroweak precision tests. These constraints are eased significantly by the introduction of T-parity. The problem is that T-parity tends to introduce new structure in the theory. On the one hand, the fermion sector of T-parity invariant models needs a larger global symmetry structure, and contain possible new flavour spurions that need to be close to the identity [56]. The second issue concerns the implementation of T-parity in the UV theory, which was the motivation for the work we presented in this chapter.

The action of T-parity on the non-linear sigma model field of the Littlest Higgs model was originally proposed to be of the form:

$$
\Sigma \rightarrow \Omega \Sigma^{\dagger} \Omega \quad \text { with } \Omega \in S O(5)
$$


If $\Sigma$ is a composite of fermions, the corresponding symmetry in the UV theory is not straightforward to implement. The situation is in many ways similar to what happens in QCD. Looking only at the chiral Lagrangian, it seems easy to impose a $Z_{2}$ under which the pions and kaons are odd:

$$
\Sigma_{\mathrm{QCD}} \rightarrow \Sigma_{\mathrm{QCD}}^{\dagger}
$$

However, it is well known that this is not a symmetry of the QCD chiral Lagrangian. The kinetic term of the non-linear sigma model field is invariant, but there are terms, such as the $\pi^{0} F \tilde{F}$ term or the WZW term that break it and allow coupling of one $\pi$ to two photons, or two $\pi$ 's to three $K$ 's. In fact, the high energy Lagrangian containing the quarks and gluon has no symmetry that would lead to (2.83) at low energy. Similarly, a symmetry of the form shown in (2.82) can not be straightforwardly implemented in a strongly coupled UV completion such as the one of [25]. In this chapter, we examined the possibility of implementing T-parity in a different way, in the $S U(6) / S p(6)$ Little Higgs model. In this model the T-parity is an element of $S p(6)$ and could easily be implemented as an exchange symmetry in a strongly coupled UV completion. We did not build an explicit UV completion as it would present similar challenges as in other Little Higgs models, especially in the fermion sector. We instead focused on the description of the theory below $\sim 10 \mathrm{TeV}$.

One of the distinguishing feature of the model we presented is the presence at low energy of an inert two Higgs doublet model. The neutral state CP even state of the T-odd doublet is a potentially viable dark matter candidate, and would lead to interesting collider phenomenology, similar to gaugino production. The Higgs sector also respects an approximate custodial symmetry that makes the CP even neutral Higgs and the charged Higgs quite degenerate. It prevents this sectors from giving large contributions to the $T$ parameter and could lead to interesting effects at colliders. To give Yukawa couplings to fermions in a T-parity invariant way, we introduced a 
new $S U(2)$ gauge symmetry as well as two 'link' fields that break the resulting $S U(2)^{3}$ gauge symmetry down to the diagonal subgroup. The extra gauge field can be made heavy, but the link fields contain an extra T-odd scalar triplet which get a mass in the $\sim 100 \mathrm{GeV}$ range. The presence of such a triplet coming from link fields seem to be a generic feature of simple group Little Higgs model with T-parity [34]. It could also be a dark matter candidate, and its phenomenology would be interesting to study further. This model could be consistent with electroweak precision data, and yield the correct dark matter abundance. 


\section{Chapter 3}

\section{Supersymmety}

We have explained in the first chapter that supersymmetry (SUSY) provides a possible solution to the hierarchy problem. In this chapter we will first give an introduction to the supersymmetric formalism ${ }^{1}$, then we will discuss the MSSM, the Minimal Supersymmetric extension of the Standard Model, and finally we will introduce a less minimal SUSY extension of the SM (SSM) where the gauginos are Dirac fermions instead of Majorana fermions like in the MSSM.

\subsection{Supersymmetric algebra, Superspace and Su- perfields}

Supersymmetry is a symmetry which connect bosons and fermions (see [57], [59] and [58] for extensive reviews). Since its generators $Q$ turn a bosonic state into a fermionic one, and vice-versa, they must carry half integer spin. This means that SUSY generators are fermionic, while the generators of the Lorentz group or of a gauge group are bosonic. In a 4-dimensional spacetime the minimal spinor is a Weyl

\footnotetext{
${ }^{1}$ We will focus on a $N=1$ supersymmetry as it is the most relevant on a phenomenological point of view.
} 
spinor and therefore the minimal supersymmetry has 4 supercharges, that is a 2component (Weyl) spinor $Q$ and its conjugate $\bar{Q}$, and their algebra is:

$$
\begin{gathered}
\left\{Q_{\alpha}, Q_{\beta}\right\}=\left\{\bar{Q}_{\dot{\alpha}}, \bar{Q}_{\dot{\beta}}\right\}=0, \\
\left\{Q_{\alpha}, Q_{\dot{\beta}}\right\}=2 \sigma_{\alpha \dot{\beta}}^{\mu} P_{\mu}, \\
{\left[Q_{\alpha}, P_{\mu}\right]=0 .}
\end{gathered}
$$

where $\{$,$\} denotes the anti-commutator, the indices \alpha, \beta$ of $Q$ and $\dot{\alpha}, \dot{\beta}$ of $\bar{Q}$ goes from 1 to 2 , and $\sigma^{\mu}=\left(\mathbf{1}, \sigma_{i}\right)$ where $\sigma_{i}$ are the Pauli matrices. $P_{\mu}$ is the generator of translations. Supersymmetry is an extension of the spacetime symmetry which can be realized on ordinary fields (functions of spacetime) by transformations that mix bosons and fermions. However, as always happen when a new framework is proposed, it is convenient to introduce a new formalism: the superspace and superfield approach. Superspace is an extension of the ordinary spacetime to include extra anticommuting coordinates in the form of two-component Weyl spinors $\theta$. The superfields $\psi(x, \theta, \bar{\theta})$ are functions defined over this space. They can be expanded in a Taylor series with respect to the anticommuting coordinates $\theta$; since the square of an anticommuting quantity vanishes, this series has only a finite number of terms. The coefficients obtained in this way are the ordinary component fields.

We start introducing the chiral superfields $\Phi(x, \theta)$ which contain the following degrees of freedom:

- $\phi(x)$ a complex scalar,

- $\psi(x)$ a Weyl spinor,

- $F(x)$ a complex auxiliary field.

The superfield $\Phi$ contains 4 bosonic degrees of freedom, but just two of them, $\phi$, are physical, and they match the 2 fermionic degrees of freedom $\psi$. As we said above, we 
can expand a superfield in a Taylor series with respect the variable $\theta$ obtaining:

$$
\Phi(y, \theta)=\phi(y)+\sqrt{2} \theta^{\alpha} \psi_{\alpha}(y)+\theta^{\alpha} \theta^{\beta} \epsilon_{\alpha \beta} F(y)
$$

where $y=x+i \theta \sigma \bar{\theta}$, and which is an exact expansion since $\theta$ has just two components and the square of any component vanishes which implies that there are not terms with three or more factors of $\theta$. It is important to notice that $\theta$ has mass dimension $-1 / 2$ so then the auxiliary field $F$ has dimension 2 , and the superfield itself dimension 1. As expected, under a supersymmetry transformations the scalar component of $\Phi$ transforms into the fermionic superpartner:

$$
\delta \phi=\sqrt{2} \psi \alpha
$$

and vice-versa:

$$
\delta \psi=\sqrt{2} \alpha F+i \sqrt{2} \sigma_{\mu} \bar{\alpha} \phi^{\mu}
$$

while the auxiliary field transforms into a total derivative:

$$
\delta F=-i \sqrt{2} \partial_{\mu} \psi \sigma_{\mu} \alpha
$$

Therefore the quantity $\int d^{4} x F(x)$ is invariant under SUSY. Having generalised the field concept we now need to explain how to build a SUSY invariant Lagrangian out of chiral superfields. Invariance under supersymmetry requires the Lagrangian $\mathcal{L}$ to transform into a total derivative. We define a new function, the superpotential $W$, which is an analytic function of chiral superfields, $W=W\left(\Phi_{i}\right)$, which can be shown to be a chiral superfield. Therefore, since the auxiliary component of a superfield transforms like a total derivative, $\mathcal{L}_{\mathcal{W}}=\int d^{4} x\left(\int d^{2} \theta W\left(\Phi_{i}\right)\right)$ is the contribution to the Lagrangian which contains interactions such as for example the Yukawa interactions, and the mass terms for the chiral superfields of the theory. However $\left.W\right|_{\theta^{2}}$ does not contain space-time derivative necessary for the Lagrangian kinetic terms. We define 
then another function, the Kahler potential $K$ :

$$
K=\sum_{i} \Phi_{i}^{\dagger} \Phi_{i}
$$

and then the corresponding Lagrangian is $\mathcal{L}_{\mathcal{K}}=\int d^{4} \theta K$ is also invariant under SUSY. In order to write down a full SUSY Lagrangian the last ingredient to introduce is the vector superfields $V$, namely the superfield which describes the spin 1 gauge bosons. A vector superfield $V=V^{\dagger}$ contains:

- $A_{\mu}^{a}(x)$ real vector bosons,

- $\tilde{A}^{a}(x)$ Weyl spinors called gauginos,

- $D(x)^{a}$ which are real auxiliary fields.

Therefore, considering for simplicity an abelian $U(1)$ gauge theory, the supersymmetric Lagrangian for a vector supefield is:

$$
\mathcal{L}=\left(\int d^{2} \theta \frac{1}{4 g^{2}} \mathcal{W}^{\alpha} \mathcal{W}_{\alpha}+\int d^{2} \bar{\theta} \frac{1}{4 g^{2}} \mathcal{W}^{\dot{\alpha}} \mathcal{W}_{\dot{\alpha}}\right),
$$

where $\mathcal{W}_{\alpha}=-\frac{1}{4} \bar{D}^{2} D_{\alpha} V$ is a chiral superfield.

Finally under the gauge transformation a chiral superfield $\Phi$ of charge $q$ transforms in the following way:

$$
\Phi \rightarrow e^{-i q \Lambda} \Phi
$$

where $\Lambda$ is a chiral superfield. The canonical Kahler potential is defined as:

$$
K=\Phi^{\dagger} e^{q V} \Phi,
$$

and it contains the regular gauge interactions together with new 'SUSY' interactions of matter fields with gauginos:

$$
-\sqrt{2}\left(\phi^{*} \psi \tilde{A}+\tilde{A}^{\dagger} \psi^{\dagger} \phi\right)
$$

tipically called the gauge Yukawa. 


\section{$3.2 \quad R$-symmetry}

A $U(1)_{R}$ symmetry is a $U(1)$ symmetry which rotates superspace coordinates $\theta$ by a phase:

$$
\theta \rightarrow e^{i \eta} \theta
$$

and under which a chiral superfield transform as:

$$
\Phi \rightarrow e^{i 2 \eta R} \Phi
$$

where $R$ is the $U(1)_{R}$ charge of $\Phi$. The $R$-symmetry is therefore a global symmetry that, unlike all the others, acts on the superspace coordinates $\theta$ and $\bar{\theta}$. As a result different components of the superfields transform differently under the $R$-symmetry. Consider, for example, a chiral superfield with $R$-charge $R$. Its lowest component has the same $R$-charge as the superfield itself, its $\theta$ component has $R$-charge $R-1$ and its $\theta^{2}$ component has $\mathrm{R}$-charge $R-2$. The $R$-charges of matter fields depend on the model under consideration, while the $R$ charges of the fields in a vector multiplet

are uniquely fixed. Indeed $W^{\mu} W_{\mu}$ must have $R$-charge 2 in order for eq.(3.9) to be invariant under the $R$-symmetry. Since the gaugino is the lowest component of $W$ its R-charge is 1 while the D-term and the gauge field $A^{\mu}$ are neutral (as should have been expected for real fields). To build a $U(1)_{R}$ invariant Lagrangian, the Kahler potential should have $R$-charge 0 , but since $\theta$ transforms as eq.( 3.13 ) the superpotential $W$ must have $R$-charge 2 .

\subsection{MSSM}

The Minimal Supersymmetric Standard Model (MSSM) is the extension of the Standard Model with the most minimal particle content allowed by supersymmetry. It contains the superfields corresponding to the SM degrees of freedom, i.e. the SM particles and their superpartners plus an enlarged Higgs sector. The scalar superpartners 
of the quarks are called squarks and the superpartners of the leptons sleptons. A supersymmetric Higgs sector must contain two Higgs doublets, while in the SM it is sufficient to only have one. In the MSSM we need two Higgs doublets $H_{u}$ with hypercharge $1 / 2$ and $H_{d}$ with hypercharge $-1 / 2$ in order to give mass to the up type and the down type SM fermions and in order to cancel the gauge anomalies associated with triangle diagrams. The reason is that a supersymmetric Higgs superfield also contains fermionic degrees of freedom, the higgsino $\tilde{h}$, which introduces new anomalies. These can be cancelled by adding a second Higgs doublet with opposite hypercharge. It turns out that this extra Higgs doublet with opposite hypercharge, and therefore with the same gauge quantum numbers as the lepton doublet, is also needed to give mass to the down type fermions. Indeed, as we have explained in the previous section, the superpotential is an holomorphic function of chiral superfields. This implies that a term such $H_{u}^{\dagger} L e^{c}$ is not allowed and therefore in order to give mass to the down type fermions a higgs doublet with hypercharge $-1 / 2$ is required. The superpotential of the MSSM is then given by the extremely simple expression:

$$
W_{M S S M}=Y_{u} U^{c} Q H_{u}-Y_{d} D^{c} Q H_{d}-Y_{e} E^{c} L H_{d}+\mu H_{u} H_{d}
$$

The superpotential in eq.(3.15) contains all the terms necessary to build a viable SUSY extensions of the SM, but it is not the most general superpotential one can write down with the MSSM particle content. Other terms are allowed by the gauge invariance and by the analyticity requirements for $W$, namely:

$$
\begin{gathered}
W_{\Delta L=1}=\frac{1}{2} \lambda^{i j k} L_{i} L_{j} E_{k}^{c}+\lambda^{\prime i j k} L_{i} Q_{j} D_{k}^{c}+\mu_{i} H_{u} L_{i} \\
W_{\Delta B=1}=\frac{1}{2} \lambda^{\prime \prime i j k} U_{i}^{c} U_{j}^{c} D_{k}^{c}
\end{gathered}
$$

These terms violate either the lepton number $\mathrm{L}, W_{\Delta L=1}$, or the baryon number, $W_{\triangle B=1}$. Therefore in the MSSM, lepton and baryon numbers are not accidental 
symmetries as in the SM. The presence of these terms is potentially dangerous since lepton and baryon number violating processes have not been experimentally observed. In particular unsuppressed $\lambda^{\prime}$ and $\lambda^{\prime \prime}$ together could lead to a short proton lifetime. Therefore, it is important to take care of the existence of these extra terms in the superpotential. The typical way out to this problem is simply to get rid of these additional contributions imposing a discrete symmetry, called $R_{p}$ parity which is defined as:

$$
R_{p}=(-1)^{3(B-L)+2 s} .
$$

The $R_{p}$ parity is a $Z_{2}$ subgroup of a $U(1)_{R}$ symmetry with $R$-charge assignement for the MSSM superfields such that all the SM matter fields and the Higgses fields have $R$ charge zero. This implies that under the discrete symmetry $R_{p}$ all the superpartners of the SM fermions and the higgsinos are odd, while the SM particles are even. This deeply affects the phenomenology of the MSSM with $R_{p}$ since for example all the superpartners can only be pair produced. Furthermore, the lightest among the $R_{p}$ odd particles is stable and therefore, is a dark matter candidate of the model.

\subsection{Susy breaking}

Phenomenological models like the MSSM cannot be completely supersymmetric since we did not discover the SM scalar superpartners which if SUSY was exact would have the same mass as their fermionic partner. Therefore, SUSY must be broken, but its breaking has to be soft. This means that SUSY breaking should not reintroduce quadratic divergences if one consider the solution of the hierarchy problem as the main motivation for SUSY. This could be achieved if SUSY is spontaneously broken. One could attempt to construct extensions of the MSSM with spontaneously broken supersymmetry. However, if the MSSM superfields have renormalizable coupling to 
SUSY breaking the tree level spectrum of such models would satisfy the supertrace condition, $\operatorname{Str} M=0$. While the supertrace condition is modified by quantum effects, within MSSM alone such modifications are small since the Standard Model is a weakly interacting theory at the EWSB scale. Since the Standard Model fermions are generally light, the supertrace condition requires the existence of new light bosons which have not been observed experimentally. We conclude that SUSY must be broken in a different, hidden, sector of the theory. In order to produce a viable spectrum this hidden sector should have no (or only very small) direct couplings to the the MSSM. However, the two sectors do share some interactions that are responsible for mediating supersymmetry breaking from the hidden sector to the visible sector, resulting in the MSSM soft terms. On the phenomenological point of view it is important then to specify how SUSY is mediated to the MSSM rather than understanding how it is broken.

The main idea for the SUSY breaking mediation mechanism are:

- Planck-scale-mediated supersymmetry breaking (PMSB): in this framework the hidden and the visible sector communicated just through gravity. Therefore, SUSY breaking is mediated to the visible sector through higher dimensional operators suppressed by powers of the Planck scale.

- Gauge mediated supersymmetry breaking (GMSB): in this framework one assumes that SUSY breaking is communicated to the standard model via heavy chiral supermultiplets that are charged under the standard model gauge symmetries. If the masses of these messenger fields are not exactly supersymmetric, integrating them out will give rise to SUSY breaking in the visible sector.

- Anomaly mediated supersymmetry breaking (AMSB): this is a special case of gravity mediation where there is no direct tree level coupling that transmits the SUSY breaking in the hidden sector to the observable one. 
The spontaneous breaking of global supersymmetry implies the existence of a massless Weyl fermion, the goldstino. The goldstino is the fermionic component of the supermultiplet whose auxiliary field obtains a VEV, it is then the equivalent of the Goldstone boson for a ordinary global symmetry. In locally supersymmetric theories once supersymmetry is spontaneously broken, the gravitino acquires a mass by absorbing (eating) the goldstino, which becomes its longitudinal (helicity $\pm 1 / 2$ ) components. This is analogous to the ordinary Higgs mechanism for gauge theories, by which the $W$ and $Z$ gauge bosons in the Standard Model gain mass by absorbing the Nambu-Goldstone bosons associated with the spontaneously broken electroweak gauge invariance. The massive spin $3 / 2$ gravitino now has four helicity states, of which two were originally assigned to the would-be goldstino.

\subsubsection{MSSM soft SUSY breaking terms}

The soft breaking terms we can write down out of the MSSM particle content are:

- gaugino Majorana masses: $M_{a} \tilde{A}_{a} \tilde{A}_{a}$,

- scalar masses: $m^{2} \phi^{\dagger} \phi$,

- triple scalar interactions $a_{i j k} \phi_{i} \phi_{j} \phi_{k}$,

- Higgs bilinear: $b_{\mu} h_{u} h_{d}$.

Therefore, the SUSY breaking Lagrangian for the MSSM is:

$$
\begin{aligned}
\mathcal{L}_{\text {soft }}^{M S S M}= & -\frac{1}{2}\left(M_{\tilde{g}} \tilde{g} \tilde{g}+M_{\tilde{W}} \tilde{W} \tilde{W}+M_{\tilde{B}} \tilde{B} \tilde{B}+h . c .\right) \\
& -\tilde{Q}^{\dagger} m_{Q}^{2} \tilde{Q}-\tilde{L}^{\dagger} m_{L}^{2} \tilde{L}-\tilde{u}^{\dagger} m_{u}^{2} \tilde{u}-\tilde{d}^{\dagger} m_{d}^{2} \tilde{d}-\tilde{e}^{\dagger} m_{e}^{2} \tilde{e} \\
& -\left(\tilde{u} A_{u} \tilde{Q} H_{u}-\tilde{d} A_{d} \tilde{Q} H_{d}-\tilde{e} A_{e} \tilde{L} H_{d}+h . c .\right) \\
& -m_{H_{u}}^{2} H_{u}^{\dagger} H_{u}-m_{H_{d}}^{2} H_{d}^{\dagger} H_{d}-\left(b H_{u} H_{d}+\text { h.c. }\right)
\end{aligned}
$$


where $A_{f}$ and $m_{f}^{2}$ are matrices in flavour space. Soft SUSY breaking introduces many free parameters in the MSSM. However, there are many requirements that these parameters should respect, especially from flavor physics, which allows to make simplifying assumptions and study the MSSM phenomenology.

\subsection{Natural MSSM and the LHC}

The MSSM is the most popular BSM model. Many of the LHC searches have been designed to look for it, and therefore, restriction on its parameter space after the first two years of LHC running are well studied. The limits on the masses of the first and second generation squarks and of the gluino have now almost reached the $\mathrm{TeV}$ threshold. This has several consequences especially if we believe that the main motivation for supersymmetry at the $\mathrm{TeV}$ scale should be the solution of the hierarchy problem. An heavy gluino is not natural since gluinos give a large one loop correction to the stop ( the top partner ) masses and therefore they indirectly give a large contribution to the Higgs mass ( see [60]). Indeed the one loop radiative corrections to $m_{H_{u}}^{2}$ proportional to the Yukawa coupling are given by:

$$
\delta m_{H_{u}}^{2}=-\frac{3}{8 \pi^{2}} y_{t}^{2}\left(m_{Q_{3}}^{2}+m_{u_{3}}^{2}+\left|A_{t}\right|^{2}\right) \log \left(\frac{\Lambda}{\mathrm{TeV}}\right)
$$

where $\Lambda$ is the SUSY breaking mediation scale, and $m_{Q_{3}}, m_{u_{3}},\left|A_{t}\right|$ are the parameters which determine the stop sector spectrum. Therefore if one inserts the gluino one loop contribution to the stop masses into eq.(3.20) obtains at the leading log (LL) approximation:

$$
\delta m_{H_{u}}^{2}=-\frac{2 y_{t}^{2}}{\pi^{2}}\left(\frac{\alpha_{s}}{\pi}\right)\left|M_{\tilde{g}}\right|^{2} \log ^{2}\left(\frac{\Lambda}{\mathrm{TeV}}\right)
$$


where $M_{\tilde{g}}$ is the gluino mass, eq.(3.20). This translates to a naturalness bound on the gluino mass, that is:

$$
M_{\tilde{g}} \leq 900 \mathrm{GeV} \sin \beta\left(\frac{\log \frac{\Lambda}{\mathrm{TeV}}}{3}\right)^{-1}\left(\frac{m_{h}}{125 \mathrm{GeV}}\right)\left(\frac{\Delta^{-1}}{20 \%}\right)^{-1}
$$

where $\Delta$ measures the fine tuning. Therefore, to a $900 \mathrm{GeV}$ gluino corresponds a $20 \%$ fine-tuning, and the LHC is then exploring the boundaries of a natural MSSM spectrum.

Therefore, even though there is still room for a natural MSSM ( see for example [60])), this motivates the interest to explore scenarios beyond the MSSM. In the following sections we will focus on a scenario where the gauginos are Dirac fermions instead of Majoranas as in the MSSM ( [62], [63], [64]) . One of the main advantages of this framework is that Dirac gluinos can be naturally heavier than Majorana gluinos. Furthermore, a large Dirac mass suppresses the production cross section of colored superpartners. This allows to have lighter first and second generation squarks, around $600-800 \mathrm{GeV}$, than in an equivalent MSSM spectrum as we will see in Chapter 6 ( see also [66] and [65] ).

\subsection{Dirac gauginos and Supersoft Supersymmetry breaking}

Dirac masses for gauginos require the extension of the MSSM particle content by adding adjoint chiral superfields for each SM gauge group, that is a singlet $\Phi_{\bar{B}}$, a triplet $\Phi_{\tilde{W}}$ and an octet $\Phi_{\tilde{g}}$. This corresponds to a $N=2$ supersymmetry particle content in the gauge sector. With this particle content we can write down Dirac masses for the gauginos, that is:

$$
\mathcal{L}_{f}=M_{\tilde{B}} \tilde{B} \psi_{\tilde{B}}+M_{\tilde{W}} \tilde{W} \psi_{\tilde{W}}+M_{\tilde{g}} \tilde{g} \psi_{\tilde{g}} .
$$


The interesting scenario for LHC phenomenology corresponds to the limit where the Majorana masses for the adjoints and the gauginos are suppressed compared to the Dirac masses. If, for example, one assumes that the supersymmetry breaking preserve an $R$-symmetry this condition is fulfilled. As we explained in the previous section, gauginos have $R$ charge 1, and therefore a Majorana mass term breaks such symmetry. We will enter in the details of the relation between Dirac gauginos masses and $R$ symmetry in a next section. Now we want to explain how Dirac masses for the gauginos are generated.

If $X$ is a chiral superfield with an $F$ term spurion, a gaugino Dirac mass can be generated through the following operator:

$$
\int d^{2} \theta \frac{1}{M^{3}} \bar{D}^{2} D_{\alpha}\left(X^{\dagger} X\right) W_{i}^{\alpha} \Phi_{i}
$$

where $i$ is the gauge group index and $M$ is the messenger scale. The Dirac mass generated in this way is subleading in an expansion in the SUSY breaking parameter $\frac{F}{M^{2}}$ since it is of order $\frac{F^{2}}{M^{3}}$. A possible way out is to have a low messenger scale $F \sim M^{2}$ (see also [67]).

Dirac masses for gauginos can also arise via an interaction with a hidden sector $U(1)$ gauge field which obtains a $\mathrm{D}$-term $\mathrm{VeV}$, and this is the scenario we will consider from now on ( see [64]). The Dirac masses are generated through the following operator:

$$
\int d^{2} \theta \frac{1}{M} W_{\alpha}^{\prime} W_{i}^{\alpha} \Phi_{i}
$$

The following operators can also be written down:

$$
\begin{array}{r}
\int d^{2} \theta \frac{W_{\alpha}^{\prime} W^{\prime} \alpha}{M^{2}} \Phi_{i}^{\dagger} \Phi_{i} \\
\int d^{2} \theta \frac{W_{\alpha}^{\prime} W^{\prime \alpha}}{M^{2}} \Phi_{i}^{2}
\end{array}
$$

which generate holomorphic and non holomorphic mass terms for the scalar components of the adjonts:

$$
\mathcal{L}_{s}=m_{\phi_{\bar{B}}}^{2} \tilde{\phi}_{\tilde{B}}^{\dagger} \tilde{\phi}_{\tilde{B}}+m_{\phi_{\tilde{W}}}^{2} \tilde{\phi}_{\tilde{W}}^{\dagger} \tilde{\phi}_{\tilde{W}}+m_{\phi_{\tilde{g}}}^{2} \tilde{\phi}_{\tilde{g}}^{\dagger} \tilde{\phi}_{\tilde{g}}+B_{\phi_{\tilde{B}}} \tilde{\phi}_{\tilde{B}}^{2}+B_{\phi_{\tilde{W}}} \tilde{\phi}_{\tilde{W}}^{2}+B_{\phi_{\bar{g}}} \tilde{\phi}_{\tilde{g}}^{2}
$$


The operator in eq.(3.25) is supersoft which means that it does not induce log divergent contributions to the other soft parameters. Indeed it contains the following terms:

$$
\mathcal{L} \supset-M_{D} \lambda_{i} \psi_{i}-\sqrt{2} M_{D}\left(\tilde{\phi}_{i}+\tilde{\phi}_{i}^{*}\right) D_{i}-D_{i}\left(\sum_{j} g_{j} \tilde{f}_{j}^{*} t_{i} \tilde{f}_{j}\right)-\frac{1}{2} D_{i}^{2}
$$

where $\tilde{\phi}$ is the complex scalar component of the adjoint superfield, and $\tilde{f}$ represents all the scalar fields charged under the gauge group $G_{i}$. Once equations of motion are used to replace $D_{i}$, eq.(3.29) contains an interaction term between the sfermions and the scalar adjoint $\tilde{\phi}$. This gives rise to a new contribution to the one loop radiative corrections to the mass of the scalars $\tilde{f}$ which cancel the log divergence of the gaugino radiative correction to the scalar mass leaving the following finite mass contribution for the sfermion [64]:

$$
m_{s}^{2}=\sum_{b=1}^{3} \frac{C_{s}^{b} \alpha_{b} M_{b}^{2}}{\pi} \log \frac{m_{\phi_{b}^{R}}^{2}}{M_{b}^{2}}
$$

where $C_{s}^{b}$ is the quadratic Casimir of the scalar $s$ under the group $b$, that is equal to $Y^{2}(s)$ for $U(1)_{Y}$, and $\frac{N^{2}-1}{2 N}$ for $S U(N)$. These contributions to scalars masses are positive and flavor blind since they arise from gauge interactions. One can therefore consider a spectrum where the sfermion masses are generated through eq.(3.30)in a sort of gaugino mediation. These masses are a loop factor suppressed with respect to the gaugino masses, and this supersoft spectrum is characterised by gauginos heavier than scalars. Furthermore, the term in eq.(3.30) is completely UV insensitive, and this can reduce the fine tuning. Indeed the stop contribution to the Higgs mass is in this scenario:

$$
\delta m_{H_{u}}^{2}=\frac{3 y_{t}^{2}}{4 \pi^{2}} M_{\tilde{t}}^{2} \log \frac{M_{\tilde{g}}^{2}}{m_{\tilde{t}}^{2}}
$$

where $M_{\tilde{g}}$ is the gluino mass since in this scenario this is the scale where the stop acquire a mass. This is an important difference with the MSSM where the cutoff is 
necessarily the UV cutoff, that is an higher scale. Therefore, in a supersoft spectum the stop can potentially be heavier than in the MSSM without increasing the fine tuning of the model. However, eq.(3.30) gives problematically-light sleptons, which typically lead to consider combined F- and D-term SUSY breaking, of the same or$\operatorname{der}[68]$. If one then consider other contributions to the stop mass, the stop corrections to the Higgs mass are the same as in the MSSM.

In general, there are a few problems associated with Dirac gauginos which are important to mention and which represent a challenge for model building. First the adjoints scalars turn out to be tachyonic in the context of minimal gauge mediation. This is because typically $B_{\Phi}>m_{\Phi}^{2}$. This problem was solved in [68] by a judicious

choice of adjoints couplings to the messengers. Furthermore, the bino adjoints is a pure singlet and therefore it can in principle acquire a dangerous tadpole term in the potential. Finally the chiral adjoints drastically alter the usual picture of gauge coupling unification. Therefore, the minimal spectrum for Dirac gauginos does not give rise to unification. However, many scenarios for adding additional matter have been proposed which could help unify the couplings [68].

\subsubsection{Supersoft terms and natural SUSY}

As we have already explained in the previous chapter, the LHC is putting strong bound on the MSSM SUSY particle and this motivates the effort to understand which are the upper bounds on the sparticle masses compatible with naturalness . The naturalness bound for the gluino is at around $900 \mathrm{GeV}[60]$. Therefore, the LHC is now probing the limit of the natural MSSM spectrum. Dirac gauginos instead can be naturally heavier than Majorana gauginos since they are generated through supersoft operators, [66]. If we insert eq.(3.30) in the one loop stop contribution to 
the Higgs mass eq.(3.20), we obtain:

$$
\delta m_{H_{u}}^{2} \sim \frac{y_{t}^{2} \alpha_{s} M_{\tilde{g}}^{2}}{\pi^{3}} \log \frac{M_{\tilde{g}}^{2}}{m_{\tilde{t}}^{2}} \log r_{3}
$$

where $r_{3}=\frac{M_{\bar{g}}}{m_{\phi \frac{R}{g}}}$. We have assumed that the stop receive mass just through the finite gaugino contributions, eq.(3.30). By comparing eq.(3.32) with eq.(3.21) it is clear that the naturalness bound on Dirac gluinos is higher. Indeed a $5 \mathrm{TeV}$ Dirac gluino with $r_{3} \sim 1.5$ is as natural as a $900 \mathrm{GeV}$ Majorana gluino with $\Lambda \sim 20 \mathrm{TeV}$ as it has been recently discussed in [66].

\subsection{2 $U(1)_{R}$ symmetry: another motivation for Dirac gaugi- nos}

Dirac gaugino masses preserve an $R$-symmetry in contrast to Majorana gauginos masses. This allows then to build models which posses a $U(1)_{R}$ symmetry, and this is an interesting possibility to explore for several reasons. However, the $R$-symmetry cannot be an exact symmetry because it is broken (at least) by the gravitino mass term that is necessary to cancel the cosmological constant.

One of the traditional motivation for $R$ symmetric models is related to SUSY breaking. Indeed it has been proven that under certain general conditions ${ }^{2}$ the existence of an $R$-symmetry in the hidden sector is a necessary condition for sponteanous supersymmetry breaking [69]. Furthermore, the existence of an $R$-symmetry ameliorates the SUSY flavor problem and CP problem, [70]. This allows the possibility to have a significant flavor violation especially in the squark sector ${ }^{3}$, and could accomodate a realistic gravity mediated SUSY breaking which are otherwise plagued by flavor problems, [72]. This is also a very interesting possibility because the LHC is now pushing the first and the second generation squark masses above the $\mathrm{TeV}$ threshold. Since the

\footnotetext{
${ }^{2}$ such as the assumption of a generic superpotential

${ }^{3}$ In the slepton sector the flavor violation cannot be maximal since the $R$-symmetry is not enough to suppress lepton flavor violation processes [71]
} 
third generation squarks are required to be at the electroweak scale by naturalness the LHC bounds point towards a flavorful SUSY mediation mechanism. This could create tension with flavor physics unless the first generation squarks are extremely heavy, and this could reintroduce tension with naturalness. Also it would be difficult to motivate such a huge hierarchy in the squarks spectrum. For this reason it would be interesting to explore flavorful SUSY mediation models with $R$-symmetry. We plan to explore this in a future work.

Another motivation to have a quasi exact $R$-symmetry is that since it allows the possibility to have a larger CP violation this could be useful to build models of electroweak baryogenesis [73].

\section{7 $\quad$ Models with a quasi exact $R$-symmetry}

In order to build a realistic model with a quasi exact $U(1)_{R}$ symmetry the first step is to determine which are the $R$-charges of SM superfields. The SM particle should have $R$-charge zero unless the $R$-charge is identified with a global SM symmetry such lepton number or baryon number. If we require all the SM particles to be neutral under a $U(1)_{R}$ the usual $R_{p}$ will be a $Z_{2}$ subgroup of this $U(1)_{R}$. This corresponds to the typical definition of the $R$-symmetry, and a complete model with such $U(1)_{R}$ symmetry was built in [70] which we will summarise briefly in the next section. In [2] 4 instead the $R$ symmetry were indentified with the lepton number, and this is the scenario we will focus on in the following chapters. Another interesting possibility, which however has not been fully explored yet, is to identify the $R$-symmetry with the baryon number instead [61].

\footnotetext{
${ }^{4}$ see [74] for an early implementation of the idea of an $R$ symmetry lepton number.
} 


\subsubsection{The MRSSM}

In this section we will discuss the main features of the MRSSM (Minimal R-symmetric Supersymmetric Extension of the Standard Model) which has been proposed in [70]. As we have explained in the previous section, models with Dirac gauginos have an enlarged particle content with respect to the MSSM since they contain an adjoint chiral superfield $\Phi_{i}$ for each SM gauge group $G_{i}=S U(3)_{C}, S U(2)_{L}, U(1)_{Y}$. The $R$-symmetry also forbids the $\mu$ term: indeed the $R$-charge for the Higgs superfields should be 0 in order not to break the $R$-symmetry spontaneously, but this choice forbids the $\mu$ term $H_{u} H_{d}$ in the superpotential since the $R$-charge of the superpotential should be 2. In [70] this problem is solved by enlarging the Higgs sector, shown in table 3.1 where we present the MRSSM particle content. The Higgs sector contains indeed four doublets: $H_{u}, H_{d}, R_{u}$ and $R_{d}$ two of them, $H_{u}$ and $H_{d}$ have $R$-charge 0 , acquire a vev and give mass to the up type and down type fermions. The other two $R_{d}$ and $R_{u}$ have instead $R$-charge 2 so they are inert doublets, and they couple with the two Higgs superfields through the $\mu$ terms, that is $\mu_{u} R_{d} H_{u}+\mu_{d} R_{u} H_{d}$. the $R$ charge assignments is chosen such that all the Standard Model particles carry $R$-charge zero.

With this particle content the MMRSSM superpotential is then:

$$
W=\mathbf{y}_{u} U^{c} Q H_{u}-\mathbf{y}_{d} D^{c} Q H_{d}-\mathbf{y}_{l} E^{c} L H_{d}+\mu_{u} H_{u} R_{d}+\mu_{d} H_{d} R_{u}
$$

where $\mathbf{y}_{u}, \mathbf{y}_{d}$ and $\mathbf{y}_{l}$ are $3 \times 3$ matrices in family space. The parameter space of the MRSSM is then larger than the already large parameter space of the MSSM. We could also add to the superpotential the following additional interactions:

$$
\lambda_{S} H_{u} R_{d} \Phi_{\bar{B}}+\lambda_{T} H_{u} R_{d} \Phi_{\bar{W}}
$$

These could interesting to raise the Higgs mass up to $125 \mathrm{GeV}$, but the discussion of the Higgs sector is beyond the purpose of our discussion. 


\begin{tabular}{l|l|l} 
SuperField & $\left(S U(3)_{c}, S U(2)_{L}\right)_{U(1)_{Y}}$ & $U(1)_{R}$ \\
\hline$Q_{i}$ & $(3,2)_{\frac{1}{6}}$ & 1 \\
$U_{i}^{c}$ & $(\overline{3}, 1)_{-\frac{2}{3}}$ & 1 \\
$D_{i}^{c}$ & $(\overline{3}, 1)_{\frac{1}{3}}$ & 1 \\
$E_{j}^{c}$ & $(1,1)_{1}$ & 1 \\
$L_{j}$ & $(1,2)_{-\frac{1}{2}}$ & 1 \\
$H_{u}$ & $(1,2)_{\frac{1}{2}}$ & 0 \\
$R_{d}$ & $(1,2)_{-\frac{1}{2}}$ & 2 \\
$H_{d}$ & $(1,2)_{-\frac{1}{2}}$ & 0 \\
$R_{u}$ & $(1,2)_{\frac{1}{2}}$ & 2 \\
$\Phi_{\tilde{W}}$ & $(1,1)_{0}$ & 0 \\
$\Phi_{\tilde{B}}$ & $(1,3)_{0}$ & 0 \\
$\Phi_{\tilde{g}}$ & $(8,1)_{0}$ & 0
\end{tabular}

Table 3.1: R-charge assignment for the chiral supermultiplets in the MRSSM.

An alternative to enlarging the Higgs sector was proposed in [75]. In this model the Higgs sector contain just two Higgs doublets $H_{u}$, and $R_{d} \cdot R_{d}$ has $R=2$ to allow a $\mu$ term in the superpotential, and it is also necessary to cancel the $H_{u}$ anomalies. However, the down type fermions acquire mass via the $H_{u}$ vev through the following SUSY breaking Yukawa interaction:

$$
\int d^{4} \theta \frac{X^{\dagger}}{M^{2}}\left(y_{d} Q D^{c}+y_{E} L E^{c}\right)
$$

Therefore, in this scenario order to give mass to the bottom quark it is necessary to consider a low scale SUSY breaking. However, this realisation of an $R$ symmetric model has a more minimal particle content than the MRSSM. In the next chapter we will discuss another possible way to minimize the MRSSM particle content where the down type fermions acquire mass through one of the three sneutrinos. 


\section{Chapter 4}

\section{$R$ symmetry as the lepton number}

In the previous chapter we briefly discussed the state of art in $R$ symmetric model building. Here we will focus on models where the $R$ symmetry is identified with the lepton number. First we will discuss a model where the $U(1)_{R}$ symmetry corresponds to one of the lepton number. We will also present its generalisation to a global lepton number $R$ symmetry.

\subsection{Making the sneutrino a Higgs with a $U(1)_{R}$ lep- ton number}

The particle content of our model consists of the usual particle content of the MSSM to which we add an adjoint chiral superfield $\Phi_{i}$ for each SM gauge group $G_{i}=S U(3)_{C}, S U(2)_{L}, U(1)_{Y}$. This is necessary to give Dirac mass to the gauginos and is the minimal particle content needed to accommodate a $U(1)_{R}$ symmetry in a supersymmetric extension of the Standard Model. In fact, this particle content is more minimal than the minimal R-symmetric supersymmetric extension of the Standard Model (MRSSM) presented in [70], as the latter includes two additional weak doublets in order to give mass to the gauginos as the standard $\mu$ term is forbidden by $R$-symmetry. We therefore refer to our model as the More Minimal RSSM 
(MMRSSM). Table 4.1 shows the MMRSSM superfields and their quantum numbers; the $R$ charge assignments is chosen such that we can use the $R$-symmetry as the lepton number of type $a$, where $a=e, \mu$ or $\tau$. Indeed all the Standard Model particles, except the charged lepton $a^{-}$and the neutrino $\nu_{a}$, carry $R$-charge zero. The situation with the SUSY partners is reversed: the charged slepton and the sneutrino of flavour $a$ do not carry any lepton number while all other have lepton number. This means in particular that a sneutrino vev does not break the lepton number, and this is crucial for making the sneutrino the down type Higgs. The squarks are leptoquarks because they carry both the baryon number and the lepton number $a$. As we will show in a following section, this feature characterizes and distinguishes the phenomenology of the model. Moreover, the higgsinos, the wino, the bino together with their adjoint partners carry $R$-charge \pm 1 ; this means they can mix with the ordinary leptons of flavor $a$. In the MMRSSM the lightest chargino and the lightest neutralino coincide with the charged lepton $a^{ \pm}$and the neutrino $\nu_{a}$.

The up-type Higgs $H_{u}$ has $R$ charge 0 , and it acquires a vev. Instead, $R_{d}$ is an inert doublet ${ }^{1}$, which is introduced to cancel the $H_{u}$ anomalies, and to give mass to the higgsinos. It is the sneutrino of flavor $a$ that acquires a vev and gives mass to the down-type fermions.

With this particle content the MMRSSM superpotential is then:

$$
W=\mathbf{y}_{u} U^{c} Q H_{u}-\mathbf{y}_{d} D^{c} Q L_{a}-y_{b} E_{b}^{c} L_{b} L_{a}-y_{c} E_{c}^{c} L_{c} L_{a}+\mu H_{u} R_{d} .
$$

where $\mathbf{y}_{u}$ and $\mathbf{y}_{d}$ are $3 \times 3$ matrices in family space, while $a, b, c=e, \mu$ and $\tau$.

As usual, the up-type fermions acquire mass through $H_{u}$, while the down type Yukawa couplings involve the leptonic superfield $L_{a}$, which then plays the role of the down-type Higgs. However, it is important to note that the superpotential in equation (4.1) does not contain the Yukawa coupling for the lepton of flavor $a$ as

\footnotetext{
${ }^{1}$ This is a common feature of our model with the one Higgs doublet, SOHDM, model introduced in [75].
} 


\begin{tabular}{l|c|l} 
SuperField & $\left(S U(3)_{c}, S U(2)_{L}\right)_{U(1)_{Y}}$ & $U(1)_{R}$ \\
\hline$Q_{i}$ & $(3,2)_{\frac{1}{6}}$ & 1 \\
$U_{i}^{c}$ & $(\overline{3}, 1)_{-\frac{2}{3}}$ & 1 \\
$D_{i}^{c}$ & $(\overline{3}, 1)_{\frac{1}{3}}$ & 1 \\
$E_{a}^{c}$ & $(1,1)_{1}$ & 2 \\
$L_{a}$ & $(1,2)_{-\frac{1}{2}}$ & 0 \\
$E_{b, c}^{c}$ & $(1,1)_{1}$ & 1 \\
$L_{b, c}$ & $(1,2)_{-\frac{1}{2}}$ & 1 \\
$H_{u}$ & $(1,2)_{\frac{1}{2}}$ & 0 \\
$R_{d}$ & $(1,2)_{-\frac{1}{2}}$ & 2 \\
$\Phi_{\tilde{W}}$ & $(1,1)_{0}$ & 0 \\
$\Phi_{\tilde{B}}$ & $(1,3)_{0}$ & 0 \\
$\Phi_{\tilde{g}}$ & $(8,1)_{0}$ & 0
\end{tabular}

Table 4.1: R-charge assignment for the chiral supermultiplets in our model. The subscript $a$ denote the flavour of the lepton superfield that plays the role of the down-type Higgs. The subscrit $b, c$ represent the remaining two flavours of leptons. 
the term $L_{a} L_{a} E_{a}^{c}$ is null, while the term $R_{d} L_{a} E_{a}^{c}$ is forbidden by the $R$-symmetry. Therefore, this coupling needs to be generated in the SUSY breaking sector as we will discuss in a following section. The down-type Yukawa couplings of equation (4.1) violate the conventional $R_{p}$ parity as well as the standard lepton number. Indeed, here these couplings correspond to the trilinear $R_{p}$ violating coupling $\lambda_{i j j} L_{i} L_{j} E_{j}^{c}$, and $\lambda_{i j j}^{\prime} L_{i} Q_{j} D_{j}^{c}$ often discussed in the literature [78]. These couplings have very stringent bounds in conventional $R$-parity breaking models that come from the Majorana neutrino masses they induce. In our model however, there is a conserved lepton number which forbids such masses. In fact in the limit of massless neutrinos, we impose three separate lepton numbers, one for each flavour: $U(1)_{R_{a}}$ which is the $R$-symmetry as well as $U(1)_{b}$ and $U(1)_{c}$ which are not $R$ symmetries. As a consequence, the bounds on those coupling are in the MMRSSM much less stringent than in conventional $R$ parity violating models, and come mainly from electroweak precision measurements. This, as we will see, has interesting phenomenological consequences.

The inert doublet $R_{d}$ does not interact with the SM fermions as the trilinear couplings $D^{c} Q R_{d}$, and $E^{c} L R_{d}$ are forbidden by the $R$-symmetry. As we have already commented, $R_{d}$ is necessary to give mass to the higgsinos. Indeed, a bilinear term $H_{u} L_{a}$ is forbidden by the $R$-symmetry, and the higgsinos acquire mass through the $R$-symmetric $\mu$ term $H_{u} R_{d}$.

Finally, the soft supersymmetry breaking terms allowed by both gauge symmetries and by the $R$-symmetry are:

$$
\mathcal{L}_{\text {soft }}=\mathcal{L}_{\text {mass }}^{f}+\mathcal{L}_{\text {mass }}^{s}-B_{\mu}\left(H_{u} \tilde{l}_{a}+c c\right)
$$

where the gaugino masses are given by:

$$
\mathcal{L}_{\text {mass }}^{f}=M_{\tilde{B}} \tilde{B} \psi_{\tilde{B}}+M_{\tilde{W}} \tilde{W} \psi_{\tilde{W}}+M_{\tilde{g}} \tilde{g} \psi_{\tilde{g}}
$$


and the soft scalar masses by:

$$
\begin{aligned}
\mathcal{L}_{\text {mass }}^{s} & =m_{\tilde{q}}^{2} \tilde{q}^{\dagger} \tilde{q}+m_{\tilde{l}}^{2} \tilde{l}^{\dagger} \tilde{l}+m_{\tilde{u}^{c}}^{2} \tilde{u}^{c^{\dagger}} \tilde{u}^{c}+m_{\tilde{d}^{c}}^{2} \tilde{d}^{\dagger} \tilde{d}^{c} \\
& +m_{e}^{2} \tilde{e}^{c^{\dagger}} \tilde{e}^{c}+m_{H_{u}}^{2} H_{u}^{\dagger} H_{u}+m_{R_{d}}^{2} R_{d}^{\dagger} R_{d}+m_{\Phi_{\tilde{B}}}^{2} \Phi_{\tilde{B}}^{\dagger} \Phi_{\tilde{B}}+ \\
& +m_{\Phi_{\tilde{W}}}^{2} \Phi_{\tilde{W}}^{\dagger} \Phi_{\tilde{W}}+m_{\Phi_{\tilde{g}}}^{2} \Phi_{\tilde{g}}^{\dagger} \Phi_{\tilde{g}}+M_{\Phi_{\tilde{B}}}^{2}\left(\Phi_{\tilde{B}}^{2}+c c\right)+M_{\Phi_{\tilde{W}}}^{2}\left(\Phi_{\tilde{W}}^{2}+c c\right)+M_{\Phi_{\tilde{g}}}^{2}\left(\Phi_{\tilde{g}}^{2}+c c\right) .
\end{aligned}
$$

We notice that equation (4.2) contains a $B$-term that mixes the $\tilde{\nu}_{a}$ sneutrino with $H_{u}$, but not a mixing term for $r_{d}$. This ensures that $r_{d}$ will not get a vev as long it does not acquire a negative mass while the sneutrino will. Moreover, we note that the soft SUSY lagrangian of equation (4.2) does not contain scalar trilinear coupling $A_{i j k}$ nor Majorana mass terms for the gauginos. Finally, as we have already anticipated, the SUSY breaking lagrangian should contain the Yukawa coupling $y_{a} \tilde{h}_{u}^{\dagger} e_{a}^{c} l_{a}$. This term needs to come from the mechanism of SUSY breaking mediation and we will discuss it's origin in section 4.4

\subsubsection{Electroweak symmetry breaking}

In the present section we will study how electroweak symmetry breaking is realized in our model. Such an analysis was also done for a quite general model in [81]. The part of the potential that is relevant for electroweak symmetry breaking contains only $h_{u}^{0}$, $\tilde{\nu}_{a}$ as well as the adjoint scalars $\tilde{\phi}_{\tilde{B}}$, and $\tilde{\phi}_{\tilde{W}}$ as they can acquire a non-zero vev. All other fields do not get a vev and are set to 0 in what follows. The potential consists of three terms:

$$
V_{E W}=V_{D}+V_{F}+V_{\text {soft }}
$$

The first is the contribution from the $S U(2)_{L}$ and $U(1)_{Y}$ D-term and is given by:

$V_{D}=\frac{1}{2}\left(\sqrt{2} M_{\tilde{B}}^{2}\left(\tilde{\phi}_{\tilde{B}}+\tilde{\phi}_{\tilde{B}}^{\dagger}\right)+\frac{g^{\prime}}{2}\left(\left|H_{u}^{0}\right|^{2}-\left|\tilde{\nu}_{a}\right|^{2}\right)\right)^{2}+\frac{1}{2}\left(\sqrt{2} M_{\tilde{W}}^{2}\left(\tilde{\phi}_{\tilde{W}}^{0}+\tilde{\phi}_{\tilde{W}}^{0 *}\right)+\frac{g}{2}\left(\left|H_{u}^{0}\right|^{2}-\left|\tilde{\nu}_{a}\right|^{2}\right)\right)^{2}$, 
The second contribution comes, instead, from the superpotential, and it only contains a mass term for the up-type Higgs:

$$
V_{F}=\mu^{2}\left|H_{u}^{0}\right|^{2}
$$

Finally, the third contribution contains the following soft SUSY breaking terms:

$$
\begin{gathered}
V_{\text {soft }}=m_{\tilde{\phi}_{\bar{B}}}^{2} \tilde{\phi}_{\tilde{B}^{\prime}}^{\dagger} \tilde{\phi}_{\tilde{B}}+m_{\tilde{\phi}_{\bar{W}}}^{2} \tilde{\phi}_{\bar{W}^{\prime}}^{\dagger} \tilde{\phi}_{\tilde{W}}+M_{\tilde{\phi}_{\bar{B}}}^{2}\left(\tilde{\phi}_{\tilde{B}}^{2}+c c\right)+M_{\tilde{\phi}_{\bar{W}}}^{2}\left(\tilde{\phi}_{\tilde{W}}^{2}+c c\right)+ \\
m_{H_{u}}^{2}\left|H_{u}^{0}\right|^{2}+m_{L_{a}}^{2}\left|\tilde{\nu}_{a}^{2}\right|-B_{\mu}\left(H_{u}^{0} \tilde{\nu}_{a}+h . c .\right) .
\end{gathered}
$$

The scalar potential is then:

$$
\begin{aligned}
V_{E W}= & \left(\mu^{2}+m_{H_{u}}^{2}\right)\left|H_{u}^{0}\right|^{2}+m_{\tilde{\nu}_{a}}^{2}\left|\tilde{\nu}_{a}\right|^{2}-B_{\mu}\left(H_{u}^{0} \tilde{\nu}_{a}+h . c .\right)+\frac{g^{\prime}+g}{8}\left(\left|H_{u}\right|^{0}-\left|\tilde{\nu}_{a}\right|^{2}\right)^{2}+ \\
& +\frac{1}{2}\left(m_{\tilde{\phi}_{\tilde{B}}}^{2}+M_{\tilde{\phi}_{\tilde{B}}}^{2}+4 M_{\tilde{B}}^{2}\right) \tilde{\phi}_{\tilde{B}}^{R 2}+g^{\prime} M_{\tilde{B}}^{2} \tilde{\phi}_{\tilde{B}}^{R}\left(\left|H_{u}\right|^{0}-\left|\tilde{\nu}_{a}\right|^{2}\right)+g M_{\tilde{W}}^{2} \tilde{\phi}_{\tilde{W}}^{R}\left(\left|H_{u}^{0}\right|^{2}-\left|\tilde{\nu}_{a}\right|^{2}\right) .
\end{aligned}
$$

with $\tilde{\phi}_{i}^{R}$ denoting the real part of $\tilde{\phi}_{i}$.

As we have already noticed, in gauge mediation models, the adjoint scalars are the heaviest particle of the spectrum [68] and can be integrated out of the potential. This has two effects: first it lowers the Higgs quartic and second it shift the mass of the $Z$ boson, creating a contribution to the $\rho$ parameter:

$$
\Delta \rho=\frac{v^{2}}{M_{\Phi_{W}^{R}}^{4}} g^{2} M_{\tilde{W}} \cos (2 \beta)
$$

where $M_{\Phi_{W}^{R}}^{2}=m_{\Phi_{\tilde{W}}}^{2}+M_{\Phi_{\tilde{W}}}^{2}+4 M_{\tilde{W}}^{2}$ is the mass of the real part of the $S U(2)$ adjoint scalar and $\tan \beta$ is the ratio of the vev of the up-type Higgs and the vev of the sneutrino: $\tan \beta=v_{u} / v_{a}$. With $M_{\Phi_{W}^{R}}$ larger than a few $\mathrm{TeV}$, the above contribution to $\rho$ is within the experimental bound, and we can neglect the correction to the Higgs potential and minimize the following potential:

$$
V_{E W}=\left(\mu^{2}+m_{H_{u}}^{2}\right)\left|H_{u}^{0}\right|^{2}+m_{\tilde{\nu}_{a}}^{2}\left|\tilde{\nu}_{a}\right|^{2}-B_{\mu}\left(H_{u}^{0} \tilde{\nu}_{a}+h . c .\right)+\frac{g^{2}+g^{\prime 2}}{8}\left(\left|H_{u}^{0}\right|^{2}-\left|\tilde{\nu}_{a}\right|^{2}\right)^{2} .
$$


This is exactly the scalar potential of the MSSM with $H_{d}^{0} \rightarrow \tilde{\nu}_{a}$, except that here we do not have the $\mu$ contribution to the sneutrino $\tilde{\nu}_{a}$ mass, as the $R$ invariant $\mu$ term contains only $H_{u}$. Therefore, in order for the potential to be bounded from below the quadratic part should be positive along the $D$ flat directions:

$$
2 B_{\mu}<\mu^{2}+m_{H_{u}}^{2}+m_{\tilde{\nu}_{a}}^{2}
$$

Furthermore, the condition for electroweak symmetry breaking is:

$$
\begin{gathered}
B_{\mu}^{2}>\left(\mu^{2}+m_{H_{u}}^{2}\right) m_{\tilde{\nu}_{a}}^{2} . \\
\sin \beta=\frac{2 B_{\mu}}{m_{H_{u}}^{2}+\mu^{2}+m_{L_{a}}^{2}}, \\
M_{Z}^{2}=\frac{\left|\mu^{2}+m_{H_{u}}^{2}-m_{L_{a}}^{2}\right|}{\sqrt{1-\sin ^{2} \beta}}-m_{H_{u}}^{2}-m_{L_{a}}^{2}-\mu^{2} .
\end{gathered}
$$

The spectrum of the Higgs sector of the model contains the usual CP odd neutral particle $A^{0}$, the two CP even $H^{0}, h^{0}$, and the charged Higgs. Their masses are:

$$
\begin{aligned}
m_{A^{0}}^{2} & =\frac{2 b}{\sin 2 \beta}=m_{H_{u}}^{2}+\mu^{2}+m_{L_{a}}^{2}, \\
m_{H^{ \pm}}^{2} & =m_{A^{0}}^{2}+m_{W}^{2}, \\
m_{h^{0}, H^{0}}^{2} & =\frac{1}{2}\left(m_{A^{0}}^{2}+m_{Z^{0}}^{2} \mp+\sqrt{\left(m_{A^{0}}^{2}-m_{Z^{0}}^{2}\right)^{2}+m_{A^{0}}^{2} m_{Z^{0}}^{2} \sin ^{2} 2 \beta} .\right.
\end{aligned}
$$

This is identical to the case of the MSSM and we therefore, we inherit also the MSSM little hierarchy problem. In a $R$-symmetric model this problem could be even more severe. The $R$-symmetry forbids the left/right stop mixing, and this reduces the contribution of the stop radiative corrections to the SM Higgs mass. Indeed, the full one loop contribution of the stop sector to the Higgs mass is [57]:

$$
\begin{aligned}
\delta m_{h^{0}}^{2} & =\frac{3}{4 \pi^{2}} \sin ^{2} \beta y_{t}^{2}\left[m_{t}^{2} \ln \left(\frac{m_{\tilde{t}_{1}} m_{\tilde{t}_{2}}}{m_{t}^{2}}\right)+c_{\tilde{t}}^{2} s_{\tilde{t}}^{2}\left(m_{\tilde{t}_{2}}^{2}-m_{\tilde{t}_{1}}^{2}\right) \ln \left(\frac{m_{\tilde{t}_{1}}^{2}}{m_{\tilde{t}_{2}}^{2}}\right)+\right. \\
& \left.+c_{\tilde{t}}^{4} s_{\tilde{t}}^{4}\left(\left(m_{\tilde{t}_{2}}^{2}-m_{\tilde{t}_{1}}^{2}\right)^{2}-\frac{1}{2}\left(m_{\tilde{t}_{2}}^{4}-m_{\tilde{t}_{1}}^{4}\right) \ln \left(\frac{m_{\tilde{t}_{1}}^{2}}{m_{\tilde{t}_{2}}^{2}}\right)\right) / m_{t}^{2}\right]
\end{aligned}
$$


where $c_{\tilde{t}}$, and $s_{\tilde{t}}$ are the cosine and the sine of the stop mixing angle, and $\tilde{t}_{1}, \tilde{t}_{2}$ the mass eigenstate. From eq.(4.20) we see how the absence of left/right mixing considerably reduces the radiative contribution from the stop sector forcing the mass of the stop to increase in order to make the Higgs sufficiently heavy.

On the other hand, the supersoft SUSY breaking mechanism ameliorates the fine tuning problem, because now the radiative contribution to $M_{H_{u}}^{2}$ is:

$$
\Delta M_{H_{u}}^{2}=\frac{3 y_{t} m_{\tilde{t}}^{2}}{4 \pi^{2}} \ln \frac{m_{\tilde{t}}}{\Lambda}
$$

where the cutoff scale $\Lambda$ is the mass of the real adjoint scalars, and not the messenger scale as in the typical gauge mediation scenarios.

As in the MSSM, one might wonder how to increase the Higgs quartic coupling, and reduce in this way the fine tuning. The only $R$ symmetric dimension five operator that gives a contribution to the Higgs quartic coupling is:

$$
\int \frac{d^{2} \theta}{M}\left(H_{u} H_{d}\right)\left(H_{u} L_{a}\right)
$$

A possible way to generate this operator is to introduce a singlets which couples to the Higgs superfields in the following way:

$$
m_{S} S \bar{S}+k_{1} H_{u} H_{d} S+k_{2} H_{u} L_{a} \bar{S}
$$

This is a possible solution to the little hierarchy problem in our model inspired by the NMSSM. Alternatively, if we consider a very low SUSY breaking scale $\frac{F}{M^{2}} \sim 1$ we might be able to increase the Higgs quartic coupling through the following operator:

$$
\int d^{4} \theta \frac{X^{\dagger} X}{M^{4}}\left(H_{u}^{\dagger} H_{u}\right)^{2}
$$

We plan to explore in more detail the fine tuning problems of the model in future work. 


\subsubsection{Lepton mixing}

In the MMRSSM all the sparticles are $a$ leptons, except for the sneutrino and the slepton of flavour $a$. In particular, the new fermions (gauginos, adjoints, higgsinos), and the neutrino $\nu_{a}$ as well as the charged lepton $a^{-}$carry $R$ charge \pm 1 , and therefore they can all mix.

In the gauge eigenstate basis with $\Psi_{+}=\left(\tilde{W}^{+}, \psi_{\tilde{W}}^{+}, \tilde{H}_{u}^{+}, a^{c}\right)$ and $\Psi_{-}=$ $\left(\tilde{W}^{-}, \psi_{\bar{W}}^{-}, \tilde{R}_{d}^{-}, a^{-}\right)$the chargino mass term is given by:

$$
\mathcal{L}_{C}=\Psi_{-}^{T} M_{C} \Psi_{+}
$$

where:

$$
M_{C}=\left(\begin{array}{cccc}
0 & M_{\tilde{W}} & -\frac{g v_{u}}{\sqrt{2}} & 0 \\
M_{\tilde{W}} & 0 & 0 & 0 \\
0 & 0 & \mu & 0 \\
-\frac{g v_{a}}{\sqrt{2}} & 0 & 0 & m_{a}
\end{array}\right) .
$$

The smallest eigenvalue corresponds to the mass of the charged lepton $a^{-}$and is give by $m_{a}$ to first order in $v_{a}^{2} / M_{\bar{W}}^{2}$. The left-handed component of the charged lepton $a^{-}$ mixes with the charged components of the adjoint triplet $\psi_{\tilde{W}}$, that is:

$$
a^{\prime-}=\cos \phi a^{-}+\sin \phi \psi_{\tilde{W}}^{-}
$$

where the mixing angles are:

$$
\begin{aligned}
& \cos \phi=-\frac{\sqrt{2} M_{\tilde{W}}}{\sqrt{\left(2 M_{\tilde{W}}^{2}+g^{2} v_{a}^{2}\right)}} \sim-1+g^{2} \frac{v_{a}^{2}}{M_{\tilde{W}}^{2}}+O\left(\frac{v_{a}^{2}}{M_{\tilde{W}}^{2}}\right), \\
& \sin \phi=\frac{g v_{a}}{\sqrt{\left(2 M_{\tilde{W}}^{2}+g^{2} v_{a}^{2}\right)}} \sim \frac{v_{a}}{M_{\tilde{W}}}+O\left(\frac{v_{a}^{2}}{M_{\tilde{W}}^{2}}\right) .
\end{aligned}
$$

In the same way the neutrino $\nu_{a}$ corresponds to the lightest neutralino. In the gaugeeigenstates basis $\Psi_{1}^{0}=\left(\tilde{B}, \tilde{W}^{0}, \tilde{H}_{d}^{0}\right)$, and $\Psi_{-1}^{0}=\left(\tilde{H}_{u}^{0}, \nu_{a}, \psi_{\tilde{B}}^{0}, \psi_{\tilde{W}}^{0}\right)$ the neutralinos mass 
term has the form:

$$
\mathcal{L}_{N}=-\frac{1}{2}\left(\Psi_{-1}^{0}\right)^{T} M_{N} \Psi_{1}^{0}+c . c .
$$

where the mass matrix is:

$$
M_{N}=\left(\begin{array}{ccc}
\frac{g^{\prime} v_{u}}{\sqrt{2}} & -\frac{g v_{u}}{\sqrt{2}} & -\mu \\
\frac{g^{\prime} v_{a}}{\sqrt{2}} & -\frac{g v_{a}}{\sqrt{2}} & 0 \\
M_{\tilde{B}} & 0 & 0 \\
0 & M_{\tilde{W}} & 0
\end{array}\right)
$$

Then, the physical neutrino corresponds to the following mixture:

$$
\nu_{a}^{\prime}=c_{\nu} \nu_{a}+c_{\tilde{B}} \psi_{\tilde{B}}+c_{\tilde{W}} \psi_{\tilde{W}}
$$

where the mixing angle:

$$
\begin{aligned}
c_{\nu} & =-\frac{1}{\sqrt{\frac{1}{2}\left(\frac{g v_{a}}{M_{\tilde{W}}}\right)^{2}+\frac{1}{2}\left(\frac{g^{\prime} v_{a}}{M_{\tilde{B}}}\right)^{2}+1}}, \\
c_{\tilde{B}} & =-\frac{g^{\prime} v_{a}}{\sqrt{2} M_{\tilde{B}} \sqrt{\frac{1}{2}\left(\frac{g v_{a}}{M_{\tilde{W}}}\right)^{2}+\frac{1}{2}\left(\frac{g^{\prime} v_{a}}{M_{\tilde{B}}}\right)^{2}+1}}, \\
c_{\tilde{W}} & =\frac{g v_{a}}{\sqrt{2} M_{\tilde{W}} \sqrt{\frac{1}{2}\left(\frac{g v_{a}}{M_{\tilde{W}}}\right)^{2}+\frac{1}{2}\left(\frac{g^{\prime} v_{a}}{M_{\tilde{B}}}\right)^{2}+1}}
\end{aligned}
$$

\subsection{Constraints from electroweak precision mea-}

\section{surement}

In the present section we will discuss constraints on our models from electroweak precision measurements (EWPM) and we will show that the MMRSSM parameter space compatible with the EWPM is large. First, we will present bounds on the sneutrino vev coming from lepton mixing and subsequentely we will discuss the EWPM 

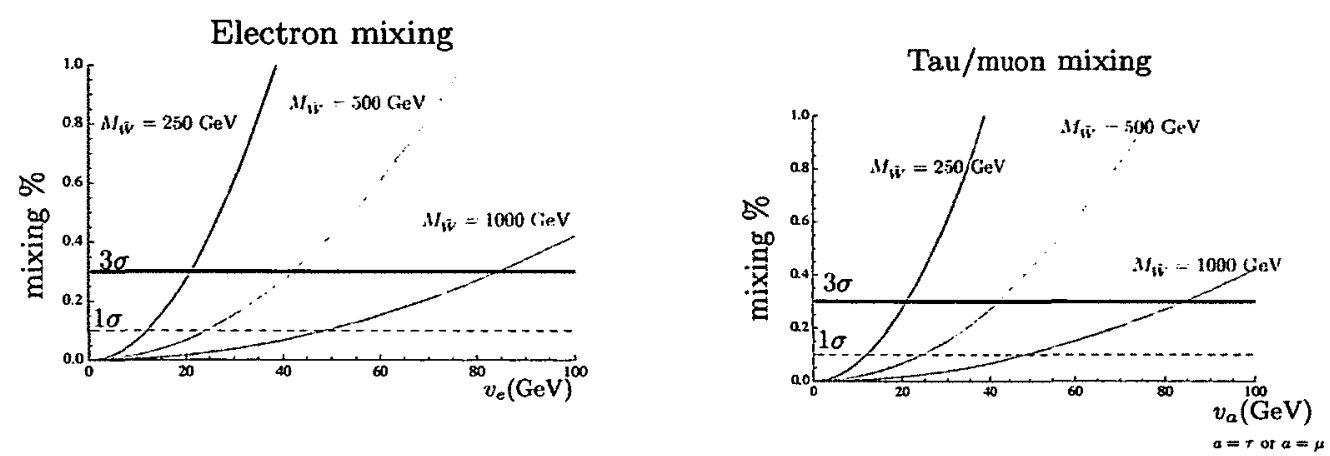

Figure 4.1: The lepton mixing with the triplet (right electron and left for the muon or for the tau) taking into account different values of the wino mass $\left(M_{\bar{W}}=250 \mathrm{GeV}\right.$, $M_{\tilde{W}}=500 \mathrm{GeV}$, and $M_{\tilde{W}}=1000 \mathrm{GeV}$ ). The blue horizontal line represents the $1 \sigma$ threshold, and the one the $3 \sigma$ threshold.

limits on the down type Yukawa couplings that then translate in upper bounds on the sneutrino vev.

As we showed in the previous section, the MMRSSM the charged lepton $a^{-}$, and the neutrino $\nu_{a}$ mix with the adjoint fermions as they both carry $R$ charge \pm 1 . The mixing changes the coupling of the lepton of flavour $a$ to the vector bosons and this will lead to deviations in predictions for EWPM. It is therefore essential to check under which conditions they are compatible with observations.

The mixing of the charged lepton of flavour $a$ to the triplet leads the following modifications to its coupling to the $Z$ boson:

$$
\mathcal{L}_{\mathrm{NC}}=\frac{g}{2 \cos \theta_{W}} \bar{\psi}_{a} \gamma^{\mu}\left(g_{V \mathrm{SM}}^{a}+\delta g_{V}^{a}-\left(g_{A \mathrm{SM}}^{a}+\delta g_{A}^{a}\right) \gamma^{5}\right) \psi_{a} Z_{\mu}
$$

where $\psi_{a}$ is the Dirac 4-component spinors for the charged lepton of flavour $a$, while the corrections to the Standard Model coupling can be expressed in terms of the mixing angles of eq.(4.27):

$$
\delta g_{V}^{a}=\delta g_{A}^{a}=-\frac{\sin ^{2} \phi}{2} .
$$

We can compare these corrections to the measured values of $g_{V}^{a}$ and $g_{A}^{a}$ [82] shown in table 4.2. If we impose that $\delta g_{V}^{a}$, and $\delta g_{A}^{a}$ be within the experimental error, we 


\begin{tabular}{|c||c||c|}
\hline Lepton & $g_{V}^{l}$ & $g_{A}^{l}$ \\
\hline$e$ & $-0.03817 \pm 0.00047$ & $-0.50111 \pm 0.00035$ \\
$\mu$ & $-0.0367 \pm 0.0023$ & $-0.50120 \pm 0.00054$ \\
$\tau$ & $-0.0366 \pm 0.0010$ & $-0.50204 \pm 0.00064$ \\
\hline
\end{tabular}

Table 4.2: Effective vector-axial lepton couplings.

obtain that a mixing smaller than $0.07 \%$ is tolerated at $1 \sigma$ level by EWPM when $a=e$. For $a=\mu$, and $a=\tau$, the limit is $0.1 \%$. Inserting eq.(4.27) in eq.(4.35) we obtain bounds on the sneutrino VEV which are shown in fig. 4.1. For winos at the electroweak scale the region allowed by the experimental data is a fairly high $\tan \beta$ region $(\tan \beta>11)$ at $1 \sigma$ level. However, it is possible to enlarge the parameter space by considering heavier gauginos, for example $M_{\tilde{W}}=1 \mathrm{TeV}$ requires only $\tan \beta>2$. Therefore, the MMRSSM tends to favor a scenario with fairly heavy gauginos .

Since only one of the flavour mixes with the triplet, lepton universality is broken in our model. Charged current universality is verified experimentally to the $0.2 \%$ level for both $e-\mu$, and $\mu-\tau[83,84]$, but we find that we do not obtain stronger bounds from this fact than those derived from the $Z$ coupling. This is shown in fig 4.2 where we plotted $\frac{g_{\tau}}{g_{\mu}}-1$, taking $a=\tau$, namely:

$$
\frac{g_{\tau}}{g_{\mu}}=\cos \phi c_{\nu}+\sqrt{2} \sin \phi c_{\psi_{\tilde{W}}}
$$

In the MMRSSM the down-type Yukawa couplings give extra tree level contributions to electroweak observables which put constraints one those couplings, and therefore put a lower bound on the sneutrino vev. As we have already noticed in the previous section, the MMRSSM down-type Yukawa couplings have the same form as standard $R_{p}$ violating trilinear couplings. Indeed, the lepton Yukawa couplings correspond in the standard notation to the $\lambda_{i j k} L_{i} L_{j} E_{k}^{c}$ couplings, while the down type quark Yukawa couplings correspond to $\lambda_{i j k}^{\prime} L_{i} Q_{j} D_{k}^{c}$. Therefore, these extra tree level 


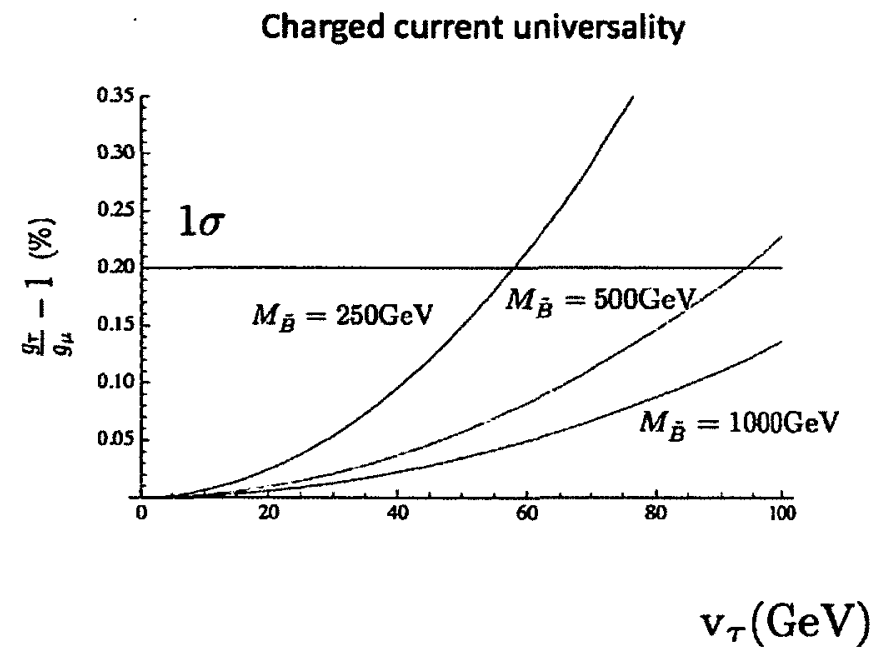

Figure 4.2: The violation of leptonic universality in the charged current interaction $1-\frac{g_{\tau}}{g_{\mu}}$ assuming the the mixed lepton is the $\tau$. We considered values of $M_{\tilde{W}}=250$ $\mathrm{GeV}^{g_{\mu}}, M_{\tilde{W}}=500 \mathrm{GeV}$, and $M_{\tilde{W}}=1000 \mathrm{GeV}$. The horizontal line represents the $0.3 \%$ threshold, and the one the $3 \sigma$ threshold.

contribution to the electroweak observables are the same as in standard $R_{p}$ violating models and we can use result from the literature on those models (see [78] for a review) to put bounds on the Yukawa couplings of our model.

The strongest bound when $a=e$ or $a=\mu$ comes from the tau Yukawa coupling $L_{e} L_{\tau} E_{e}^{c}$ ( or $L_{\tau} L_{\mu} E_{\mu}^{c}$ ) These operators lead to an additional contribution to the leptonic tau decays via $\tilde{\tau}_{R}$ exchange. This affects the ratio $R_{\tau \mu}$, defined as:

$$
R_{\tau \mu}=\frac{\Gamma(\tau \rightarrow \mu \nu \nu)}{\Gamma(\tau \rightarrow e \nu \nu)}
$$

and leads to the following bound:

$$
y_{\tau}<0.07\left(\frac{100 \mathrm{GeV}}{m_{\tilde{\tau}^{c}}}\right)^{2} .
$$

This bound implies a lower limit for the sneutrino vev $v_{a}>15 \mathrm{GeV}$ both for $a=e$, and for $a=\mu$. We see that this would exclude the region of the parameter space with gauginos with a mass around the electroweak scale. Therefore, the MMRSSM 
spectrum is characterised by fairly heavy gauginos or in another words the very high $\tan \beta$ region in the MMRSS is excluded by the experimental constraints on the Yukawa coupling.

When $a=\tau$ the strongest bound on the sneutrino vev comes from the bottom Yukawa coupling. The trilinear coupling $L_{a} Q b^{c}$ leads to an additional contribution to at loop level to the partial width of the $Z$ to $\tau$. The comparison with experiment gives the following bound:

$$
\left|y_{b}\right|<0.58\left(\frac{m_{\tilde{b}_{R}}}{100 \mathrm{GeV}}\right)^{2}
$$

Therefore, the MMRSSM parameter space for $a=\tau$ is less constrained, and in particular it contains also a very $\tan \beta$ region.

In the standard $R_{p}$ violating scenario, the EWPM bounds are subleading compared to the bounds that come from the generation of Majorana mass for neutrinos. If we consider for example $\lambda_{a 33}^{\prime}=y_{b}^{a}$, that is the bottom Yukawa coupling in our model, we see that the constraints on the neutrino mass require: $\lambda_{a 33}^{\prime}>10^{-6}$, while in our case the same coupling can be several orders of magnitude bigger: $y_{b}^{\tau}>0.58$. We will investigate the phenomenological consequences of this in section 5 .

Standard $R_{p}$ violating trilinear couplings are also constrained by cosmological bounds and these constraints can be quite stringent. For example, the requirement that an existing baryon asymmetry is not erased before the electroweak transition typically implies [85] $\lambda, \lambda^{\prime}<10^{-7}$. These constraints do not apply to our case, as the model preserves the baryonic number as well as lepton number. However, as we will see in the following section, the MMRSSM requires a very low re-heating temperature and would require a different baryogenesis mechanism. 


\section{3 $R$-symmetry breaking}

$R$-symmetry is not an exact symmetry because it is broken (at least) by the gravitino mass term that is necessary to cancel the cosmological constant. This breaking is then communicated to the visible sector, through anomaly mediation if nothing else. Therefore, we need to take into account the following additional anomaly-mediated, $R$-symmetry violating soft terms [57]:

$$
\begin{aligned}
\mathcal{L}_{A M}= & m_{B} \tilde{B} \tilde{B}+m_{\tilde{W}} \operatorname{tr}(\tilde{W} \tilde{W})+m_{g} \operatorname{tr}(\tilde{g} \tilde{g})+ \\
& A_{b c}^{l} \tilde{\ell}_{a} \tilde{\ell}_{b} e_{c}^{c}+A_{j k}^{D} \tilde{\ell}_{a} \tilde{q}_{j} \tilde{d}_{k}^{c}+h_{u} \tilde{q} A^{U} \tilde{u}^{c}
\end{aligned}
$$

where:

$$
\begin{aligned}
& M_{\lambda_{i}}=\beta_{i} \frac{\alpha_{i}}{4 \pi} m_{\frac{3}{2}} \\
& A_{j k}=-\beta_{y_{j k}} m_{\frac{3}{2}}
\end{aligned}
$$

where $m_{\frac{3}{2}}=\frac{\Lambda^{2}}{M_{P}}$ is the gravitino mass, and $\Lambda \sim \sqrt{D^{\prime}}$ indicates the SUSY breaking scale. Therefore, the gauginos are not pure Dirac fermions, but pseudo Dirac. For relatively low SUSY breaking scale $\Lambda$ these contributions will be subdominant compared to the $R$-symmetric SUSY breaking terms in equation (4.2) and will not have important phenomenological consequences. One important exception is that they will generate neutrino masses that can be above the present bound.

\subsubsection{Neutrino masses}

The SUSY breaking term of equation (4.41) also break the $U(1)_{R}$ symmetry and will inevitably generate a Majorana mass term for the neutrino of flavour $a$, and this will translate to a limit on the SUSY breaking scale.

At tree level the neutrino remains massless. Indeed, even after introducing the Majorana masses $M_{\lambda_{i}}$ for the gauginos in the neutralino mass matrix of equation 


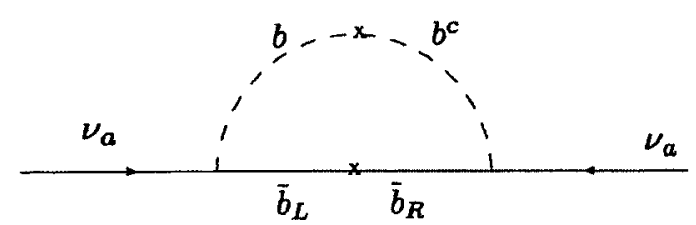

Figure 4.3: Majorana mass term for the neutrino $\nu_{a}$ generated at one loop from anomaly mediation ( by gaugino Majorana mass on the right, and left/right mixing on the left).

(4.29) the smallest eigenvalue is still zero. At one loop a Majorana mass term for $\nu_{a}$ is induced by the diagrams in fig.4.3. The contribution coming from the insertion of an $A$ term is given parametrically by (see [78] for the full expression):

$$
M_{\nu_{a}} \sim 3\left(\frac{1}{16 \pi^{2}}\right)^{2}\left(\frac{m_{b}}{m_{\tilde{b}}}\right)^{2} y_{b}^{2} m_{\frac{3}{2}}
$$

where $\tilde{m}_{b}$ is an averaged sbottom mass parameter. The mass contributions in equation (4.43) is suppressed by the Yukawa couplings that assume their maximum values at large $\tan \beta$. For example, when $v_{a} \sim 5 \mathrm{GeV}$ and $m_{\tilde{b}} \sim 200 \mathrm{GeV}$, requiring $M_{\nu_{a}} \lesssim 1$ $\mathrm{eV}$ leads to: $m_{\frac{3}{2}} \lesssim 10 \mathrm{MeV}$ which implies $\Lambda \lesssim 10^{8} \mathrm{GeV}$. The contribution from the diagram with a Majorana gaugino mass insertion is given parametrically in the large $\tan \beta$ limit by:

$$
M_{\nu_{a}} \sim\left(\frac{1}{16 \pi^{2}}\right) \frac{m_{Z}^{2}}{m_{\chi_{0}}^{2}} \frac{M_{\lambda}}{\tan ^{2} \beta}
$$

where $m_{\chi_{0}}$ is the neutralino mass and $M_{\lambda} \sim m_{\frac{3}{2}} /\left(16 \pi^{2}\right)$ is the Majorana gaugino mass insertion. The corresponding bound is then stronger for lower $\tan \beta$. For $v_{a}=100$ $\mathrm{GeV}$ and $m_{\chi_{0}}=1 \mathrm{TeV}$, asking for $M_{\nu_{a}} \lesssim 10 \mathrm{eV}$ leads to $m_{3 / 2} \lesssim 1 \mathrm{MeV}$ which implies $\Lambda \lesssim 3 \times 10^{7} \mathrm{GeV}$.Therefore, the MMRSSM is compatible with the bounds on the neutrino masses, as long as we consider a fairly low SUSY breaking scale like a scenario of gauge mediated SUSY breaking.

Neutrino masses for the other flavour $b, c$ can be introduced through higher dimensional operators of the form:

$$
\int d^{2} \theta \frac{\left(H_{u} L_{b, c}\right)\left(H_{u} L_{b, c}\right)}{M_{f}}
$$


where the scale $M_{f}$ is a flavor scale where the overall lepton number $L_{b}+L_{c}$ is broken.

\section{$4.4 \quad R$-symmetric gauge mediation}

The bounds on neutrino masses from section 4.3.1 require a low SUSY breaking scale. This means that high scale SUSY breaking mechanism such as gravity or anomaly mediated SUSY breaking will not work in our context and R-symmetric gauge mediation is a more natural possibility. $\mathrm{R}$-symmetric gauge mediation was studied in some details in [68] and [80]. One of the main issue is to generate positive masses for the adjoint scalars. This can be achieved with an appropriate choice of couplings between the adjoint superfields and the messengers. In the MMRSSM, the $\mu / B_{\mu}$ problem takes a slightly different form, and the susy breaking mediation mechanism also need to generate the Yukawa coupling for the lepton of flavour $a$ which is not generated in the low energy theory.

\subsection{1 $R$ symmetry, and the $\mu / B_{\mu}$ problem}

In $R$ symmetric models the $\mu / B_{\mu}$ problem is a different problem than in the $\mathrm{MSSM}^{2}$ . Indeed, the $\mu$, and the $B_{\mu}$ terms contain different fields and therefore, they can be generated by separate UV physics. For example, in the MMRSSM the $\mu$ term is $\mu \tilde{H}_{u} \tilde{R}_{d}$, while the $B_{\mu}$ term is $B_{\mu} H_{u} \tilde{l}_{a}$. In the MRSSM, instead, the $\mu$ term has the form $\tilde{R}_{u} \tilde{H}_{d}+\tilde{H}_{u} \tilde{R}_{d}$, while the $B_{\mu}$ term is $B_{\mu} H_{u} H_{d}$ [91] (see also [92] for a model without a $\mu$ term). This facilitate the generation of the $\mu$ term at one loop, and the $B_{\mu}$ term at two loops. However, as we will see below, this is not sufficient to assure the naturalness of the model.

If we assume that SUSY is broken only by the D-term of vector superfield spurion,

\footnotetext{
${ }^{2}$ For a discussion of the $\mu B_{\mu}$ problem in model with Dirac gaugino, but with $R$ symmetry breaking in the Higgs sector see [90]
} 


\begin{tabular}{c|c} 
SuperField & $U(1)_{R}$ \\
\hline$\Phi$ & 0 \\
$\bar{\Phi}$ & 2 \\
$S$ & 0 \\
$\bar{S}$ & 2 \\
$\bar{N}$ & 0 \\
$N$ & 2
\end{tabular}

Table 4.3: R-charge assignement

the effective operators which generate the $\mu$ and $B_{\mu}$ terms are:

$$
\begin{aligned}
& \frac{1}{M^{3}} \int d^{4} \theta\left(W^{\prime \alpha} W_{\alpha}^{\prime}\right)^{\dagger} H_{u} R_{d}, \\
& \frac{1}{M^{6}} \int d^{4} \theta\left(W^{\prime} \alpha W_{\alpha}^{\prime}\right)^{2} H_{u} L_{a}
\end{aligned}
$$

If $M$ is the messenger mass scale, $D^{\prime} \ll M^{2}$ and the $\mu$ term is too small: $\mu \sim \frac{D^{2}}{16 \pi^{2} M^{3}}$, unless $D \sim M^{2}$ or $D \sim 10^{-1} M^{2}$ with the gauginos at the TeV scale. Another possibility is that the denominators of the operator (4.47) are made out of different mass scales, similar to the model of [94] We can for example write a superpotential of the form :

$$
\begin{aligned}
W_{\mu B \mu}^{D} & =M_{\Phi} \Phi_{+} \Phi_{-}+M_{S} \bar{S} S+M_{N} \bar{N} N+ \\
& +S\left(\lambda_{1} R_{d} H_{u}+\lambda \Phi_{+} \Phi_{-}\right)+\lambda_{2} S^{2} N+\tilde{\lambda}_{1} H_{u} L_{a} N
\end{aligned}
$$

where $\Phi_{+,-}$are messenger fields that are singlet under the SM gauge groups, which carry $U(1)^{\prime}$ charge, R-charge 1 and get soft mass terms from the $D^{\prime}$-term.

The other fields, $S, \bar{S}, N, \bar{N}$ are all singlet under the SM gauge groups and have the $R$-charge assignement shown in table 4.3 :

The $\mu$ term is then:

$$
\mu \sim \frac{\lambda \lambda_{1}}{16 \pi^{2}} \frac{D^{\prime 2}}{M_{T} M_{S}^{2}}
$$


where $M_{T}$ is the mass of the messenger scalars $\Phi_{+,-}$. If one then assumes $M_{S} \sim \sqrt{D^{\prime}}$, on can get $\mu$ term at the weak scale or little bit above. The $B_{\mu}$ term needs to involve the superfield $N$ and will be generated at two loops with the same size as $\mu^{2}$. In models with a SUSY breaking spurion with an $F$-term: $X=\theta^{2} F$, the $\mu$ and the $B_{\mu}$ terms could be generated through the following effective operators:

$$
\begin{gathered}
\frac{1}{M} \int d^{4} \theta X^{\dagger} H_{u} R_{d}, \\
\frac{1}{M^{2}} \int d^{4} \theta\left(X^{\dagger} X\right) H_{u} L_{a} .
\end{gathered}
$$

As usual, in order to avoid fine tuning problems, the $\mu$, and the $B_{\mu}$ terms should be of the same order, that is $B_{\mu} \sim \mu^{2}$ This means that $B_{\mu}$ needs to be generated at two loops, while $\mu$ has to be generated at one loop order.

In our model the $\mu$ and the $B_{\mu}$ terms are generated by operators with different fields and this makes it easier to write down a superpotential, that possesses accidental symmetries which forbids the $B_{\mu}$ term at one loop and allow, instead, the generation of the $\mu$ term. But this is not sufficient to guarantee the naturalness of our model as the new couplings of the Higgs with the messenger sector can generate additional soft mass terms $m_{H_{u}}^{2}$ at one loop, which would be larger than the one coming from the $\mu$ term by the square root of a one loop factor. This problem can be addressed by considering a model analogous to the one considered in [94] that does not couple the Higgs superfield directly to the messenger, but use some extra singlet to generate the $\mu$ term. In this case the $\mu$ term arises from an operator of the form:

$$
\int d^{4} \theta \frac{D^{2}\left(X^{\dagger} X\right)}{M^{3}} H_{u} R_{d}
$$

instead of (4.51). The $B_{\mu}$ term also receives contribution from an operator of the form

$$
\int d^{4} \theta \frac{\left(X^{\dagger} X\right) \bar{D}^{2} D^{2}\left(X^{\dagger} X\right)}{M^{6}} H_{u} L_{a}
$$


Notice the scaling of those operators is very similar to the one in (4.47). They can also be generated through a very similar superpotential with one vector-like messenger field $\Phi, \bar{\Phi}$ and two singlet $N, \bar{N}$ and $S, \bar{S}$ :

$$
\begin{aligned}
W_{\mu B \mu} & =M_{\Phi} \bar{\Phi} \Phi+M_{S} \bar{S} S+M_{N} \bar{N} N+\tilde{\lambda} X \Phi \Phi+ \\
& +S\left(\lambda_{1} R_{d} H_{u}+\lambda \Phi \bar{\Phi}\right)+\lambda_{2} S^{2} N+\tilde{\lambda}_{1} H_{u} L_{a} N
\end{aligned}
$$

where $M_{\Phi} \sim M_{T}$ is the messenger mass scale and $M_{S} \sim M_{N} \sim \sqrt{F}$. The $R$-charge assignment is again the same as the one shown in table 4.3. The superpotential eq.(4.54) will not generate an operator of the form (4.51) since it has a $U(1)$ symmetry under which $\Phi$ and $\bar{\Phi}$ have charge \pm 1 while $X$ as charge -2 . One can also easily show by examining the various spurious $U(1)$ of the superpotential that the $B_{\mu}$ term can only arise at two loops.

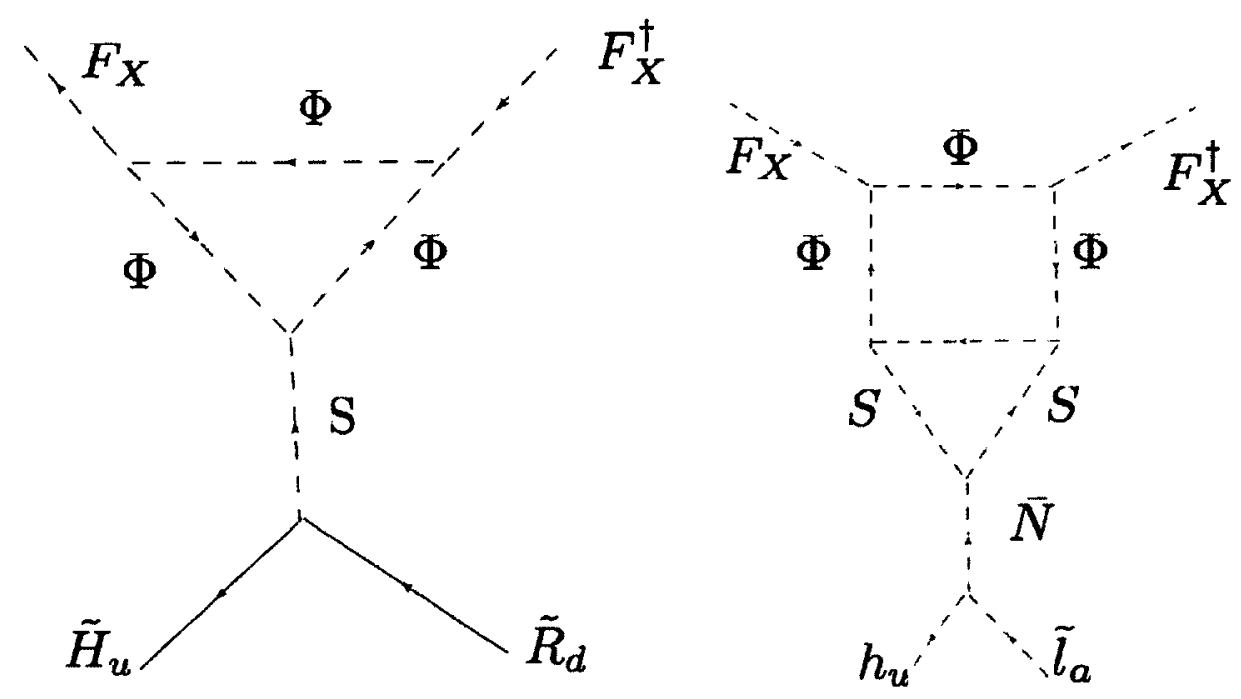

Figure 4.4: Contribution to the $\mu$ and $B_{\mu}$ terms generated by (4.54).

The $\mu$ term on the other hand can be generated from the one loop diagram in 
fig.4.4, and it is given parametrically by:

$$
\mu \sim \frac{\lambda \lambda_{1} \tilde{\lambda}^{2}}{16 \pi^{2}} \frac{F^{2}}{M_{T} M_{S}^{2}} \sim \frac{1}{16 \pi^{2}} \frac{F}{M_{T}},
$$

withs $M_{S} \sim \sqrt{F}$. The $B_{\mu}$ term can instead be generated by the two loops diagram in figure 4.4 , :

$$
B_{\mu} \sim \frac{\tilde{\lambda}_{1} \lambda^{2} \lambda_{2}}{\left(16 \pi^{2}\right)^{2}} \frac{F^{2}}{M_{T}^{2}} \sim \mu^{2} .
$$

Summarizing, this mechanism allows us to generate the $\mu$ term at one loop, and the $B_{\mu}$ together with the scalar masses to be generated at two loops. In order to avoid fine tuning problems we have to introduce a third scale $M_{S}^{2} \sim F$, and several link fields. However, we will see in the section below that these link fields are important also to generate the Yukawa couplings.

To avoid the introduction of the extra link fields, we would need to consider a model $m_{H_{u}}^{2} \gg \mu^{2}, B_{\mu}$, which in an otherwise completely natural model would require some fine-tuning to achieve the correct pattern of electroweak symmetry breaking. However, since, as we have mentioned previously, we already seem to require finetuning to evade the LEP Higgs bound, this hierarchy might in fact not introduce an extra source of fine-tuning (see [95] for a related idea).

\subsubsection{Yukawa coupling for lepton $a$}

As we have already explained, the Yukawa coupling for the lepton of flavor $a$ needs to be generated by the SUSY breaking sector. In models with an $F$-term SUSY breaking spurion, it can be generated by the following operator:

$$
\int \frac{d^{4} \theta}{M^{2}} X^{\dagger} H_{u}^{\dagger} L_{a} l_{a}^{c}
$$

where $X$ is the spurion field whose $F$-term breaks supersymmetry. The Yukawa is then:

$$
y_{a}=\frac{F}{M^{2}}
$$


This type of operator was studied in [96], [97], and can provide the dominant contribution to down-type quarks masses. In the model of [97] for example, it is generated through loops of superpartners. However, in the MMRSSM it is not generated through loops of particles present below the messenger scale, and In order to generate it, it is necessary to enlarge again the messenger sector. We can, for example introduce new link superfields $X_{u}$, and $X_{d}$ with the same gauge numbers of $H_{u}$ and $R_{d}$ respectively, but with different $R$-charges: $X_{u}$ has $R$-charge 2 , while $X_{d}$ has $\mathrm{R}$-charge 0 . They couple to visible sector and messenger fields through superpotential couplings of the form:

$$
W_{y_{a}}=M_{X} X_{u} X_{d}+y_{1} X_{d} H_{u} X+y_{2} X_{d} L_{a} e_{a}^{c}
$$

When $X_{d}$ is integrated out at tree level, it yields the operator of equation (4.57). However, it also yields a tree level contribution to the Higgs soft mass squared. This last contribution can be made smaller than the gauge mediated Higgs soft mass by choosing $y_{1}$ to be small. Then, to generated a large enough Yukawa coupling, the SUSY breaking scale must be rather low. For example, to generate the electron Yukawa $(a=e)$, assuming $M_{X}$ to be of the same order as the messengers and setting the gaugino at $\sim 1 \mathrm{TeV}$, the bound is given by:

$$
\Lambda \lesssim 10^{3} \mathrm{TeV}
$$

In this context, generating the $\tau$ Yukawa would require making $M_{X}$ smaller. Another possibility is to generate the Yukawa at one loop by coupling $X_{u, d}$ to $X$ via the $S$ field of equation (4.54):

$$
W_{y_{a}}=M_{X} X_{u} X_{d}+y_{1} X_{d} L_{a} l_{a}^{c}+y_{2} H_{u} X_{d} \bar{S}+y_{3} X_{u} X_{d} S
$$

where $S$ and $\bar{S}$ are the link fields of eq.(4.54), and we assume $M_{X} \sim M_{S} \sim \sqrt{F}$. Then, the effective following operator receives contribution at one loop:

$$
c \int \frac{d^{4} \theta}{M_{S}^{2} M_{T}^{2}} D^{2}\left(X^{\dagger} X\right) H_{u}^{\dagger} L_{a} l_{a}^{c}
$$


with

$$
c \sim \frac{\lambda \tilde{\lambda}^{2} y_{1} y_{2} y_{3}}{16 \pi^{2}}
$$

but there is no contribution to the Higgs soft mass at the same order. This, taking the gaugino at $1 \mathrm{TeV}$, will give a yukawa coupling of the order of:

$$
y_{a} \sim 10^{2}\left(\frac{1 \mathrm{TeV}}{\Lambda}\right)^{2}
$$

In this way, a Yukawa coupling for the $\tau$ can be accommodated, but requires a low SUSY breaking scale.

\subsection{Phenomelogy}

\subsubsection{MMRSSM at the LHC}

The Dirac nature of the gauginos is one of the most distinctive phenomenological aspects of models with a continuous $R$-symmetry. It could provide a way to distinguish these types of models from the standard SUSY scenario where the gauginos are Majorana fermions. The phenomenology of Dirac gauginos versus Majorana gauginos has been examined in [98]. In addition, the phenomenology of the MRSSM Higgs sector has been recently discussed in [99], and it has been noted that the inert doublet/doublets ${ }^{3}$ with $R$-charge 2 can provide interesting signatures.

The MMRSSM has additional distinguishing features because of the identification of the $U(1)_{R}$ with a lepton number. Because the model does not respect the standard R-parity, the lightest superpartner (LSP) is unstable, as in R-parity breaking models. Since most superpartners are charged under the lepton number $a$, their decay chain will typically produce many leptons. Moreover, in the MMRSSM the LSP is always

\footnotetext{
${ }^{3}$ In our model and in the SOHDM there is just one inert doublet, $H_{d}$, while in the MRSSM a couple $R_{u}$, and $R_{d}$.
} 
the gravitino. As a result, to study the typical decay chain we should look at the next lightest SUSY particle (NLSP). In pure $D$-term SUSY breaking scenarios, the right handed sleptons are typically the lightest particles after the gravitino. Therefore the right handed stau $\tilde{\tau}_{R}$ is the NLSP. When $a=e$ or $a=\mu$, then there are two body decays for $\tilde{\tau}_{R}$ :

$$
\begin{aligned}
& \tilde{\tau}^{ \pm} \rightarrow \nu_{\tau} a^{ \pm}, \\
& \tilde{\tau}^{ \pm} \rightarrow \nu_{a} \tau^{ \pm},
\end{aligned}
$$

Typical decay chains will then contain jets, electron (or muon), plenty of tau's (up to 4), and missing energy from the neutrinos. This kind of signature is also present in $R_{P}$ violating models with $\tilde{\tau}$ LSP (see [100]).

If instead the NLSP is the lightest gaugino $\chi_{1}^{0}$, the situation is a little bit different. The possible $\chi_{1}^{0}$ decay modes are:

$$
\begin{aligned}
& \chi_{1}^{0} \rightarrow Z^{0} \nu_{a}, \\
& \chi_{1}^{0} \rightarrow W^{ \pm} l_{a}^{\mp},
\end{aligned}
$$

which are driven by the mixing with the neutrino $a$. Again, the same phenomenology can be seen in the context of a $R_{p}$ parity violating models.

In summary, the MMRSSM phenomenology is similar to the phenomenology of models with $R_{p}$ violation. However, there are still important differences. First, we can exploit the Dirac nature of the gauginos by looking for example at same sign dileptons signatures. Secondly, as it has been discussed in section 3, the MMRSSM can tolerate a larger level $R_{p}$ parity violation than in the standard $R_{p}$ violating models due to the absence of constraints from neutrino physics. Indeed, in the typical $R_{P}$ violating scenario all decay chains end in the LSP or in the NLSP, whose decay modes are driven by the trilinear $R_{p}$ breaking couplings. Instead, in the MMRSSM the trilinear coupling can be significantly larger and this can lead to a distinctive phenomelogy. 
The most promising channels are the decay of the right handed sbottom and left handed stop ${ }^{4}$, which are the following:

$$
\begin{gathered}
\tilde{b}_{R} \rightarrow b \nu_{a} \\
\tilde{t}_{L} \rightarrow t l_{a}
\end{gathered}
$$

These decay modes can have significant branching ratios, and therefore can lead to interesting signatures typical of leptoquark phenomenology.

Therefore, our model possesses a quite distinctive phenomelogy at colliders, which we will explore it in the a following chapter, but in the next section we will first generalize the model to a framework where the $R$ symmetry corresponds to the global lepton number. We will see that this leads to even more interesting phenomenological consequences both for the LHC and for neutrino physics. Furthermore, it makes more sense to have a global lepton number rather than having to distinguish between two standard lepton number and the $R$ symmetric one.

\section{6 $U(1)_{R}$ as global lepton number}

In the present section we will enlarge the $R$ symmetry to the total lepton number following [3]. This is a more realistic scenario not just because more theoretically motivated, but also because it allows to fit neutrino physics in a natural way as we will see in the next chapter.

\subsubsection{The model}

Let us now describe our framework. We generalize the previous model in such a way that the R-symmetry is identified with the global lepton number, $\mathrm{U}(1)_{R}=U(1)_{L}$. In particular, all the R-charges of Lepton doublets and singlets are respectively fixed to

\footnotetext{
${ }^{4}$ Right handed and Left handed sfermions don't mix in $R$ symmetric models.
} 


\begin{tabular}{l|c|l} 
SuperField & $\left(S U(3)_{c}, S U(2)_{L}\right)_{U(1)_{Y}}$ & $U(1)_{R}$ \\
\hline$Q$ & $(3,2)_{\frac{1}{6}}$ & 1 \\
$U_{i}^{c}$ & $(\overline{3}, 1)_{-\frac{2}{3}}$ & 1 \\
$D_{i}^{c}$ & $(\overline{3}, 1)_{\frac{1}{3}}$ & 1 \\
$E^{c}$ & $(1,1)_{1}$ & 2 \\
$L$ & $(1,2)_{-\frac{1}{2}}$ & 0 \\
$H_{u}$ & $(1,2)_{\frac{1}{2}}$ & 0 \\
$R_{d}$ & $(1,2)_{-\frac{1}{2}}$ & 2 \\
$\Phi_{\bar{W}}$ & $(1,3)_{0}$ & 0 \\
$\Phi_{\tilde{B}}$ & $(1,1)_{0}$ & 0 \\
$\Phi_{\bar{g}}$ & $(8,1)_{0}$ & 0
\end{tabular}

Table 4.4: R-charge assignment for the chiral supermultiplets in our model.

0 and 2, see Table 4.4. The $R_{d}$ electroweak doublet with R-charge 2, introduced to have an anomaly free framework, will play the role of an inert doublet (since we do not want the R-symmetry to be spontaneously broken), while the role of the usual down-type Higgs doublet will be played by a combination of sleptons, as we will explain later on

The most general superpotential compatible with the given $\mathrm{R}$-charge assignment is:

$$
\begin{aligned}
W= & \mu H_{u} R_{d}+H_{u} Q Y_{U} U^{c}+\sum_{i j k} \lambda_{i j k} L_{i} L_{j} E_{k}^{c}+ \\
& +\sum_{i j k} \lambda_{i j k}^{\prime} L_{i} Q_{j} D_{k}^{c}+\lambda_{S} H_{u} \Phi_{\tilde{B}} R_{d}+\lambda_{T} H_{u} \Phi_{\tilde{W}} R_{d} .
\end{aligned}
$$

where $\lambda_{i j k}=-\lambda_{j i k}$ from the antisymmetry of $L_{i} L_{j}$.

The R-conserving SUSY breaking soft lagrangian is instead:

$$
\begin{aligned}
\mathcal{L}_{R}= & m_{H_{u}}^{2} h_{u}^{\dagger} h_{u}+\sum_{i j}\left(m_{L i j}^{2} \tilde{\ell}_{i}^{\dagger} \tilde{\ell}_{j}+m_{R i j}^{2} \tilde{e}_{i}^{\dagger} \tilde{e}_{j}\right)-\sum_{i} b_{\mu}^{i} h_{u} \tilde{\ell}_{i}+ \\
& +\sum_{i j}\left(m_{q i j}^{2} \tilde{q}_{i}^{\dagger} \tilde{q}_{j}+m_{d i j}^{2} \tilde{d}_{i}^{\dagger} \tilde{d}_{j}+m_{u i j}^{2} \tilde{u}_{i}^{\dagger} \tilde{u}_{j}\right)+M_{B} \tilde{B} \tilde{\psi}_{\tilde{B}}+M_{\tilde{W}} \operatorname{tr}\left(\tilde{W}_{\tilde{\psi}} \tilde{\psi}_{\tilde{W}}\right)
\end{aligned}
$$


The R-symmetry cannot be an exact symmetry, since it is broken at least by the gravitino mass necessary to cancel the cosmological constant. To write down the $R$ soft SUSY breaking lagrangian, we need an ansatz on how the R-breaking is communicated to the visible sector. A minimal scenario is to assume that gravity conserves the R-symmetry [72], so that R-breaking effects are communicated to the visible sector only through Anomaly Mediation; however, we can also imagine that gravity effects can break the R-symmetry through Planck suppressed higher dimensional operators.

In the first case, which we will call Anomaly Mediation R-Breaking (AMRB) scenario, the soft R-breaking lagrangian is given by a generalization of eq.(4.41), that is:

$$
\mathcal{L}_{\not \mathscr{R}}^{\mathrm{AMRB}}=\mathcal{L}_{\text {Majorana }}+\mathcal{L}_{\mathrm{A}}+B_{\mu} h_{u} r_{d}
$$

where

$$
\begin{aligned}
\mathcal{L}_{\text {Majorana }} & =m_{B} \tilde{B} \tilde{B}+m_{\tilde{W}} \operatorname{tr}(\tilde{W} \tilde{W})+m_{g} \operatorname{tr}(\tilde{g} \tilde{g}) \\
\mathcal{L}_{A} & =A_{i j k}^{\lambda} \tilde{\ell}_{i} \tilde{\ell}_{j} \tilde{e}_{k}^{c}+A_{i j k}^{D} \tilde{\ell}_{i} \tilde{q}_{j} \tilde{d}_{k}^{c}+h_{u} \tilde{q} A^{U} \tilde{u}^{c} .
\end{aligned}
$$

The first term contains gaugino Majorana masses of order $m \simeq \frac{m_{3 / 2}}{16 \pi^{2}}{ }^{5}$, while the second one contains trilinear scalar interactions proportional to the supersymmetric Yukawa couplings.

Turning to the case in which gravitational effects at the Planck scale break the R-symmetry (which we will call Planck Mediated R-Breaking (PMRB) scenario), the R-breaking structure is much richer than in the previous case, since now all the operators suppressed by some power of the Planck scale can contribute. The Rconserving superpotential and soft SUSY breaking lagrangian, Eqs.(4.71), (4.72), are

\footnotetext{
${ }^{5}$ See [107] for the exact expressions of gaugino Majorana masses and A-terms in Anomaly Mediation.
} 
corrected by the following $R$-contributions:

$$
\begin{aligned}
W_{R}^{\mathrm{PMRB}} & =\sum_{i} \mu_{i} H_{u} L_{i}+\frac{1}{2} m_{T} \operatorname{tr}\left(\Phi_{\bar{W}} \Phi_{\tilde{W}}\right)+\frac{1}{2} m_{S} \Phi_{\bar{B}} \Phi_{\tilde{B}} \\
\mathcal{L}_{\mathscr{R}}^{\mathrm{PMRB}} & =\mathcal{L}_{\text {Majorana }}+\mathcal{L}_{\mathrm{A}}+B_{\mu} h_{u} r_{d} .
\end{aligned}
$$

The $\not R$ soft SUSY breaking contribution has the same structure as in Eq. (4.74), but now we simply expect all the terms generated to be of order of the gravitino mass $m_{3 / 2}$ and the A-terms not to be aligned to the supersymmetric Yukawa couplings. Let us notice the appearance of $\mu$-terms and Majorana masses for the Adjoint Fermions, also of order $m_{3 / 2}$. As we will see, they will play an essential role in neutrino physics.

Let us now study how electroweak symmetry breaking works in this framework and how fermions get masses. Since all the sleptons have a $b_{\mu}$ term, eq. (4.72), in a general basis all sneutrinos will get a vev. However, we can use the freedom to rotate slepton fields to work in a "single vev basis" where just one sneutrino gets a vev $^{6}$. We will denote with $A, B, C$ the flavor indexes in this basis, with $\tilde{L}_{A}$ referring to the doublet that plays the role of the down-type Higgs. The superpotential can be rewritten as:

$$
W=\mu H_{u} R_{d}+\lambda_{S} H_{u} \Phi_{\tilde{B}} R_{d}+\lambda_{T} H_{u} \Phi_{\tilde{W}} R_{d}+W_{\text {Yukawa }}+W_{\text {trilinear }},
$$

with

$$
\begin{aligned}
& W_{\text {Yukawa }}=y_{B} L_{A} L_{B} E_{B}^{c}+y_{C} L_{A} L_{C} E_{C}^{c}+y_{i}^{D} L_{A} Q_{i} D_{i}^{c}+H_{u} Q Y^{U} U^{c} \\
& W_{\text {trilinear }}=\sum_{i=A, B, C} \lambda_{B C i} L_{B} L_{C} E_{i}^{c}+\sum_{i j}\left(\lambda_{B i j}^{\prime} L_{B} Q_{i} D_{j}^{c}+\lambda_{C i j}^{\prime} L_{C} Q_{i} D_{j}^{c}\right)
\end{aligned}
$$

where $y_{B} \equiv \lambda_{A B B}$ and $y_{C} \equiv \lambda_{A C C}$. In the new basis, the R-conserving soft lagrangian of eq. (4.72) maintains the same form, while the R-breaking ones of Eqs. (4.74),(4.75) now read

$$
\mathcal{L}_{\mathrm{A}}=\mathcal{L}_{\mathrm{LR}}+\mathcal{L}_{\text {trilinear }}
$$

\footnotetext{
${ }^{6}$ This is similar to what happens in RPV SUSY models, [78].
} 
with

$$
\begin{aligned}
\mathcal{L}_{\mathrm{LR}} & =A_{B} \tilde{\ell}_{A} \tilde{\ell}_{B} \tilde{e}_{B}^{c}+A_{C} \tilde{\ell}_{A} \tilde{\ell}_{C} \tilde{e}_{C}^{c}+A_{B C} \tilde{\ell}_{A} \tilde{\ell}_{B} \tilde{e}_{C}^{c}+A_{C B} \tilde{\ell}_{A} \tilde{\ell}_{C} \tilde{e}_{B}^{c} A_{i}^{D} \tilde{\ell}_{A} \tilde{q}_{i} \tilde{d}_{i}^{c}+h_{u} \tilde{q} A^{U} \tilde{u}_{i}^{c}, \\
\mathcal{L}_{\text {trilinear }} & =A_{B C i} \tilde{\ell}_{B} \tilde{\ell}_{C} \tilde{e}_{i}^{c}+A_{B i j}^{\prime} \tilde{\ell}_{B} \tilde{q}_{i} \tilde{d}_{j}^{c}+A_{C i j}^{\prime} \tilde{\ell}_{C} \tilde{q}_{i} \tilde{d}_{j}^{c} .
\end{aligned}
$$

The first term gives slepton and squark left-right mixing ${ }^{7}$, while the second term contains trilinear scalar interactions that do not involve the slepton that takes vev. Let us stress that the gaugino Majorana masses and the scalar left/right mixing will play a crucial role in the generation of neutrino masses.

The analysis of the scalar potential can be done along the line of Ref. [78], although in our case the situation is more involved. Indeed, when the left handed slepton soft squared mass matrix is not flavor universal, a mixing between the sneutrino that takes vev and the other two is in principle possible, so that we expect the physical Higgs to be an admixture of all the three sneutrinos. On the contrary, when the squared mass matrix is flavor universal, the resulting scalar potential is the usual one [2]. We assume here for simplicity that, at leading order, the soft squared mass matrix is flavor universal, deferring to a future work the analysis of the non flavor universal case.

From eq. (4.77) we can notice that the charged lepton of flavor $A$ cannot acquire mass through a SUSY invariant Yukawa term as the operator $\ell_{A} \ell_{A} e_{A}^{c}$ is null due to the $S U(2)$ invariance. Therefore, a mass for the lepton $\ell_{A}$ must be generated by a hard SUSY breaking sector through couplings between messengers and leptonic superfields [2]. However, in the present scenario, this sector will generate hard Yukawa couplings also for the $B$ and $C$ flavors.

If we assume that the main contribution to $\ell_{B, C}$ masses comes from the supersymmetric Yukawa couplings, the additional contribution from the hard sector must somehow be suppressed. This makes $A=e$ the simplest possibility. Indeed, if $A=\tau$, the $\tau$

\footnotetext{
${ }^{7}$ In the AMRB scenario the off diagonal terms $A_{B C}, A_{C B}$ are zero.
} 
lepton mass must be generated by the hard sector, while the hard contribution to the other masses must be suppressed (for example requiring the hard Yukawa couplings $y_{i j}$ to satisfy $y_{i j} \ll 10^{-6}$ ). This corresponds to assuming a large hierarchy between the hard Yukawa couplings. The same line of reasoning can be applied in the $A=\mu$ case. If instead $A=e$, a hard Yukawa contribution which generates Yukawa couplings of order $y_{e} \simeq \mathcal{O}\left(10^{-6}\right)$ for all the charged leptons does not give a too large contribution to the $\mu$ and $\tau$ masses, while providing the correct order of magnitude for an electron mass. Since in this case there is no need to introduce any large hierarchy in the new sector, it appears a more natural choice. A possible example of hard Yukawa sector is given in the previous section; however, let us stress that, since we will leave this largely undetermined this sector, in what follows we will analyze also the cases in which $A \neq e$.

As a last comment, let us stress that the interaction terms of $W_{\text {trilinear }}$ (which are not present in the model discussed in the previous section) closely resemble the trilinear interaction terms that appear in RPV theories [78]. However, in our case all

the off diagonal terms involving the flavor $A, \lambda_{A i j}^{\left({ }^{\prime}\right)}$, are zero in the single vev basis, so that the number of parameters is reduced. Moreover, coupling of the type $\lambda_{A i i}^{\left({ }^{\prime}\right)}$ now play the role of Yukawa couplings and are not free parameters. We conclude that our scenario is a variation of RPV models (with less parameters), although as we will see a larger amount of $\mathrm{R}$-parity violation in the neutrino sector than in the standard case will be allowed.

\subsubsection{Electroweak precision measurements and flavor con- straints}

Let us now discuss in turn the experimental constraints coming from Electroweak Precision Measurements (EWPM) and from flavor physics. 
Charged leptons and neutrinos can mix with the "new" spin 1/2 leptons (Dirac gauginos and higgsinos). A priori, the neutralino mass matrix is a $9 \times 9$ squared matrix, while the chargino mass matrix is a $12 \times 12$ square matrix. However, in the single vev basis, the leptons of flavors $B$ and $C$ do not mix with any other fermion, so that the effective matrix is the same as in the previous model. The important point to stress for our purpose is that in the R-symmetric limit all neutrinos are massless. Also, the same bounds on the sneutrino vev coming from the bounds on the coupling of the $Z$ boson to charged leptons apply, i.e. for $M_{\tilde{W}} \sim 1 \mathrm{TeV}$ one should have $v_{A} \lesssim 40 \mathrm{GeV}$.

Let us now turn to the bounds on trilinear couplings appearing in $W_{\text {Yukawa }}$ and $W_{\text {trilinear }}$. Since these are RPV couplings, we refer to $[78,102]$ for a detailed description of the origin of the various bounds and to [103] for updates on these bounds. It is interesting to notice that our framework has distinctive differences both with the model of previous section and with the standard RPV SUSY.

On the one hand, Lepton Flavor Violating (LFV) processes are allowed in our framework but not in the previous version of the model, and the same is true also for semileptonic meson decays (such as rare decays of $B$ and $K$ mesons), unless we assume alignment between the matrices $\left(\lambda_{B, C}^{\prime}\right)_{i j}$ and the quark mass matrix.

On the other hand, even though our situation is more similar to the standard RPV SUSY, some bounds have a different interpretation. In particular, bounds that involve a product between two trilinear couplings can now involve one Yukawa coupling. In order to maximise the parameter space for the sneutrino vev we read these bounds as vev dependent constraints on the trilinear couplings appearing in $W_{\text {trilinear }}$, as shown in table 4.5. For example, when $A=e$, the LFV process $\mu \rightarrow e \gamma$ puts a bound $\left|\lambda_{233}^{*} \lambda_{133}\right| \lesssim 2.3 \times 10^{-4}\left(\frac{m_{\bar{\ell}_{L}}}{100 \mathrm{GeV}}\right)^{2}$ (assuming for simplicity degenerate slepton masses). Since in our model $\lambda_{133}=\frac{m_{\tau}}{v_{e}}$ is the $\tau$ Yukawa coupling, the bound can be restated as $\left|\lambda_{233}\right| \lesssim 2.3 \times 10^{-4} \frac{v_{e}}{m_{\tau}}\left(\frac{m_{\tilde{\ell}_{L}}}{100 \mathrm{GeV}}\right)^{2} \simeq 0.002-0.07$ for $m_{\tilde{\ell}_{L}} \simeq 200 \mathrm{GeV}$ and 
$v_{e}=(10-80) \mathrm{GeV}$. At the same time, it is true that, among the constraints that involve only one trilinear coupling and not a product, some will refer to bounds on Yukawa couplings, implying thus a bound on the sneutrino vev.

In table 4.5 the electroweak precision bounds for the trilinear bounds are reported taken from [78], [102] and [103]. 


\begin{tabular}{|c|c|}
\hline$y_{\mu}=\lambda_{122}$ & $0.05 \tilde{\mu}_{R}$ \\
\hline$y_{\tau}=\lambda_{133}$ & $0.07 \tilde{\tau}_{R}$ \\
\hline$y_{d}=\lambda_{111}^{\prime}$ & $0.02 \tilde{d}_{R}$ \\
\hline$y_{s}=\lambda_{122}^{\prime}$ & $0.21 \tilde{s}_{R}$ \\
\hline$y_{b}=\lambda_{133}^{\prime}$ & $0.18 \tilde{t}_{L}$ \\
\hline$\left|\lambda_{231}\right|$ & $2.98 \frac{v_{e}}{1 G e V} 10^{-4} \tilde{l}_{L}^{2}$ \\
\hline$\left|\lambda_{232}\right|$ & $4 \frac{v_{e}}{1 G e V} 10^{-4} \tilde{l}_{L}^{2}$ \\
\hline$\left|\lambda_{233}\right|$ & $1.32 \frac{v_{e}}{1 G e V} 10^{-4} \tilde{\nu}_{L}^{2}$ \\
\hline$\left|\lambda_{211}^{\prime}\right|$ & $\frac{v_{e}}{1 G e V} 2.110^{-7} \tilde{u}_{L}^{2}$ \\
\hline$\left|\lambda_{222}^{\prime}\right|$ & $1.38 \frac{v_{e}}{1 G e V} 10^{-3} \tilde{d}_{R}^{2}$ \\
\hline$\left|\lambda_{233}^{\prime}\right|$ & $2.71 \frac{v_{e}}{1 G e V} 10^{-5} \tilde{b}_{R}^{2}$ \\
\hline$\left|\lambda_{212}^{\prime}\right|$ & $\frac{v_{e}}{1 \mathrm{GeV}} 610^{-6} \tilde{l}_{L}^{2}$ \\
\hline$\left|\lambda_{213}^{\prime}\right|$ & $1.810^{-4} \tilde{l}_{L}^{2}$ \\
\hline$\left|\lambda_{223}^{\prime}\right|$ & $0.52 \tilde{b}_{R}$ \\
\hline$\left|\lambda_{311}^{\prime}\right|$ & $5 \frac{v_{e}}{1 G e V} 10^{-5} \tilde{l}_{L}^{2}$ \\
\hline$\left|\lambda_{322}^{\prime}\right|$ & $2.7 \frac{v_{e}}{1 G e V} 10^{-4} \tilde{l}_{L}^{2}$ \\
\hline$\left|\lambda_{333}^{\prime}\right|$ & $3.8 \frac{v_{e}}{1 G e V} 10^{-3} \tilde{l}_{L}^{2}$ \\
\hline$\left|\lambda_{312}^{\prime}\right|$ & $6.710^{-5} \tilde{l}_{L}^{2}$ \\
\hline$\left|\lambda_{313}^{\prime}\right|$ & $1.810^{-4} \tilde{l}_{L}^{2}$ \\
\hline$\left|\lambda_{332}^{\prime}\right|$ & $5.3 \frac{v_{e}}{1 \mathrm{GeV}} 10^{-4} \tilde{b}_{R}^{2}$ \\
\hline
\end{tabular}

Table 4.5: The single coupling bounds on the down type Yukawa couplings, and on the interactions $\lambda$ and $\lambda^{\prime}$. 


\subsection{Lepton Yukawa coupling from SUSY breaking}

In this section we will discuss the sector which generates the Yukawa coupling for the lepton of flavor $a$ in the generalised framework of having a global $U(1)_{R}$ lepton number. Therefore, we will generalise what we have discussed in section 1.4.2: As we have already explained, the charged lepton of flavor $a$ does not acquire mass in a SUSY invariant way, but through a hard SUSY breaking Yukawa coupling of form $h_{u}^{\dagger} l_{a} e_{a}^{c}$.

The effective operators that generate a non holomorphic Yukawa coupling are:

$$
\begin{gathered}
c \int \frac{d^{4} \theta}{M^{2}} X^{\dagger} H_{u}^{\dagger} L_{i} E_{j}^{c}, \\
c \int \frac{d^{4} \theta}{M^{4}} D^{2}\left(X^{\dagger} X\right) H_{u}^{\dagger} L_{i} E_{j}^{c},
\end{gathered}
$$

where $i, j=a, b, c$. These operators do not receive any contribution from gauge mediation (also the SUGRA contribution is too small even for the $a=e$ ). Therefore, this coupling needs to be generated by a new SUSY breaking mediation sector that we will call Yukawa sector. This sector inevitably will generate soft scalar masses for the sfermions that could be dangerous for the flavor constraints. We should then add to the SUSY breaking Lagrangian of the model the following hard terms:

$$
\mathcal{L}_{\text {hard }}=\sum_{i j} y_{l i j} h_{u}^{\dagger} l_{i} e_{j}^{c}
$$

and the soft masses:

$$
\mathcal{L}_{Y s o f t}=\sum_{i j}\left(m_{L i j}^{2} l_{i}^{\dagger} l_{j}+m_{R i j}^{2} e_{i}^{\dagger} e_{j}\right)+m_{H_{u}}^{2} h_{u}^{\dagger} h_{u}
$$

There are several constraints these SUSY breaking terms need to fulfil. Let us first focus on the hard Yukawa couplings eq.(4.82). We know that two of three flavors $b, c$ acquire mass through the Yukawa couplings in the superpotential, and therefore the corresponding hard Yukawa couplings should be suppressed compared to the 
SUSY invariant one. This makes $a=e$ the simplest possibility. Indeed when for example $a=\tau$, the hard Yukawa Lagrangian has to contain $y_{\tau}$ of the correct order of magnitude, while all the other couplings need to be suppressed ( for example asking $\left.y_{i j}<<<10^{-6}\right)$. Therefore, the hierarchy between the Yukawa couplings we would have to assume is larger than the one in the SM. If we take $a=e$ instead the Yukawa couplings generated for the muon and tau will have to be roughly at same order of $y_{e}$ in order to avoid the problems mentioned above, so $y_{\tau} \sim y_{\mu} \sim y_{e} \sim y_{i j} \sim 10^{-6}$ with $i, j=e, \mu, \tau$. It is interesting to notice that this implies that the flavor violation introduced in this sector will not be compatible with a MFV hypotesis (neither the RPV version of MFV [104]). We can then assume a different flavor structure or also assume that the Yukawa sector is flavor blind, $y_{\tau} \sim y_{\mu} \sim y_{e} \sim 10^{-6}$. We could also choose to diagonalise the charged lepton mass matrix including all together the hard and the soft Yukawa couplings without imposing such hierarchies. This would open up the possibility $a=\mu$ or $a=\tau$, but it will make the flavor structure of the model rather complicate as the superfield $L_{a}, L_{b}$ and $L_{c}$ will not correspond to the physical leptons, and this would make troublesome for example the determination of the bounds on the RPV couplings.

Now we can turn to the soft masses generated by the Yukawa sector. Naturalness imposes that $m_{H_{u}}^{2}$ and $m_{L_{a}}^{2}$ should be generated at the electroweak scale, while the bounds on flavor violation require the mixing to be suppressed ( at least in the right handed sector $m_{R i j}<<m_{R i i}$, see [71]). The simplest ways out to the flavor problem is to assume the Yukawa sector is flavor blind or it generates soft masses for the sleptons well below the EW scale. As we have already discussed we cannot assume MFV as the hard Yukawa couplings need to obey to a different hierarchy. 


\subsubsection{An example of Yukawa sector}

In this section we want to build a Yukawa sector that fulfil the constraints from naturalness and flavor physics we discussed in the previous section.

A straightforward generalization of eq.(4.54) can be used to build Yukawa coupling in this case:

$$
\begin{gathered}
W_{y_{i}} \quad=M_{S} X_{u} X_{d}+\sum_{i j} y_{l i j} X_{d} L_{i} E_{j}^{c}+y_{2} H_{u} X_{d} \bar{S} \\
+M_{\Phi} \bar{\Phi} \Phi+M_{S} \bar{S} S+\tilde{\lambda} X \Phi \Phi+\lambda \Phi \bar{\Phi} S+\tilde{\lambda}^{\prime} \bar{\Phi} S^{2}
\end{gathered}
$$

There are many more terms allowed by the $R$ symmetry such as couplings between $S$ and the spurion or Yukawa terms such as $X_{u} X_{d} S$. For different reasons we do not want to consider their contributions and therefore we can simply not include them thank to the non renormalizable theorem. This is enough as we are not interested in exploring the UV physics of our model in detail, we just want to extract from the UV the relevant information for the $\mathrm{TeV}$ scale.

Then, the effective operator

$$
c \int \frac{d^{4} \theta}{M_{S}^{2} M^{2}} D^{2}\left(X^{\dagger} X\right) H_{u}^{\dagger} L_{i} E_{j}^{c}
$$

receives contribution at one loop, and the Yukawa couplings are then:

$$
y_{i j} \sim \frac{\lambda \tilde{\lambda}^{2} y_{l i j} y_{2}}{16 \pi^{2}} \frac{F^{2}}{M_{S}^{2} M^{2}} .
$$

The superpotential in eq.(4.85) generate a mass term $m_{H_{u}}^{2}$ at two loops, and at three loops for the sleptons (provided we set to zero the coupling $X_{u} X_{d} S$ ). The contribution of this sector to both the right handed and the left handed sleptons masses is subleading, and therefore we do not need to make any assumptions to the couplings $y_{l i j}$ to avoid possible flavor problems. The leading contribution then comes from gauge mediation. Therefore, for the particular UV completion we just considered 
the slepton mass matrix is flavor universal at leading order.

The model we built contains several ad hoc assumption ( for example: why the couplings $X_{u} X_{d} S$ is absent?), but our purpose was to show that it is possible to disentangle the one loop generation of a Yukawa couplings from the generation of soft masses. As we have explained MFV is not a viable hypotesis in this framework, and therefore other possibilities to have flavor violation in the scalar sector without having FCNC would require looking into flavor model building in more detail. 


\section{Chapter 5}

\section{Fitting neutrino physics with $U(1)_{R}$ lepton number}

In our model the R-symmetry is identified with the global lepton number, so that $U(1)_{R}$ breaking corresponds to lepton number breaking.

The problem of neutrino masses in models with an R-symmetry have been studied both for Majorana [105] and Dirac [106] neutrinos. Both scenarios require to enlarge the particle content of the model by introducing right handed neutrinos. In the standard R-symmetric scenario $[70,75]$, there is no natural connection between the R-breaking and Majorana neutrino masses, since these are allowed by R-symmetry (all lepton superfields have R-charge 1). A priori, however, R-symmetry does not forbid Dirac masses either, since their presence depend on the R-charge assignment of right-handed neutrinos.

On the contrary, in our scenario there is a clear connection between Majorana neutrino masses and R-breaking effects, since such Majorana masses are clearly incompatible with the $U(1)_{R}$ symmetry. In this way, in principle we don't need to introduce any additional particle (i.e. right-handed neutrinos) in order to generate non zero masses. While, as we will see, this will be true for AMRB, in the case of PMRB additional structure will be necessary in order to reproduce neutrino masses and mixing, making 


\begin{tabular}{|c|c|c|}
\hline Quantity & ref. [108] & ref. [109] [110] \\
\hline$\Delta m_{\text {sun }}^{2}\left(10^{-5} \mathrm{eV}^{2}\right)$ & $7.58_{-0.26}^{+0.22}$ & $7.59_{-0.18}^{+0.20}$ \\
\hline$\Delta m_{\text {atm }}^{2}\left(10^{-3} \mathrm{eV}^{2}\right)$ & $2.35_{-0.09}^{+0.12}$ & $\begin{array}{c}2.50_{-0.16}^{+0.09} \\
-\left(2.40_{-0.09}^{+0.08}\right)\end{array}$ \\
\hline $\sin ^{2} \theta_{12}$ & $0.312_{-0.016}^{+0.017}$ & $0.312_{-0.015}^{+0.017}$ \\
\hline $\sin ^{2} \theta_{23}$ & $0.42_{-0.03}^{+0.08}$ & $0.52_{-0.06}^{+0.06}$ \\
\hline $\sin ^{2} \theta_{13}$ & $0.025 \pm 0.007$ & $0.013_{-0.005}^{+0.007}$ \\
& & $0.016_{-0.006}^{+0.008}$ \\
\hline
\end{tabular}

Table 5.1: Fits to neutrino oscillation data. Where two different values are present for one parameter, upper and lower row refer respectively to Normal and Inverted Hierarchy.

this scenario less compelling.

Let us stress again that in our scenario the scale at which Lepton Number is broken is deeply connected with the scale of supersymmetry breaking through the gravitino mass, while in general the Majorana neutrino masses generated through the Weinberg operator call for a very large scale, which may or may not be connected to the scale of supersymmetry breaking.

\subsubsection{Neutrino masses and mixings}

Before analyzing the neutrino phenomenology in our framework, let us briefly summarize some features of a general neutrino mass matrix.

As it is well known, the neutrino mass matrix is largely undetermined, since we lack of information on the absolute neutrino mass scale and on the hierarchy between the mass eigenstates. For three active neutrinos, the present data are summarized in Table 5.1.

At the same time, CMB data point towards $\sum_{i} m_{\nu, i} \lesssim 0.6 \mathrm{eV}$ (see e.g. [111]), from 
which one can infer a loose upper bound $m_{\text {lightest }} \lesssim 0.1 \mathrm{eV}$ for both hierarchies. Using data in the expression of the neutrino mass matrix in terms of masses and mixing, we expect the following general form for the mass matrix (in the $\left(\nu_{e}, \nu_{\mu}, \nu_{\tau}\right)$ basis) [112],

(*) Normal Hierarchy:

$$
M_{\nu} \simeq m_{\nu}^{\text {small }}\left(\begin{array}{ccc}
\mathcal{O}(\varepsilon) & \mathcal{O}(\varepsilon) & \mathcal{O}\left(\varepsilon^{2}\right) \\
\mathcal{O}(\varepsilon) & \mathcal{O}(1) & \mathcal{O}(1) \\
\mathcal{O}(\varepsilon) & \mathcal{O}(1) & \mathcal{O}(1)
\end{array}\right), M_{\nu} \simeq m_{\nu}^{\text {large }}\left(\begin{array}{ccc}
\mathcal{O}(1) & \mathcal{O}(\epsilon) & \mathcal{O}(\epsilon) \\
\mathcal{O}(\epsilon) & \mathcal{O}(1) & \mathcal{O}(\sqrt{\epsilon}) \\
\mathcal{O}(\epsilon) & \mathcal{O}(\sqrt{\epsilon}) & \mathcal{O}(1)
\end{array}\right)
$$

(*) Inverted Hierarchy:

$$
M_{\nu} \simeq m_{\nu}^{\text {small }}\left(\begin{array}{ccc}
\mathcal{O}(1) & \mathcal{O}(\varepsilon) & \mathcal{O}(\varepsilon) \\
\mathcal{O}(\varepsilon) & \mathcal{O}(1) & \mathcal{O}(1) \\
\mathcal{O}(\varepsilon) & \mathcal{O}(1) & \mathcal{O}(1)
\end{array}\right), \quad M_{\nu} \simeq m_{\nu}^{\text {large }}\left(\begin{array}{ccc}
\mathcal{O}(1) & \mathcal{O}(\epsilon) & \mathcal{O}(\epsilon) \\
\mathcal{O}(\epsilon) & \mathcal{O}(1) & \mathcal{O}(\sqrt{\epsilon}) \\
\mathcal{O}(\epsilon) & \mathcal{O}(\sqrt{\epsilon}) & \mathcal{O}(1)
\end{array}\right)
$$

The matrices on the left and on the right refer respectively to a small (of order $\left.\mathcal{O}\left(10^{-5} \mathrm{eV}\right)\right)$ and large (of order $\left.\mathcal{O}\left(10^{-1} \mathrm{eV}\right)\right)$ lightest neutrino mass. The exact value of the coefficients depends on the chosen values of neutrino masses and mixing angles (we discard here the dependence on Dirac and Majorana phases); however, typical order of magnitudes for the elements are

$$
m_{\nu}^{\text {small }} \simeq \mathcal{O}\left(10^{-2} \mathrm{eV}\right), \quad m_{\nu}^{\text {large }} \simeq \mathcal{O}\left(10^{-1} \mathrm{eV}\right), \quad \varepsilon \simeq \mathcal{O}\left(10^{-1} \mathrm{eV}\right), \quad \epsilon \simeq \mathcal{O}\left(10^{-3} \mathrm{eV}\right)
$$

In what follows, taking the approach of [112], we will focus on specific forms for the neutrino mass matrix that we consider representative of the different phenomenological scenarios. In particular, we will focus on the two following matrices, representative 
respectively of the Normal and Inverted Hierarchy cases for small lightest neutrino mass:

$$
m_{\nu}^{\mathrm{NH}} \simeq 10^{-2}\left(\begin{array}{ccc}
0.39 & 0.80 & 0.017 \\
0.80 & 2.99 & 2.08 \\
0.017 & 2.08 & 2.49
\end{array}\right) \mathrm{eV} ; m_{\nu}^{\mathrm{IH}} \simeq 10^{-2}\left(\begin{array}{rrr}
4.7 & -0.54 & -0.52 \\
-0.54 & 2.19 & -2.36 \\
-0.52 & -2.36 & 2.8
\end{array}\right) \mathrm{eV}
$$

We do not show here the corresponding matrices for the large lightest neutrino mass scenario because, as we will explain later on, they can be reproduced only in a very small region of parameter space. To construct the previous matrices, we fixed the lightest neutrino mass to $2 \times 10^{-5} \mathrm{eV}$, while the other parameters are fixed as follows: $\Delta m_{12}^{2} \simeq 7.6 \times 10^{-5} \mathrm{eV}^{2}, \Delta m_{13}^{2} \simeq 2: 4 \times 10^{-3} \mathrm{eV}^{2}, \sin ^{2} \theta_{12} \simeq 0.3, \sin ^{2} \theta_{23} \simeq 0.47$,

$\sin ^{2} \theta_{13} \simeq 0.024$, i.e. we take $\theta_{13} \simeq 9^{\circ}$ as recently observed by the Daya Bay collaboration [113] and by RENO collaboration [114]. For simplicity, we have also assumed a vanishing $\mathrm{CP}$ violating phase.

\subsubsection{Neutrino physics in AMRB}

Inspecting Eq. (4.73), it is clear that the gaugino Majorana masses contribute to the neutralino-neutrino mass matrix. This resembles what happens in RPV theories with bilinear terms [78], where one neutrino gets a non zero mass already at tree level through its mixing with gauginos.

On the contrary, in this scenario all neutrinos remain massless at tree level. This is a striking difference with respect to the RPV case, and can be understood considering the approximate eigenstates of the neutrino mass matrix (calculated e.g. using the 

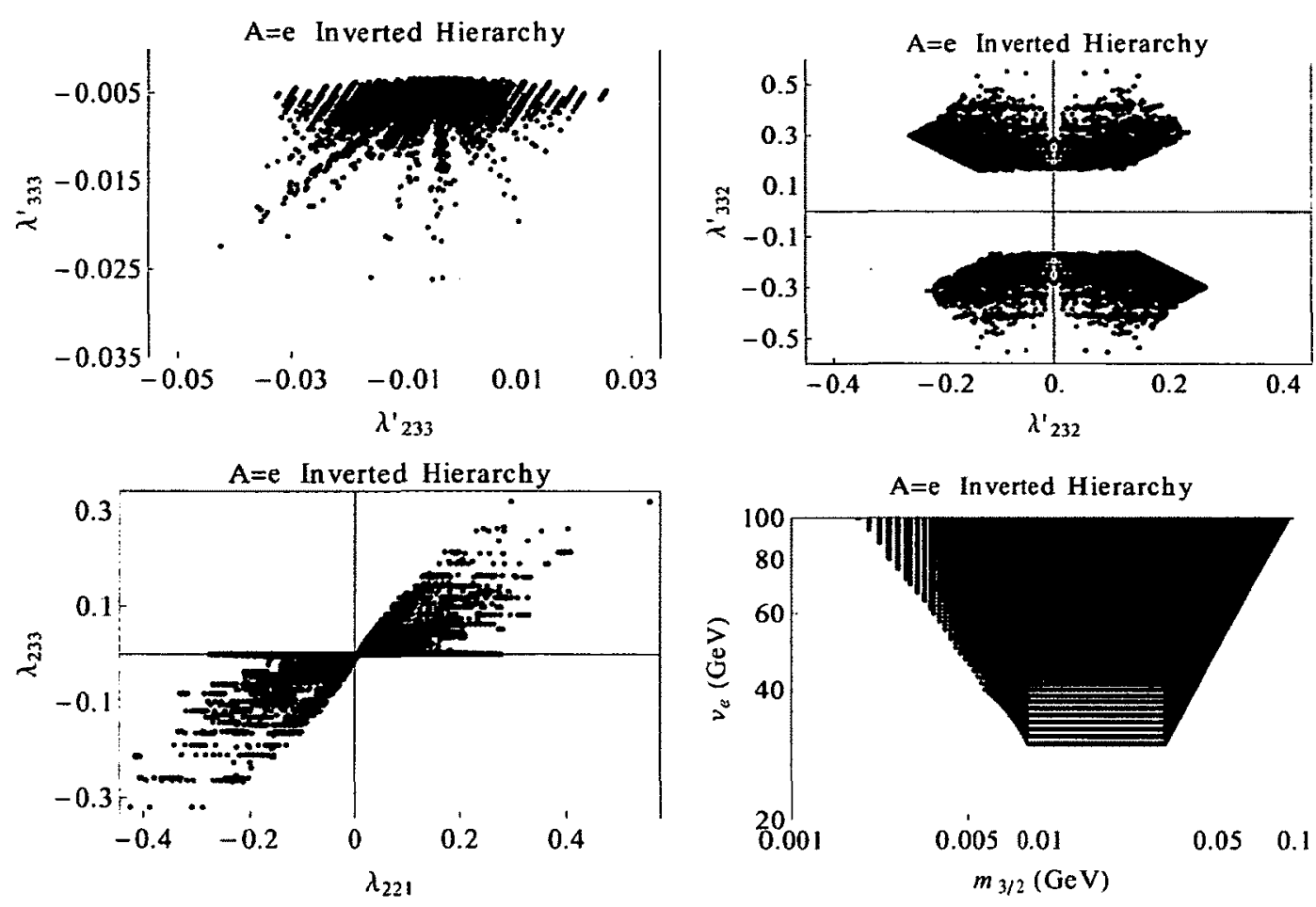

Figure 5.1: Allowed region (colored) in parameter space for the flavor assignment $A=e, B=\mu$ and $C=\tau$ in the case of Inverted Hierarchy.

usual see-saw formula):

$$
\left(\begin{array}{c}
\nu_{A}^{\prime} \\
\nu_{B}^{\prime} \\
\nu_{B}^{\prime}
\end{array}\right) \simeq\left(\begin{array}{c}
\nu_{A}+\frac{g v_{A} v_{u} \lambda_{T}}{\sqrt{2} \mu M_{\tilde{W}}} \tilde{h}_{u}+\frac{g^{\prime} v_{A}}{\sqrt{2} M_{B}} \tilde{\psi}_{B}-\frac{g v_{A}}{\sqrt{2} M_{\tilde{W}}} \tilde{\psi}_{W} \\
\nu_{B} \\
\nu_{C}
\end{array}\right)
$$

The $B$ and $C$ flavors are by themselves approximate eigenstates and cannot get mass through a mixing with gauginos. At the same time, the flavor $A$ mixes only with Higgsinos and adjoint fermions, so that the absence of mixing with gauginos and of Majorana masses for the adjoint fermions prevents $\nu_{A}$ from getting a tree level mass.

It is now clear that, in the AMRB scenario, the only possibility for neutrinos to acquire a mass is through loop effects. In the $\left(\nu_{A}, \nu_{B}, \nu_{C}\right)$ basis, the main contributions at 1-loop are given by [78]: 
- Loops with two supersymmetric trilinear couplings and one mass insertion in the scalar propagator due to Anomaly Mediation.

Since this term is proportional to the mass of the fermion circulating in the loop, the dominant contributions are given by bottom quark, strange quark and tau lepton ${ }^{1}$.

They are given by:

$$
\begin{aligned}
m_{\nu}^{q}= & \frac{6}{\left(16 \pi^{2}\right)^{2}}\left(\frac{m_{3 / 2} v_{A}}{m_{\tilde{b}}^{2}}\right) \hat{\beta}_{b}\left[m_{b}\left(\begin{array}{ccc}
\lambda_{A 33}^{\prime 2} & \lambda_{A 33}^{\prime} \lambda_{B 33}^{\prime} & \lambda_{A 133}^{\prime} \lambda_{B 33}^{\prime} \\
\lambda_{133}^{\prime} \lambda_{B 33}^{\prime} & \lambda_{B 33}^{\prime 2} & \lambda_{C 33}^{\prime} \lambda_{B 33}^{\prime} \\
\lambda_{A 33}^{\prime} \lambda_{C 33}^{\prime} & \lambda_{C 33}^{\prime} \lambda_{B 33}^{\prime} & \lambda_{C 33}^{\prime 2}
\end{array}\right)+\right. \\
& \left.m_{s}\left(\begin{array}{ccc}
0 & 0 & 0 \\
0 & \lambda_{B 32}^{\prime 2} & \lambda_{B 32}^{\prime} \lambda_{C 32}^{\prime} \\
0 & \lambda_{B 32}^{\prime} \lambda_{C 32}^{\prime} & \lambda_{C 32}^{\prime 2}
\end{array}\right)\right]
\end{aligned}
$$

where $\lambda_{A i i}^{\prime}=\left(m_{d}\right)_{i} / v_{A}$ is the $i^{t h}$ down-quark Yukawa coupling, $m_{\tilde{b}}$ is the common left handed and right handed sbottom mass scale, $\hat{\beta}_{b}$ is the bottom $\beta$ function, and for simplicity we have assumed $\lambda_{B 23}^{\prime}=\lambda_{B 32}^{\prime}, \lambda_{C 23}^{\prime}=\lambda_{C 32}^{\prime}$.

In the lepton sector, the main contribution is given by

$$
m_{\nu}^{\tau}=\frac{2}{\left(16 \pi^{2}\right)^{2}}\left(\frac{m_{\tau} m_{3 / 2} v_{A}}{m_{\bar{\tau}}^{2}}\right) \hat{\beta}_{\tau}\left(\begin{array}{ccc}
\lambda_{A 33}^{2} & \lambda_{A 33} \lambda_{B 33} & 0 \\
\lambda_{A 33} \lambda_{B 33} & \lambda_{B 33}^{2} & -\lambda_{B 33} \lambda_{C 33} \\
0 & -\lambda_{B 33} \lambda_{C 33} & 0
\end{array}\right)
$$

where $\lambda_{A 33}=m_{\tau} / v_{A}$ is the tau Yukawa coupling and $\hat{\beta}_{\tau}$ the tau $\beta$-function.

\footnotetext{
${ }^{1}$ We neglect the muon contribution because, due to color factors, it is subdominant with respect to the strange quark contribution.
} 
- Loops with two gauge couplings and one Majorana mass insertion in the gaugino propagator:

$$
\left(m_{\nu}^{\mathrm{gg}}\right)_{A A}=\frac{g^{4}}{4} \frac{m_{3 / 2}}{\left(16 \pi^{2}\right)^{2}}\left(\frac{v_{A}}{v}\right)^{2} \frac{m_{Z}^{2}}{M_{\bar{W}}^{2}}
$$

where $M_{\tilde{W}}$ is the Dirac Wino mass and we have used the Anomaly Mediation contribution to the Majorana Wino mass: $m_{\tilde{W}}=\frac{g^{2}}{16 \pi^{2}} m_{3 / 2}$.

In the previous equations we neglected the mixing of $\nu_{a}$ with the adjoint gauginos (see Eq. (5.4)): this is consistent in the portion of parameter space we will consider in the following numerical analysis.

Barring special relationship between the parameters involved, the neutrino mass matrix has now three non zero eigenvalues. These depend on free parameters (trilinear RPV couplings and gravitino mass), that can be chosen to fit the experimental data, but also on gauge couplings and masses which are constrained by collider experimental bounds ${ }^{2}$.

As already stressed, our scenario is a particular case of RPV SUSY (in particular the loop contributions are the same in both cases), so that it is interesting to compare the two situations. Usually in RPV scenarios the left/right sparticle mixing and the Majorana gauginos mass are at the EW scale, while in our case they are proportional to the gravitino mass and can be subleading for small supersymmetry breaking scale. This implies that while one usually needs to suppress too large loop contributions to neutrino masses by putting severe upper bounds on the trilinear couplings [78], in our case the upper bound is translated to the gravitino mass (with trilinear couplings usually allowed to saturate the bounds from EWPM and flavor physics, see Sec.

\footnotetext{
${ }^{2}$ In what follows we will always take as reference a "natural" spectrum for the supersymmetric partners, with only the squarks of the third generation below the TeV scale, while all other superparticle masses can be above the $\mathrm{TeV}$ scale. At the moment, the experimental bounds on this kind of spectrum is less severe than those obtained for almost degenerate squarks [60]. Note that Dirac gauginos have an improved naturalness with respect to Majorana gauginos [64], and this allows us to have a natural gluino above the $\mathrm{TeV}$ scale and a heavier Wino. In what follows, we will take the Dirac Wino mass up to $10 \mathrm{TeV}$.
} 


\begin{tabular}{|c|c|c|c|c|c|}
\hline$A=e$ & $\left|\lambda_{133}\right|$ & $v_{A}(\mathrm{GeV})$ & $m_{\tilde{b}}(\mathrm{GeV})$ & $m_{\tilde{\tau}}(\mathrm{GeV})$ & $M_{\tilde{W}}(\mathrm{TeV})$ \\
\hline \multirow[b]{2}{*}{$A=\mu$} & $5 \times 10^{-7}-1.4$ & $20-100$ & $300-1000$ & $200-1000$ & $0.5-10$ \\
\hline & $\left|\lambda_{233}\right|$ & $v_{A}(\mathrm{GeV})$ & $m_{\tilde{b}}(\mathrm{GeV})$ & $m_{\tilde{\tau}}(\mathrm{GeV})$ & $M_{\tilde{W}}(\mathrm{TeV})$ \\
\hline \multirow[b]{2}{*}{$A=\tau(i)$} & $5 \times 10^{-7}-1.4$ & $20-100$ & $300-1000$ & $200-1000$ & $0.5-10$ \\
\hline & & $v_{A}(\mathrm{GeV})$ & $m_{\tilde{b}}(\mathrm{GeV})$ & $m_{\tilde{\tau}}(\mathrm{GeV})$ & \\
\hline \multirow{3}{*}{$A=\tau(i i)$} & & $20-100$ & $300-1000$ & $200-1000$ & \\
\hline & & $v_{A}(\mathrm{GeV})$ & $m_{\tilde{b}}(\mathrm{GeV})$ & $m_{\bar{\tau}}(\mathrm{GeV})$ & $M_{\tilde{W}}(\mathrm{TeV})$ \\
\hline & & $20-100$ & $300-1000$ & $200-1000$ & $0.5-10$ \\
\hline
\end{tabular}

Table 5.2: Range of parameters used in the scan of Secs. 5.0.3-5.0.3.

\subsection{2).}

A loose upper bound on the gravitino mass can be derived from cosmological considerations. Indeed, as already stressed, the absolute neutrino mass scale is bounded from above from CMB measurements, $m_{\nu} \lesssim 0.6 \mathrm{eV}$. This readily translates into an upper bound on the gravitino mass, which can be roughly estimate as follows. Since $m_{A A}$ is the only entry in the neutrino mass mass matrix that does not depend on trilinear couplings, we can use it to roughly set the largest neutrino eigenvalue scale. For typical value of sparticle masses $\left(m_{\tilde{b}, \tilde{\tau}} \lesssim 1 \mathrm{TeV}, M_{\tilde{W}} \lesssim 10 \mathrm{TeV}\right)$ we obtain

$$
m_{3 / 2} \lesssim 0.5 \mathrm{GeV}
$$

We will now study in detail whether, in the AMRB scenario, the phenomenological neutrino mass matrices can be reproduced in the case where the flavor $A$ is the either electron, muon or tau. 


\section{$A=e:$ Electronic Higgs}

In this case we assign $A=e, B=\mu, C=\tau$. We perform our numerical scan for the parameters of Table 5.2, requiring the other variables to reproduce the phenomenological matrices and imposing the constraints of [102]. For simplicity, we have assumed degeneracy between LH and RH sparticles, and a full family degeneracy in the slepton sector. Strictly speaking, this simplification implies that, barring accidental cancellations, a natural common slepton mass cannot be too large, since it enters in the determination of the $\mathrm{Z}$ mass through the minimization of the scalar potential. However, keeping in mind that only the LH slepton mass matrix affects the Higgs sector, and to have an idea of the general behavior of the model, we allow the common slepton mass to assume also larger values.

The main result of this section is that while the Normal Hierarchy case can be reproduced only in a very small region of the parameter space (corresponding to $v_{e} \sim$ $100 \mathrm{GeV}$ and rather large Dirac Wino masses, $M_{\tilde{W}} \gtrsim 5 \mathrm{TeV}$ ), a much larger portion of parameter space is available for the Inverted Hierarchy. This can be understood by looking at the phenomenological matrices of Eq. (5.3): the $m_{e e}$ entry in the Normal Hierarchy case is about one order of magnitude smaller than the one of the Inverted Hierarchy case. For this to happen, one needs large sneutrino vev and large Wino mass. This can be seen by noting that we can parametrize $m_{e e}$ as

$$
m_{e e} \propto\left(\frac{\alpha}{v_{e}}+\beta v_{e}^{2}\right) m_{3 / 2}
$$

where the first term comes from the squark and slepton loops $\left(\alpha \sim 1 / \tilde{m}^{2}\right)$ while the second one is due to the Wino loop $\left(\beta \sim 1 / M_{\bar{W}}^{2}\right)$. A large vev can suppress the first term, while a large Wino mass can suppress the largeness of the second one. The available parameter space for Inverted Hierarchy is shown in Fig. 5.1, where the allowed region is the colored one. We do not show plots in the sparticle and Wino mass planes since these parameters are practically unconstrained. As can be seen, 
in the squark sector the diagonal trilinear couplings $\lambda_{333}^{\prime}, \lambda_{233}^{\prime}$ are rather small, both at most of order $\mathcal{O}\left(10^{-2}\right)$, while the off-diagonal trilinear couplings $\lambda_{332}^{\prime}, \lambda_{232}^{\prime}$ can be large, up to $\mathcal{O}\left(10^{-1}\right)$. In the lepton sector we have again couplings $\lambda_{233}, \lambda_{231}$ at most of order $\mathcal{O}\left(10^{-1}\right)$.

As a result of our scan of the parameter space, we can set a more precise range on the gravitino mass:

$$
1 \mathrm{MeV} \lesssim m_{3 / 2} \lesssim 100 \mathrm{MeV}
$$

Furthermore, we also have an indication on the sneutrino vev: we can fit the neutrino mass matrix in our framework only if the sneutrino vev is somewhat large, $v_{e} \gtrsim$ $30 \mathrm{GeV}$, i.e. $\tan \beta \equiv \frac{v_{u}}{v_{e}} \lesssim 6$. Let us also notice that for larger sneutrino vev, a larger gravitino mass is allowed. This can be understood from Eq. (5.8), from which it is clear that for small sneutrino vevs the term between brackets can be large, so that in general a small gravitino mass is needed to suppress this entry. On the contrary, for larger values of the vev the term between brackets is more suppressed, and a larger gravitino mass is allowed.

A comment on the situation for larger lightest $m_{\text {lightest }}$ is in order. We have explicitly checked the situation for $m_{\text {lightest }} \simeq 0.1 \mathrm{eV}$, finding that the phenomenological neutrino mass matrix can be reproduced only in very a small region of parameter space. However, let us stress that in this case approximately the same region of parameter space can reproduce both Hierarchies, since now the typical form of the mass matrix in the two cases is similar (Eqs. (5.1), (5.2)). 

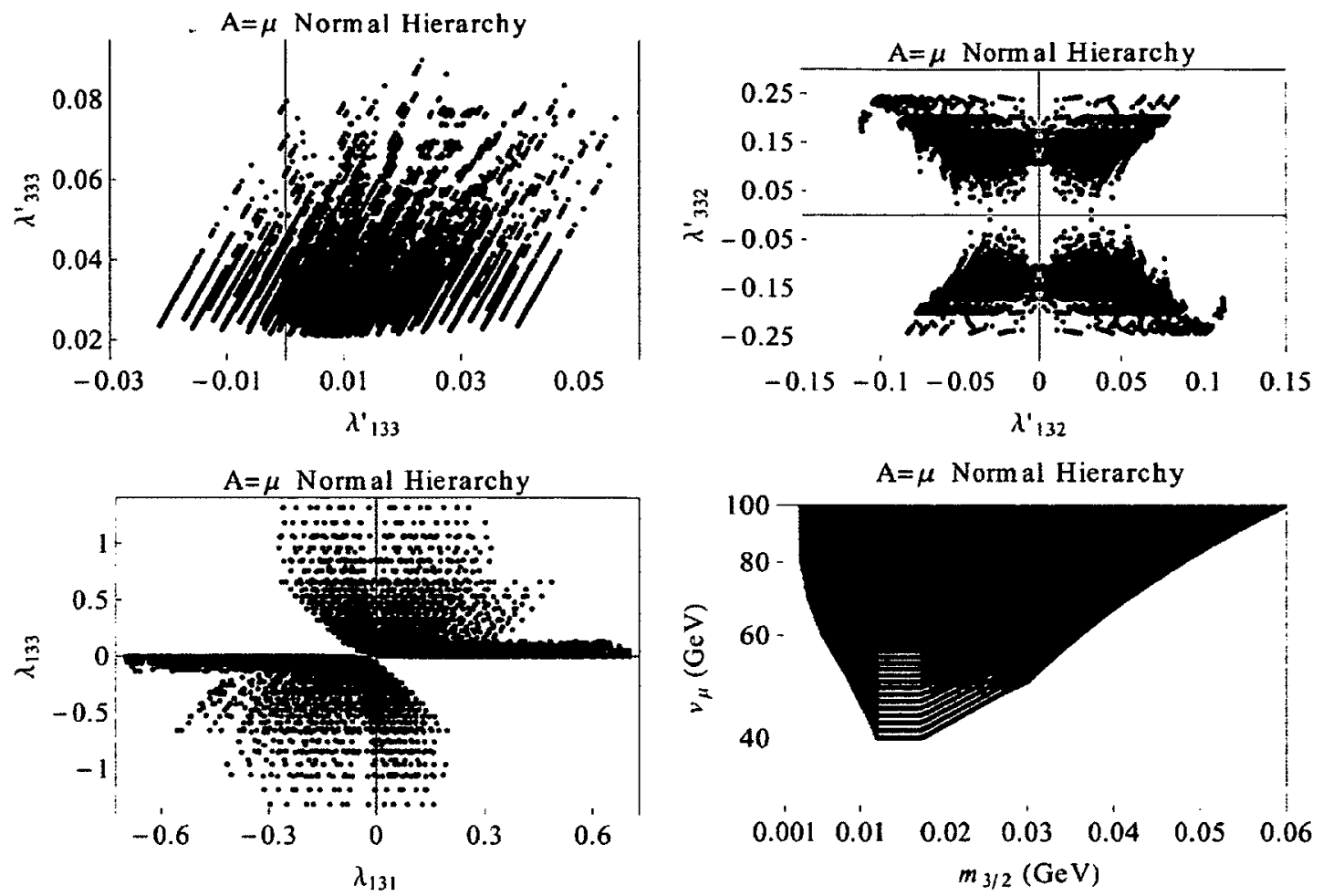

Figure 5.2: Allowed region (colored) in parameter space for the flavor assignment $A=\mu, B=e$ and $C=\tau$ in the case of Normal Hierarchy.

\section{$A=\mu, \tau:$ Muon and Tau Higgs}

As pointed out in Sec. 4.6.1, we consider the case of an Electronic-Higgs $(A=e)$ more motivated from the point of view of the generation of the hard Yukawa couplings. However, for completeness we study also other possibilities. In particular, as we will see, the $A=\mu$ case offers an interesting different phenomenological situation with respect to the $A=e$ case.

Let us start with $A=\mu, B=e$ and $C=\tau$. In this case Eq. (5.8) is valid for $m_{\mu \mu}$, which is similar for the two hierarchies. Thus, in general we expect that, unlike what happens in the $A=e$ case, both Hierarchies should be reproduced. This is indeed what happens, as confirmed by the scan performed for the parameters of Table 5.2 within the same approximations described in the previous section.

The results are shown in Figs. 5.2-5.3. Also in this case we have checked that 



Figure 5.3: Allowed region (colored) in parameter space for the flavor assignment $A=\mu, B=e$ and $C=\tau$ in the case of Inverted Hierarchy.

increasing the lightest neutrino mass diminishes drastically the available parameter space (although also in this case both Hierarchies can be accommodated).

As can be seen from the plots, the range of parameters is roughly the same as the $A=e$ case, although some details can change. An exception is given by the lepton trilinear coupling $\lambda_{133}$, which is now allowed to be also of $\mathcal{O}(1)$. Regarding the muon-sneutrino vev and the gravitino mass, interestingly the situation does not change much with respect to the $A=e$ case: we conclude that the bounds of Eq. (5.9) are rather typical, for small neutrino masses, while they are no longer valid when the lightest neutrino mass is increased.

Let us now comment on the Tau-Higgs case, i.e. $A=\tau$. We have performed our analysis both in the approximation of vanishing (i) and non vanishing (ii) muon mass. In the case (i) there is no contribution from loops involving sleptons, so that 
one can solve for the Dirac Wino mass instead of scanning on it. The results of our scan show that a solution compatible with the phenomenological mass matrices, Eq. (5.3) requires either very large trilinear couplings or very large Wino masses (well above $100 \mathrm{TeV}$ ). While the first possibility is excluded by the bounds coming from EWPM [102], the second one is in principle viable. However, since as we already pointed out we want to stick to a spectrum which is not too unnatural, we consider this possibility at best marginal.

In the case (ii) there is a non vanishing slepton loop contribution, in such a way that the scan on parameter space is quite similar to those of the two previous Sections (with the exception that in this case one of the two trilinear coupling constants involved is the muon-Yukawa coupling, so that there is no need to scan over it). Nevertheless, also in this case compatibility with Eq. (5.3) requires trilinear couplings incompatible with the bounds of [102].

The situation is summarized in Table 5.2; and the conclusion is that the case $A=\tau$ cannot reproduce neither a Normal nor a Inverted Hierarchy spectrum.

\subsubsection{Neutrino physics in PMRB}

Let us now turn to the case where gravitational effects also break the $U(1)_{R}$ symmetry. The main difference with the previous case is that now two non zero neutrino masses are generated at tree level. To understand this, let us consider the mixing among fermions in the neutralino sector. In the $\mathrm{R}$-symmetric limit, the $R=-1$ mass eigenstates are well approximated by:

$$
\begin{aligned}
\nu_{A}^{\prime} & \simeq \nu_{A}-\frac{g v_{A}}{\sqrt{2} M_{W}} \psi_{\tilde{W}}+\frac{g^{\prime} v_{A}}{\sqrt{2} M_{B}} \psi_{\tilde{B}}+\frac{g v_{A} v_{u} \lambda_{T}}{\sqrt{2} \mu M_{\tilde{W}}} \\
\psi_{\tilde{W}^{\prime}} & \simeq \psi_{\tilde{W}}-\frac{g v_{A}}{\sqrt{2} M_{W}} \nu_{A}-\frac{g v_{u}}{\sqrt{2} M_{B}} \tilde{h}_{u} \\
\psi_{\tilde{B}^{\prime}} & \simeq \psi_{\tilde{B}}-\frac{g^{\prime} v_{A}}{\sqrt{2} M_{W}} \nu_{A}-\frac{g v_{u}}{\sqrt{2} M_{B}} \tilde{h}_{u}
\end{aligned}
$$


while $\nu_{B}$ and $\nu_{C}$ do not mix, as we have already noticed. The $R=1$ states are instead:

$$
\tilde{W}^{\prime} \simeq \tilde{W}-\frac{\lambda_{T}}{\sqrt{2} M_{W}} \tilde{h}_{d}, \quad \tilde{B}^{\prime} \simeq \tilde{B}-\frac{\lambda_{S}}{\sqrt{2} M_{B}} \tilde{h}_{d} .
$$

The inclusion of $\mathrm{R}$-breaking effects generates new mixing terms for all neutrinos:

$$
\begin{aligned}
\nu_{A}^{\prime} & \simeq \nu_{A}-\frac{g v_{A}}{\sqrt{2} M_{W}} \psi_{\tilde{W}}+\frac{g^{\prime} v_{A}}{\sqrt{2} M_{B}} \psi_{\tilde{B}}+\frac{g v_{A} v_{u} \lambda_{T}}{\sqrt{2} \mu M_{\tilde{W}}}-\frac{\mu_{A}}{\mu} \tilde{h}_{d}, \\
\nu_{B} & \simeq \nu_{B}-\frac{\mu_{B}}{\mu} \tilde{h}_{d} \\
\nu_{C} & \simeq \nu_{B}-\frac{\mu_{C}}{\mu} \tilde{h}_{d},
\end{aligned}
$$

which in turn produce a mass term for $\nu_{A}$ and mixing terms $m_{A B}, m_{A C}$ :

$$
\begin{aligned}
m_{A A} & =\sqrt{2} \frac{v_{A} v_{u}}{\mu}\left(\frac{g \lambda_{T}}{M_{\tilde{W}}}-\frac{g^{\prime} \lambda_{S}}{M_{B}}\right) \mu_{A}, \\
m_{A B} & =\frac{v_{A} v_{u}}{\sqrt{2} \mu}\left(\frac{g \lambda_{T}}{M_{\tilde{W}}}-\frac{g^{\prime} \lambda_{S}}{M_{B}}\right) \mu_{B} \\
m_{A C} & =\frac{v_{A} v_{u}}{\sqrt{2} \mu}\left(\frac{g \lambda_{T}}{M_{\tilde{W}}}-\frac{g^{\prime} \lambda_{S}}{M_{B}}\right) \mu_{C} .
\end{aligned}
$$

Furthermore, a Majorana mass for the adjoint gauginos is generated, and through it the neutrino $\nu_{A}$ acquire an additional mass term:

$$
m_{A}=\left(\frac{g v_{A}}{M_{W}}\right)^{2} m_{T}+\left(\frac{g^{\prime} v_{A}}{M_{B}}\right)^{2} m_{S} .
$$

This is an example of inverse see-saw mechanism [115], where the role of the right handed Dirac neutrinos is played by the Dirac gauginos. Therefore, the tree level mass matrix in the PMRB scenario is:

$$
\begin{aligned}
m_{A A} & =\sqrt{2} \frac{v_{A} v_{u}}{\mu}\left(\frac{g \lambda_{T}}{M_{\tilde{W}}}-\frac{g^{\prime} \lambda_{S}}{M_{B}}\right) \mu_{A}+\left(\frac{g^{\prime} v_{A}}{M_{B}}\right)^{2} m_{S}+\left(\frac{g v_{A}}{M_{\tilde{W}}}\right)^{2} m_{T} \\
m_{A B} & =\frac{v_{A} v_{u}}{\sqrt{2} \mu}\left(\frac{g \lambda_{T}}{M_{\tilde{W}}}-\frac{g^{\prime} \lambda_{S}}{M_{B}}\right) \mu_{B} \\
m_{A C} & =\frac{v_{A} v_{u}}{\sqrt{2} \mu}\left(\frac{g \lambda_{T}}{M_{\tilde{W}}}-\frac{g^{\prime} \lambda_{S}}{M_{B}}\right) \mu_{C}
\end{aligned}
$$

which has indeed just a zero eigenvalue. 
Let us first of all discuss the upper bound on the gravitino mass, $m_{3 / 2}<0.5$ $\mathrm{GeV}$, imposed by the condition $m_{\nu} \lesssim 0.6 \mathrm{eV}$. Looking at the non zero entries of the mass matrix we see that in general the upper bound depends on the value of $\lambda_{T, S}$. As in the AMRB case, we focus on the $m_{A A}$ entry. When the first term is negligible, the inverse see-saw term gives an upper bound $m_{3 / 2} \lesssim 1-10 \mathrm{keV}$ for $M_{W} \simeq 1 \mathrm{TeV}$ and $v_{A} \lesssim 100 \mathrm{GeV}$. On the other hand, when the first term cannot be neglected, it dominates over the term coming from the inverse see-saw, and the upper bound now reads $m_{3 / 2} \lesssim \frac{0.1 \mathrm{keV}}{\lambda_{S, T}}$ which can be more stringent than in the previous case (depending on the value of $\lambda_{T, S}$ ). We conclude that, under these assumptions, in PMRB the upper bound on the gravitino mass can be significantly lower than the one of the AMRB scenario.

Let us now explain why in this case fitting neutrino physics calls for the introduction of a new sector in the model. Inspecting the phenomenological mass matrices of Eq. 5.3, we see that both hierarchies require leading order entries in the $\mu-\tau$ sector, which cannot be accommodated by the mass matrix (5.15). This is true for any choice of the flavor $A$. At the same time, we expect loop factors to be much smaller than the tree level entries, so that the overall picture cannot be modified too much. This calls for the introduction of a new sector in the model. We can wonder what is the minimal sector able generate neutrino masses and mixing. First of all we would like to generate neutrino physics without the need for a new source of R-breaking. This means we should consider a mechanism that generates neutrino masses and mixing when the lepton number is broken at very low scale (the keV gravitino mass). The minimal possibility we can think of is an inverse see-saw mechanism with additional electroweak singlets ${ }^{3}$.

Therefore, we introduce a right handed Dirac neutrino (two singlets $S$ and $\bar{S}$ with

\footnotetext{
[2].

${ }^{3}$ In general, such Singlets may be present in the sector that generates the hard Yukawa coupling
} 
$R=0$ and $R=2$ respectively) and the following terms in the superpotential:

$$
W=\sum_{i} \lambda_{i} S H_{u} L_{i}+M_{S} S \bar{S}
$$

Each singlet gets a Majorana mass of order of the gravitino mass through Rbreaking effects, and this generates a Majorana neutrinos mass of order $m_{\nu} \sim \frac{\lambda_{i} v_{u}}{M_{S}} m_{S}$. An interesting possibility for the Dirac mass $M_{S}$ is the $\mathrm{TeV}$ scale, since this opens up a link between neutrino physics and LHC physics; however, a complete analysis of this situation is beyond the scope of the thesis and we defer it to a future work.

\subsection{Conclusions}

Neutrino physics can be a natural probe into new physics, and it is then natural to ask whether or not, given a specific framework, neutrino masses and mixing can be accommodated. In this chapter we have studied a supersymmetric scenario where a continuous R-symmetry is identified with the total Lepton Number, so that a possible connection to neutrino physics is immediate. In particular, we have found that neutrino physics is strongly connected with the mechanism of R-symmetry breaking, which in turn is related to supersymmetry breaking.

When R-symmetry breaking effects are communicated to the visible sector solely via Anomaly Mediation, all neutrinos acquire mass at 1-loop level. The hierarchy that can be reproduced depends crucially on the flavor of the sneutrino that gets a vev and plays the role of down type Higgs. For small values of the lightest neutrino mass, and for $A=e$, the case of Normal Hierarchy is disfavored, since it can be reproduced only in a very limited portion of the parameter space. On the contrary, for $A=\mu$, both hierarchies can be fitted in a consistent portion of parameter space. Finally, for $A=\tau$, we are not able to reproduce neutrino phenomenology solely via loop effects. The situation changes increasing the lightest neutrino mass, since in this case both 
hierarchies can be accommodated for $A=e$ and $A=\mu$ (but not for $A=\tau$ ), but only in a limited region of parameter space.

Another possibility is that R-breaking effects are communicated to the visible sector at the Planck scale. In this case two non vanishing neutrino masses are generated at tree level, but with a pattern that does not allow to reproduce the phenomenological matrices studied. Since loop effects give subdominant contributions and cannot change the overall picture, we conclude that a new sector must be added to the theory in order to reproduce neutrino physics. The minimal possibility is to introduce additional singlets (that can however be already present in the sector that generates the hard Yukawa couplings) in order to have an inverse see-saw mechanism. The study of this possibility is however beyond the scope of this thesis.

Since neutrino physics selects a particular region of the parameter space of the model, some consequences on dark matter and collider physics can be inferred. The cosmological upper bound on the sum of neutrino masses translates into an upper bound on the gravitino mass, $m_{3 / 2} \lesssim 0.5 \mathrm{GeV}$ for AMRB (with a more precise range selected by the neutrino mass matrix fit, $\left.m_{3 / 2} \simeq 1 \mathrm{MeV}-100 \mathrm{MeV}\right)$ ), and $m_{3 / 2} \lesssim 10 \mathrm{keV}$ for PMRB. In both scenarios the gravitino lifetime is long enough to evade all experimental bounds, so that it can be a dark matter candidate.

Furthermore, neutrino physics selects also a preferred order of magnitude for the trilinear couplings both in the lepton and quark sector (with the general indication that the off diagonal couplings are larger that the diagonal ones). This can have important consequences for LHC physics. Indeed, one can expect squarks generation changing decays (as $\tilde{b}_{L} \rightarrow \nu_{B} s_{R}$ or $\tilde{t}_{L} \rightarrow e_{B}^{+} s_{R}$ ) to dominate over the corresponding generation conserving decays $\left(\tilde{b}_{L} \rightarrow \nu_{B} b_{R}\right.$ or $\left.\tilde{t}_{L} \rightarrow e_{B}^{+} b_{R}\right)$. A similar conclusion applies in the slepton sector, with decays like $\tilde{\nu}_{B} \rightarrow b \bar{s}$ or $\tilde{e}_{B} \rightarrow s \bar{t}$ generally dominating over $\tilde{\nu}_{B} \rightarrow b \bar{b}$ or $\tilde{e}_{B} \rightarrow b \bar{t}$. 


\section{Chapter 6}

\section{$U(1)_{R}$ lepton number at the $\mathbf{L H C}$}

In this chapter we discuss the main aspects of the LHC phenomenology associated to the supersymmetric model introduced in Chapter 4.

\subsection{Sparticle decay modes}

We start by discussing the decay modes of the sparticles relevant for the LHC collider phenomenology. We make a number of simplifying (but reasonable) assumptions on the sparticle spectrum, which allow us to reduce the parameter space of the model. A more exhaustive exploration of the full parameter space is left to future work.

An important motivation for the assumption that gauginos are Dirac fermions is that it allows the gluinos to be naturally heavier than in the MSSM, as we have

explained in Chapter 4. To be specific, in the following we assume $M_{\tilde{g}}=2 \mathrm{TeV}$, which is sufficiently heavy that direct (pair or associated) gluino production plays a secondary role during the 7-8 TeV run of the LHC. Also, as was established in the previous chapter, the bounds on the electron-chargino mixing require the wino to be heavier than about $1 \mathrm{TeV}$; we will fix its mass at $1.5 \mathrm{TeV}$. The bino mass is not constrained a priori, but it may be natural for it to be lighter than the wino, although likely around the same scale. Our choice will be $M_{\tilde{B}}=1000 \mathrm{GeV}$. 
Since the (electron) sneutrino plays the role of the down-type Higgs, naturalness requires its soft mass to be very close to the electroweak scale. In addition, one does not expect extremely large mass splittings amongst the three generation sleptons/sneutrinos, and therefore we assume that all of them have soft masses of order $v=246 \mathrm{GeV}$. Since the $\mu$-term is also directly connected to the EWSB sector, we also take it to be around $v$. To be definite, we take $\mu=200 \mathrm{GeV}$ and

$m_{\tilde{L}}^{2} \sim m_{\tilde{E}}^{2} \sim(300 \mathrm{GeV})^{2}$ (we will discuss the possibility of finer mass splittings among sleptons later on). With this choice of parameters the lightest chargino/neutralino will be Higgsino-like.

Finally, as long as one focuses on branching fractions, as we do in this section, the precise squark masses do not play a crucial role, provided they are sufficiently heavy (as we will see, current constraints require them to be above $600-800 \mathrm{GeV}$ ). Nevertheless, we assume that $m_{\tilde{q}}<M_{\tilde{B}}$. This is a simplifying assumption since for $m_{\tilde{q}}>M_{\tilde{B}}$ new decay modes (e.g. involving a second lightest neutralino) would open up. Thus, our benchmark scenario is characterized by gauginos heavier than all the sfermions.

We also note that the couplings $\lambda_{S}$ and $\lambda_{T}$ are taken to be of order one, since they may play a crucial role in lifting the SM-like Higgs mass to $\sim 125 \mathrm{GeV}$. To be concrete, we take $\lambda_{S}=\lambda_{T}=0.8$. These couplings affect the neutralino/chargino composition and are therefore relevant for the collider phenomenology. The singlet, triplet and octet scalars are taken to be in the multi-TeV range so that they play a secondary role that will not be studied here (for studies of the octet scalar phenomenology, see [116].

\subsubsection{Neutralino and Chargino decay modes}

The model contains four massive Dirac charginos, three massive Dirac neutralinos and a massless (Weyl) neutralino. The lightest of the four charginos corresponds to the physical electron, while the massless neutralino is the electron-neutrino. In 
this subsection we focus on the decay modes of the lightest "standard" (i.e. not the neutrino or electron) neutralino $\tilde{X}_{1}^{0}$ and chargino $\tilde{X}_{1}^{+-} / \tilde{X}_{1}^{-+1}$. These are the most relevant ones for the LHC phenomenology.

\section{Neutralino sector:}

The three physical neutralinos are linear combinations of the bino $(\tilde{B})$, singlino $(\tilde{S})$, wino $(\tilde{W})$, neutral tripletino $\left(\tilde{T}^{0}\right)$, the up- and down-type higgsinos $\left(\tilde{h}_{u}\right.$ and $\left.\tilde{h}_{d}\right)$ and the "electron-neutrino" $\left(\nu_{e}\right)$. In two-component notation, we have neutralino states of definite $U(1)_{R}$ charge

$$
\begin{aligned}
& \tilde{\chi}_{i}^{0+}=V_{i \tilde{b}}^{N} \tilde{B}+V_{i \tilde{w}}^{N} \tilde{W}+V_{i d}^{N} \tilde{h}_{d}^{0}, \\
& \tilde{\chi}_{i}^{0-}=U_{i \tilde{s}}^{N} \tilde{S}+U_{i \tilde{t}}^{N} \tilde{T}^{0}+U_{i u}^{N} \tilde{h}_{u}^{0}+U_{i \nu}^{N} \nu_{e}
\end{aligned}
$$

where $V_{i k}^{N}$ and $U_{i k}^{N}$ are the unitary matrices that diagonalize the neutralino mass matrix. These states form Dirac fermions $\tilde{X}_{i}^{0}=\left(\tilde{\chi}_{i}^{0+}, \bar{\chi}^{0-}{ }_{i}\right)$, for $i=1,2,3$. In addition, there remains a massless Weyl neutralino:

$$
\tilde{\chi}_{4}^{0-}=U_{4 \tilde{s}}^{N} \tilde{S}+U_{4 \tilde{t}}^{N} \tilde{T}^{0}+U_{4 u}^{N} \tilde{h}_{u}^{0}+U_{4 \nu}^{N} \nu_{e}
$$

which corresponds to the physical electron-neutrino. With some abuse of notation we will refer to it as " $\nu_{e}$ " in the following, where it will alway refer to the above mass eigenstate and should cause no confusion with the original gauge eigenstate. The mixing in the neutrino sector associated to neutrino oscillations plays no role in our discussion, and will be neglected throughout.

Although -given the assumptions spelled out in the previous subsection- the lightest neutralino $\tilde{X}_{1}^{0}$ is mostly higgsino, its smaller wino and bino components can play an important role. In the region of parameter space we are focusing on, these components depend most crucially on the $\lambda_{S}$ and $\lambda_{T}$ couplings, while being insensitive to the sneutrino vev. We illustrate this in Fig. 6.1. We consider two possibilities for the

\footnotetext{
${ }^{1}$ In this chapter we will adopt a slightly different notation for chargino and neutralino where the two upper indices indicate the electric and the $R$ charge respectively
} 

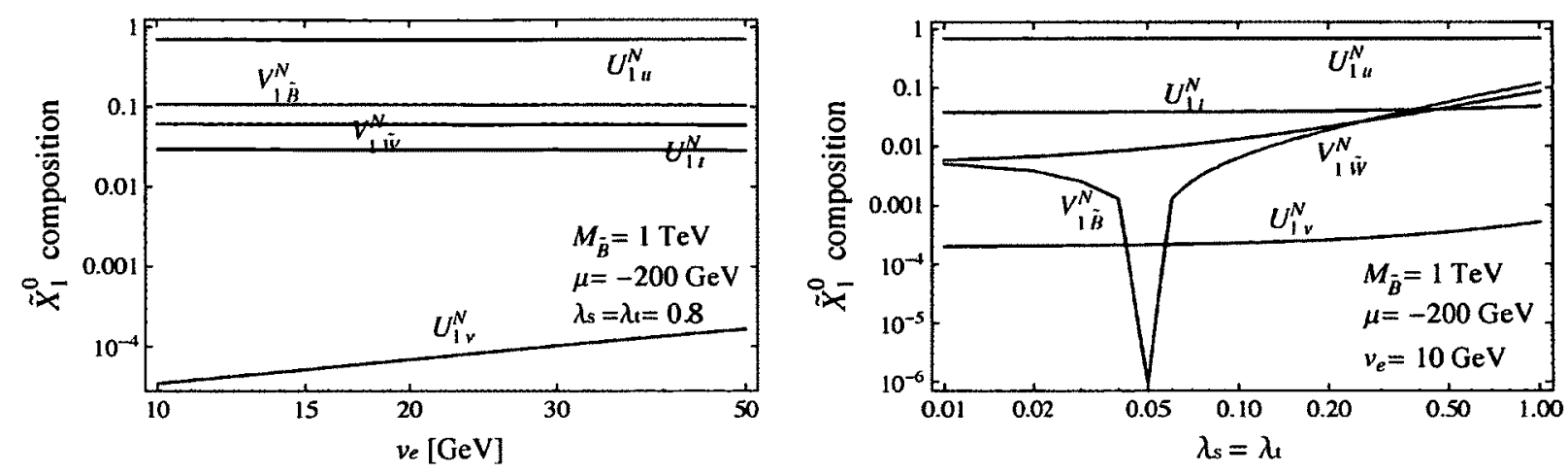

Figure 6.1: $\tilde{X}_{1}^{0}$ mixing angles for $M_{\tilde{W}}=1.5 \mathrm{TeV}, M_{\tilde{B}}=800 \mathrm{GeV}$ and $\mu=200 \mathrm{GeV}$, exhibiting the dependence on $\lambda_{S}, \lambda_{T}$ and $v_{e}$. The plot on the left is for fixed $\lambda_{S}=$ $\lambda_{T}=0.8$, while the one on the right is for fixed $v_{e}=10 \mathrm{GeV}$. We always plot the absolute magnitude of the rotation matrix elements, $V_{i k}^{N}$ and $U_{i k}^{N}$.

LSP : it could either be the "standard" lightest neutralino, $\tilde{X}_{1}^{0}$, or the left handed (LH) stau, $\tilde{\tau}_{L}$. The decay modes of the lightest neutralino depend on this choice and we will consider them separately.

If $\tilde{X}_{1}^{0}$ is lighter than $\tilde{\tau}_{L}$, the possible decay modes for $\tilde{X}_{1}^{0}$ have partial decay widths:

$$
\begin{aligned}
\Gamma\left(\tilde{X}_{1}^{0} \rightarrow W e\right)= & \frac{g^{2} m_{\tilde{X}_{1}^{0}}}{64 \pi}\left(U_{1 \nu}^{N} U_{4 \nu}^{N}+\sqrt{2} U_{1 \tilde{t}}^{N} U_{4 \tilde{t}}^{N}\right)^{2}\left(1-\frac{M_{W}^{2}}{m_{\tilde{X}_{1}^{0}}^{2}}\right)^{2}\left(2+\frac{m_{\tilde{X}_{1}^{0}}^{2}}{M_{W}^{2}}\right) \\
\Gamma\left(\tilde{X}_{1}^{0} \rightarrow Z \nu_{e}\right)= & \frac{g^{2} m_{\tilde{X}_{1}^{0}}}{128 \pi c_{W}^{2}}\left(U_{1 \nu}^{N} U_{4 \nu}^{N}-U_{1 u}^{N} U_{4 u}^{N}\right)^{2}\left(1-\frac{M_{Z}^{2}}{m_{\tilde{X}_{1}^{0}}^{2}}\right)^{2}\left(2+\frac{m_{\tilde{X}_{1}^{0}}^{2}}{M_{Z}^{2}}\right) \\
\Gamma\left(\tilde{X}_{1}^{0} \rightarrow h \nu_{e}\right)= & \frac{m_{\tilde{X}_{1}^{0}}}{64 \pi}\left(1-\frac{m_{h}^{2}}{m_{\chi_{1}^{0}}^{2}}\right)^{2} \times \\
& {\left[\left(-g V_{1 \tilde{w}}^{N} U_{4 u}^{N}+g^{\prime} V_{1 \bar{b}}^{N} U_{4 u}^{N}\right) R_{1 u}+\left(g V_{1 \tilde{w}}^{N} U_{4 \nu}^{N}-g^{\prime} V_{1 \tilde{b}}^{N} U_{4 \nu}^{N}\right) R_{1 \tilde{\nu}}\right.} \\
& \left.+\sqrt{2}\left(\lambda_{S} V_{1 d}^{N} U_{4 \tilde{s}}^{N}+\lambda_{T} V_{1 d}^{N} U_{4 \tilde{t}}^{N}\right) R_{1 u}+\sqrt{2} V_{1 d}^{N} U_{4 u}^{N}\left(\lambda_{S} R_{1 s}+\lambda_{T} R_{1 t}\right)\right]^{2},
\end{aligned}
$$

where $R_{1 i}$ are the mixing angles characterizing the composition of the lightest Higgs, $h$. In our benchmark region all the other Higgs bosons are heavier than the lightest neutralino. The above decay modes can easily be dominated by the neutrino-neutralino mixing angle, since the contributions due to the higgsino and 
tripletino/singlino components are highly suppressed. This mixing angle, in turn, is controlled by the sneutrino vev. Note that in the RPV MSSM such decay modes are typically characterized by displaced vertices due to the stringent bounds on the sneutrino vev arising from neutrino physics, [118], [119]. By contrast, in our scenario the sneutrino vev is allowed to be sizable (10's of $\mathrm{GeV})$, and is in fact bounded from below from perturbativity/EWPT arguments, so that these decays are prompt. The decay width into $Z \nu_{e}$ is the dominant one in the large sneutrino vev limit, while in the small sneutrino vev limit the dominant neutralino decay mode is $h \nu_{e}$, as shown in Fig. 6.1.1. Note also that the $W e$ channel is suppressed compared to the $Z \nu_{e}$ one. This is a result of a partial cancellation between the mixing angles in Eq. (6.5). The pattern for the lightest neutralino decay modes we just described dominates the parameter space of the model. However, there is large portion of the parameter space where the decay mode into $W e$ is important and it could even be the dominant decay mode as it is shown in Fig. 6.3. The benchmark point corresponding to Fig. 6.3 has $\mu=200 \mathrm{GeV}$ and $\lambda_{S}=\lambda_{T}=0.4$. For these values the cancellation between the mixing angles is such $Z \nu_{e}$ is suppressed over $W e$. In the left panel of Fig. 6.3 we computed the magnitude of the neutralino mixing angle for this different choice of parameters.

If instead the $\tilde{\tau}_{L}$ is the LSP, the $\tilde{\tau}_{L} \tau$ and $\tilde{\nu}_{\tau} \nu_{\tau}$ channels open up with a partial decay width given by

$$
\begin{aligned}
\Gamma\left(\tilde{X}_{1}^{0} \rightarrow \tilde{\tau}_{L} \tau\right) & =\frac{3 g^{2}}{32 \pi}\left(V_{1 \tilde{w}}^{N}+\tan \theta_{W} V_{1 \tilde{b}}^{N}\right)^{2} m_{\tilde{X}_{1}^{0}}\left(1-\frac{m_{\tilde{\tau}_{L}}^{2}}{m_{\tilde{X}_{1}^{0}}^{2}}\right)^{2} \\
\Gamma\left(\tilde{X}_{1}^{0} \rightarrow \tilde{\nu}_{\tau} \nu_{\tau}\right) & =\frac{3 g^{2}}{32 \pi}\left(V_{1 \tilde{w}}^{N}-\tan \theta_{W} V_{1 \tilde{b}}^{N}\right)^{2} m_{\tilde{X}_{1}^{0}}\left(1-\frac{m_{\tilde{\nu}_{\tau}}^{2}}{m_{\tilde{X}_{1}^{0}}^{2}}\right)^{2}
\end{aligned}
$$



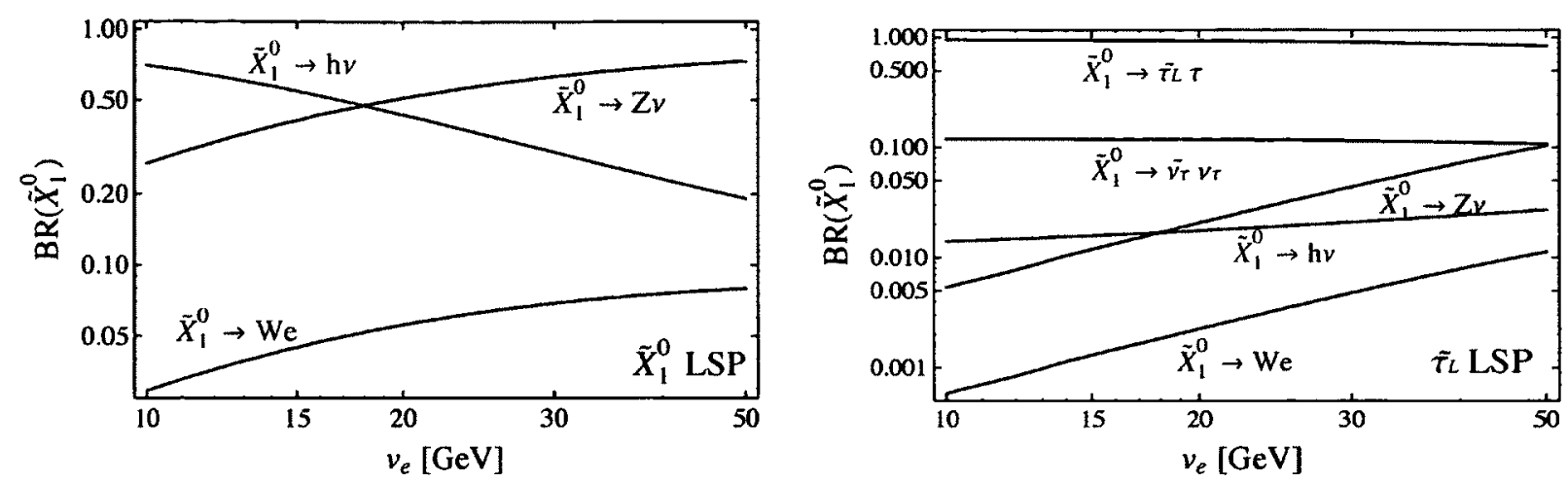

Figure 6.2: $\tilde{X}_{1}^{0}$ decay modes in the case that $\tilde{X}_{1}^{0}$ is the LSP (left panel) and in the case that $\tilde{\tau}_{L}$ is the LSP (right panel).
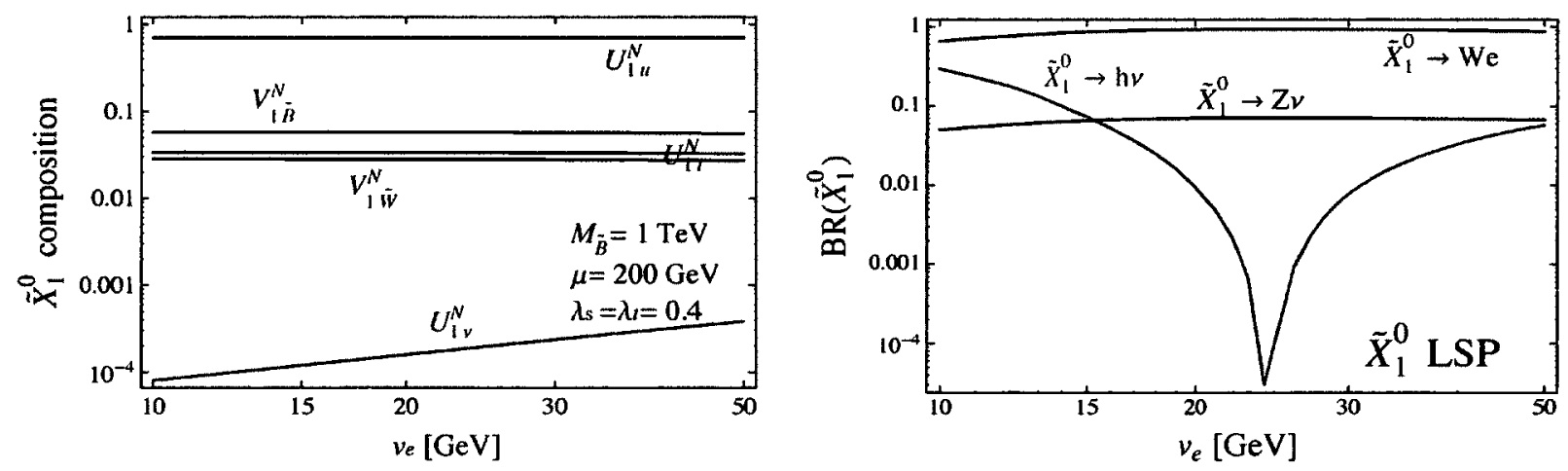

Figure 6.3: $\tilde{X}_{1}^{0}$ mixing angles ( left panel) and branching ratio ( right panel ) for $\tilde{X}_{1}^{0}$ LSP scenario with for $M_{\bar{W}}=1.5 \mathrm{TeV}, M_{\bar{B}}=1 \mathrm{TeV}, \mu=200 \mathrm{GeV}$ and $\lambda_{S}=\lambda_{T}=0.4$.

Note that this depends on the gaugino components only and not on the electron or neutrino fraction. As a result, even for the suppresed $V_{1 \bar{w}}^{N}$ and $V_{1 \tilde{b}}^{N}$ shown in Fig. 6.1 these decay channels dominate over the ones driven by the neutrino-neutralino mixing, as shown in the right panel of Fig. .6.1.1. Also the decay mode into $\tilde{\nu}_{\tau} \nu_{\tau}$ is suppressed compared to the one into the charged lepton and slepton due to a cancellation between the mixing angle in Eq. (6.7).

Chargino sector:

We can split the four Dirac charginos (one of which is the physical electron) according to their electric charge $Q$ and $R$ charges. Again in two-component notation, 
the ones with $R=Q$ are:

$$
\begin{aligned}
& \tilde{\chi}_{i}^{++}=V_{i \bar{w}}^{+} \tilde{W}^{+}+V_{i e}^{+} e_{R}^{c}, \\
& \tilde{\chi}_{i}^{--}=U_{i \tilde{t}}^{+} \tilde{T}_{d}^{-}+U_{i e}^{+} e_{L}^{-},
\end{aligned}
$$

where $V_{i k}^{+}$and $U_{i k}^{+}$are the unitary matrices that diagonalize the chargino mass matrix in the $\left\{\tilde{T}_{d}^{-}, e_{L}^{-}\right\}$versus $\left\{\tilde{W}^{+}, e_{R}^{c}\right\}$ basis. The superscript in the rotation matrices indicates the product $R \times Q$. Recall that $e_{L}^{-}$is the electron $S U(2)$ doublet while $e_{R}^{c}$ is the $S U(2)$ singlet. The subindex labelling the mass eigenstates takes values $i=1,2$. The states with $R=-Q$, are:

$$
\begin{aligned}
& \tilde{\chi}_{i}^{+-}=V_{i \tilde{t}}^{-} \tilde{T}_{u}^{+}+V_{i u}^{-} \tilde{h}_{u}^{+}, \\
& \tilde{\chi}_{i}^{-+}=U_{i \tilde{w}}^{-} \tilde{W}^{-}+U_{i d}^{-} \tilde{h}_{d}^{-} .
\end{aligned}
$$

where now $V_{i k}^{-}$and $U_{i k}^{-}$are the $2 \times 2$ unitary matrices that diagonalize the chargino mass matrix in the $\left\{\tilde{W}^{-}, h_{d}^{-}\right\}$versus $\left\{\tilde{T}_{u}^{+}, h_{u}^{+}\right\}$basis, and again the superscript indicates the product $R \times Q$. All of these states come in degenerate pairs that can be arranged into four 4-component Dirac fields, $\tilde{X}_{i}^{++}=\left(\tilde{\chi}_{i}^{++},{\overline{\tilde{\chi}^{--}}}_{i}\right)$ and $\tilde{X}_{i}^{+-}=$ $\left(\tilde{\chi}_{i}^{+-}, \overline{\tilde{\chi}}^{-+}\right.$), for $i=1,2$. In this notation, $e=\tilde{X}_{1}^{++}$corresponds to the physical electron (Dirac) field. We exhibit the mixing angles in Fig. 6.1.

Since the wino mass is constrained to be above $1 \mathrm{TeV}$ the lightest "standard" chargino is mostly higgsino, which means it has $R=-Q$, while the electron and the other charged leptons have $R=Q$. Therefore, two-body decays of $\tilde{X}_{1}^{+-}$can involve a charged lepton only when accompanied with an electrically neutral, $|R|=2$ particle, the only example of which is the $h_{d}^{0}$ scalar. We assume this state to be heavier than the lightest chargino, ${ }^{2}$ which has important consequences for the allowed chargino decay modes. For instance, in the region where $\tilde{\tau}_{L}$ is heavier than $\tilde{X}_{1}^{+-}$the potentially allowed decay modes of $\tilde{X}_{1}^{+-}$are into $W \nu_{e}$ and $W \tilde{X}_{1}^{0}$. However, the latter is closed in

\footnotetext{
${ }^{2}$ Recall that the $H_{d} S U(2)$ doublet does not play any role in EWSB.
} 
most of the parameter space since $\tilde{X}_{1}^{0}$ and $\tilde{X}_{1}^{+-}$are almost degenerate (with a mass splitting of order $10^{\prime} s \mathrm{GeV}$ ). The dominant decay mode has a partial decay width given by:

$$
\Gamma\left(\tilde{X}_{1}^{+-} \rightarrow W \nu_{e}\right)=\frac{g^{2}}{128 \pi}\left(V_{1 u}^{-} U_{4 u}^{N}-\sqrt{2} V_{1 \bar{t}}^{-} U_{4 \bar{t}}^{N}\right)^{2} m_{\tilde{X}_{1}}\left(1-\frac{M_{W}^{2}}{m_{\tilde{X}_{1}}^{2}}\right)^{2}\left(2+\frac{m_{\tilde{X}_{1}}^{2}}{M_{W}^{2}}(\oint) .13\right)
$$

where we denote the mass of $\tilde{X}_{1}^{+-}$by $m_{\tilde{X}_{1}}$. Therefore, for sufficiently heavy sleptons the chargino always decays into $W \nu_{e}$.

If instead $\tilde{\tau}_{L}$ is lighter than $\tilde{X}_{1}^{+-}$the dominant decay mode becomes $\tilde{X}_{1}^{+-} \rightarrow \tilde{\tau}_{L}^{+} \nu_{\tau}$ which follows by comparing Eq. (6.13) against

$$
\Gamma\left(\tilde{X}_{1}^{+-} \rightarrow \tilde{\tau}_{L}^{+} \nu_{\tau}\right)=\frac{3 g^{2}}{32 \pi}\left(U_{1 \tilde{w}}^{-}\right)^{2} m_{\tilde{X}_{1}}\left(1-\frac{m_{\tilde{\tau}_{L}}^{2}}{m_{\tilde{X}_{1}}^{2}}\right)^{2}
$$

and the mixing angles shown in Fig. 6.1.

\subsubsection{Slepton decay modes}

The sleptons are expected to be among the lightest sparticles in the new physics spectrum. This is due to the intimate connection of the slepton sector with EWSB, together with the fact that a certain degree of degeneracy between the three generation sleptons is expected. The possible exception is the LH third generation slepton doublet, if the RPV Yukawa coupling $\lambda_{333}^{\prime}$ turns out to be sizable (as is currently allowed). As a result, due to RG running, the $\mathrm{LH}$ stau can be several tens of $\mathrm{GeV}$ lighter than the selectron and smuon, while the latter should have masses within a few $\mathrm{GeV}$ of each other. In this section we focus on the decays of $\tilde{\tau}_{L}$ since it may very well be the LSP. Whether the lightest neutralino $\tilde{X}_{1}^{0}$ or $\tilde{\tau}_{L}$ end up being the LSP is controlled by the size of the $\mu$-term relative to the slepton soft masses.

If the lightest neutralino is the LSP the dominant decay modes would be $\tilde{\tau}_{L} \rightarrow \tilde{X}_{1}^{0} \tau$ or $\tilde{\tau}_{L}^{+} \rightarrow \tilde{X}_{1}^{+-} \bar{\nu}_{\tau}$. If instead $\tilde{\tau}_{L}$ is the LSP, its decay modes are $\tilde{\tau}_{L}^{-} \rightarrow e^{-} \bar{\nu}_{\tau}$ and $\tilde{\tau}_{L}^{-} \rightarrow \bar{t} b$. 

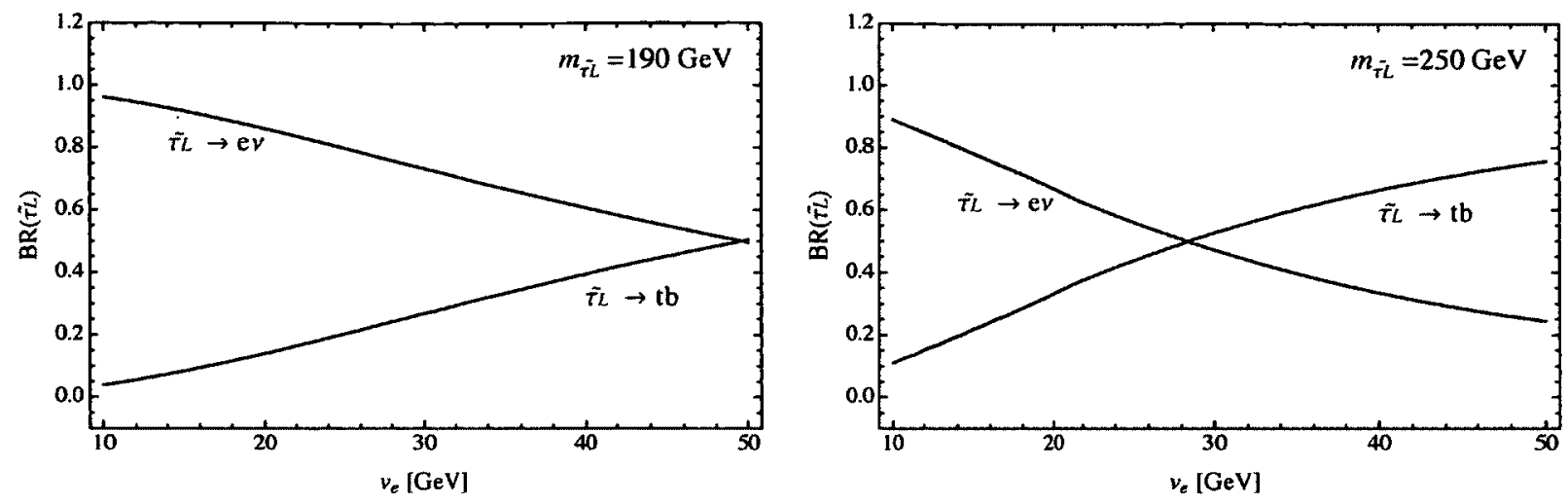

Figure 6.4: $\tilde{\tau}_{L}$ decay modes for two stau masses: $m_{\tilde{\tau}}=190 \mathrm{GeV}$ and $m_{\tilde{\tau}}=250 \mathrm{GeV}$. It is assumed that the lightest neutralino is heavier than the stau.

The corresponding partial decay widths are:

$$
\begin{aligned}
\Gamma\left(\tilde{\tau}_{L}^{-} \rightarrow \tau^{-} \bar{\nu}_{e}\right) & =\frac{m_{\tilde{\tau}_{L}}}{16 \pi} y_{b}^{2} \\
\Gamma\left(\tilde{\tau}_{L}^{-} \rightarrow \bar{t} b\right) & =\frac{m_{\tilde{\tau}_{L}}}{16 \pi}\left(\lambda_{333}^{\prime}\right)^{2}\left(1-\frac{m_{t}^{2}}{m_{\tilde{\tau}_{L}^{2}}}\right) .
\end{aligned}
$$

Since the bounds on the RPV couplings (see Table.(4.5) in Chapter 4) imposes $\lambda_{333}^{\prime}=\left(1.8 \times 10^{-2}\right) / y_{b}$, where $y_{b}=m_{b} / v_{e}$, we expect the decay mode into $\bar{t} b$ to be dominant in the large sneutrino vev/small $\tan \beta$ limit, where $y_{b}$ is smallest. Note that, using $m_{b} \approx 2.8 \mathrm{GeV}$, we have $\lambda_{333}^{\prime} \approx 1.1 \cos \beta$, which can be of order one. However, the $\bar{t} b$ decay mode may be suppressed by phase space when $m_{\tilde{\tau}_{L}} \sim m_{t}$ as shown in Fig. 6.4.

\subsubsection{Squark decay modes}

As already explained, we focus on the case where the gluinos are heavier than the squarks and, therefore, the squark decay mode into a gluino plus jet is kinematically closed. The lightest neutralinos and charginos are instead expected to be lighter than the squarks since naturalness requires the $\mu$-term to be at the electroweak scale. Thus, 
the squark decays into a quark plus the lightest neutralino or into a quark plus the lightest chargino should be kinematically open. However, the decay mode of the left handed up-type squarks, which have $Q=2 / 3$ and $R=1$, into the lightest chargino $\tilde{X}_{1}^{+-}$plus a $R$-neutral jet is forbidden by the combined conservation of the electric and R-charges: $\tilde{u}_{L} \nrightarrow \tilde{X}_{1}^{+-} j$. The decay mode into the second lightest neutralino, which can be of the $(++)$ type, could be allowed by the quantum numbers, but our choice $M_{\tilde{B}}>m_{\tilde{q}}$ ensures that it is kinematically closed. Note also that since $u_{R}$ has $Q=2 / 3$ and $R=-1$, one can have $\tilde{u}_{R} \rightarrow \tilde{X}_{1}^{+-} j$.

Furthermore, there are lepto-quark decay modes for the left-handed squarks and the down-type right-handed squarks, via the down-type Yukawa couplings, $\lambda_{1 k k}^{\prime}$, and the other RPV interactions $\lambda^{\prime}$ (and $\lambda$ ). Most of these couplings are vey suppressed for the first and second generations, and in the following we will keep just the downtype Yukawa couplings which, although small, can give rise to subdominant, but potentially interesting signatures. In contrast, the third generation Yukawa couplings are sizable and can therefore have an important impact on the third generation squark branching fractions. Furthermore, the coupling $\lambda_{333}^{\prime} \lesssim 1.8 \times 10^{-2}\left(v_{e} / m_{b}\right)$ can be of the order of $g^{\prime}$ in the large vev limit, so that for the third generation there may be several lepto-quark channels with sizable branching fractions.

\section{First and second generation squarks}

- The left-handed up-type squarks, $\tilde{u}_{L}$ and $\tilde{c}_{L}$, decay into $\tilde{X}_{1}^{0} j$ and $e j$ with:

$$
\begin{aligned}
\Gamma\left(\tilde{u}_{L} \rightarrow \tilde{X}_{1}^{0} j\right) & \left.=\frac{m_{\tilde{q}}}{16 \pi}\left[\frac{1}{18}\left(g^{\prime} V_{1 \tilde{b}}^{N}+3 g V_{1 \tilde{w}}^{N}\right)^{2}+2 y_{u}^{2}\left(U_{1 u}^{N}\right)^{2}\right]\left(1-\frac{m_{\tilde{X}_{1}^{0}}^{2}}{m_{\tilde{q}}^{2}}\right) 6.17\right) \\
\Gamma\left(\tilde{u}_{L} \rightarrow e_{L}^{+} j\right) & =\frac{m_{\tilde{q}}}{16 \pi} y_{d}^{2}\left(U_{1 e}^{+}\right)^{2}
\end{aligned}
$$

and analogous expressions for $\tilde{c}_{L}$. Taking into account the smallness of the Yukawa couplings for the first two generations, together with the $\tilde{X}_{1}^{0}$ composition shown in Fig. 6.1, one finds that the dominant decay mode is the one into 
neutralino and a jet. Therefore, in the region of parameter space we are interest in, $\tilde{u}_{L}$ and $\tilde{c}_{L}$ decay into $\tilde{X}_{1}^{0} j$ with almost $100 \%$ probability.

- The down-type left-handed squarks, $\tilde{d}_{L}$ and $\tilde{s}_{L}$, have the following decay channels:

$$
\begin{aligned}
\Gamma\left(\tilde{d}_{L} \rightarrow \tilde{X}_{1}^{0} j\right) & =\frac{m_{\tilde{q}}}{16 \pi}\left[\frac{1}{18}\left(g^{\prime} V_{1 \tilde{b}}^{N}-3 g V_{1 \tilde{w}}^{N}\right)^{2}+2 y_{u}^{2}\left(U_{1 u}^{N}\right)^{2}\right]\left(1-\frac{m_{\tilde{X}_{1}^{0}}^{2}}{m_{\tilde{q}}^{2}}\right) \\
\Gamma\left(\tilde{d}_{L} \rightarrow \tilde{X}_{1}^{-+} j\right) & =\frac{m_{\tilde{q}}}{16 \pi}\left[g^{2}\left(U_{1 \tilde{w}}^{-}\right)^{2}+2 y_{u}^{2}\left(V_{1 u}^{-}\right)^{2}\right]\left(1-\frac{m_{\tilde{X}_{1}}^{2}}{m_{\tilde{q}}^{2}}\right) \\
\Gamma\left(\tilde{d}_{L} \rightarrow \bar{\nu}_{e} j\right) & =\frac{m_{\tilde{q}}}{16 \pi}\left(U_{4 \nu}^{N}\right)^{2} y_{d}^{2}
\end{aligned}
$$

with analogous expressions for $\tilde{s}_{L}$. The relative minus sign in the gaugino contributions to the neutralino decay channel is due to the $S U(2)$ charge of the down-type squarks, and should be compared to the up-type case, Eq. (6.17). This leads to a certain degree of cancellation between the contributions from the bino and wino components, which together with the factor of $1 / 18$ results in a significant suppression of the neutralino channel. Since the Yukawa couplings are very small, it follows that the chargino channel is the dominant decay mode of the down-type squarks of the first two generations.

- The right-handed up-type squarks, $\tilde{u}_{R}$ and $\tilde{c}_{R}$, decay according to

$$
\begin{aligned}
\Gamma\left(\tilde{u}_{R} \rightarrow \tilde{X}_{1}^{0} j\right) & =\frac{m_{\tilde{q}}}{36 \pi}\left(g^{\prime} V_{1 \tilde{B}}^{N}\right)^{2}\left(1-\frac{m_{\tilde{X}_{1}^{0}}^{2}}{m_{\tilde{q}}^{2}}\right), \\
\Gamma\left(\tilde{u}_{R} \rightarrow \tilde{X}_{1}^{+-} j\right) & =\frac{m_{\tilde{q}}}{8 \pi}\left(y_{u} V_{1 u}^{-}\right)^{2} .
\end{aligned}
$$

The chargino decay mode is always suppressed since the Yukawa couplings are very small. Therefore, the right-handed up-type squarks decay into $\tilde{X}_{1}^{0} j$ with almost $100 \%$ probability. 
- The right-handed down-type squarks, $\tilde{d}_{R}$ and $\tilde{s}_{R}$, decay according to

$$
\begin{aligned}
\Gamma\left(\tilde{d}_{R} \rightarrow \tilde{X}_{1}^{0} j\right) & =\frac{m_{\tilde{q}}}{16 \pi} \frac{\left(g^{\prime} V_{1 \tilde{B}}^{N}\right)^{2}}{9}\left(1-\frac{m_{\tilde{X}_{1}^{0}}^{2}}{m_{\tilde{q}}^{2}}\right), \\
\Gamma\left(\tilde{d}_{R} \rightarrow e_{L}^{-} j\right) & =\frac{m_{\tilde{q}}}{16 \pi}\left(U_{1 e}^{+}\right)^{2} y_{d}^{2}, \\
\Gamma\left(\tilde{d}_{R} \rightarrow \nu_{e} j\right) & =\frac{m_{\tilde{q}}}{16 \pi}\left(U_{4 \nu}^{N}\right)^{2} y_{d}^{2} .
\end{aligned}
$$

Since the Yukawa couplings are very small these squarks decay dominantly into neutralino plus jet.

In summary, for the first and second generation squarks the lepto-quark signals are not easily visible due to the smallness of the Yukawa couplings, and the fact that the gaugino mixing angles are sufficiently large (even though $\tilde{X}_{1}^{0}$ and $\tilde{X}_{1}^{+-}$are higgsinolike) for the phenomenology to be controlled by the gauge interactions. We note, however, that for very small $\lambda_{S}$ and $\lambda_{T}$ we can have a sizable branching fraction for the second generation lepto-quark signals. ${ }^{3}$ Indeed, as shown in the right plot of Fig. 6.1, the gaugino content shows a significant dependence on $\lambda_{S}$ and $\lambda_{T}$, and can be rather suppressed when these become very small. It would be interesting to explore the phenomenology of such a case, but for the moment we focus on the $\lambda_{S} \sim \lambda_{T} \sim \mathcal{O}(1)$ region.

\section{Third generation}

For the third generation we expect the lepto-quark signals to be visible in all of our parameter space, although they may be of different types. The point is that the bottom Yukawa coupling can be sizable in the small sneutrino vev/large $\tan \beta$ limit (as in the MSSM), thus leading to a signal involving first generation leptons through the $\lambda_{133}^{\prime} \equiv y_{b} \approx 1.16 \times 10^{-2} \sec \beta$ coupling. In the large sneutrino vev/small $\tan \beta$

\footnotetext{
${ }^{3}$ In this case lifting the SM-like Higgs mass to $125 \mathrm{GeV}$ would presumably require additional physics.
} 
limit, on the other hand, the RPV coupling $\lambda_{333}^{\prime} \lesssim 1.1 \cos \beta$ can be of order of $g^{\prime}$, and may lead to third generation leptons in the final state.

- The left-handed stop, $\tilde{t}_{L}$, has the following decay modes:

$$
\begin{aligned}
\Gamma\left(\tilde{t}_{L} \rightarrow \tilde{X}_{1}^{0} t\right) & =\frac{m_{\tilde{t}_{L}}}{16 \pi}\left[\frac{1}{18}\left(g^{\prime} V_{1 \tilde{b}}^{N}+3 g V_{1 \tilde{w}}^{N}\right)^{2}+2 y_{t}^{2}\left(U_{1 u}^{N}\right)^{2}\right] f_{P H}\left(m_{\tilde{t}_{L}}, m_{\tilde{X}_{1}^{0}}, m_{t}\right) \\
\Gamma\left(\tilde{t}_{L} \rightarrow e_{L}^{+} b\right) & =\frac{m_{\tilde{t}_{L}}}{16 \pi} y_{b}^{2}\left(U_{1 e}^{+}\right)^{2} \\
\Gamma\left(\tilde{t}_{L} \rightarrow \tau_{L}^{+} b\right) & =\frac{m_{\tilde{t}_{L}}}{16 \pi}\left(\lambda_{333}^{\prime}\right)^{2}
\end{aligned}
$$

where the phase space factor is defined as:

$$
f_{P H}\left(m_{1}, m_{2}, m_{3}\right)=\sqrt{1+\frac{m_{2}^{4}}{m_{1}^{4}}+\frac{m_{3}^{4}}{m_{1}^{4}}-\left(\frac{m_{2}^{2}}{m_{1}^{2}}+\frac{m_{3}^{2}}{m_{1}^{2}}+\frac{m_{2}^{2} m_{3}^{2}}{m_{1}^{4}}\right)} .
$$

The decay mode into neutralino plus top is the dominant one since it is driven by the top Yukawa coupling, as shown in Fig. 6.5. However, this figure also shows that the two lepto-quark decay modes can have sizable branching fractions. In particular, at small sneutrino vev the electron-bottom channel is the dominant lepto-quark decay mode (since it is proportional to the bottom Yukawa), while in the large vev limit the third generation lepto-quark channel dominates (we have taken $\lambda_{333}^{\prime}=\left(1.8 \times 10^{-2}\right) / y_{b}$, thus saturating the upper bound arising from EWPT). The existence of lepto-quark channels with a sizable (but somewhat smaller than one) branching fraction is a distinctive feature of our model, as will be discussed in more detail in the following section. 


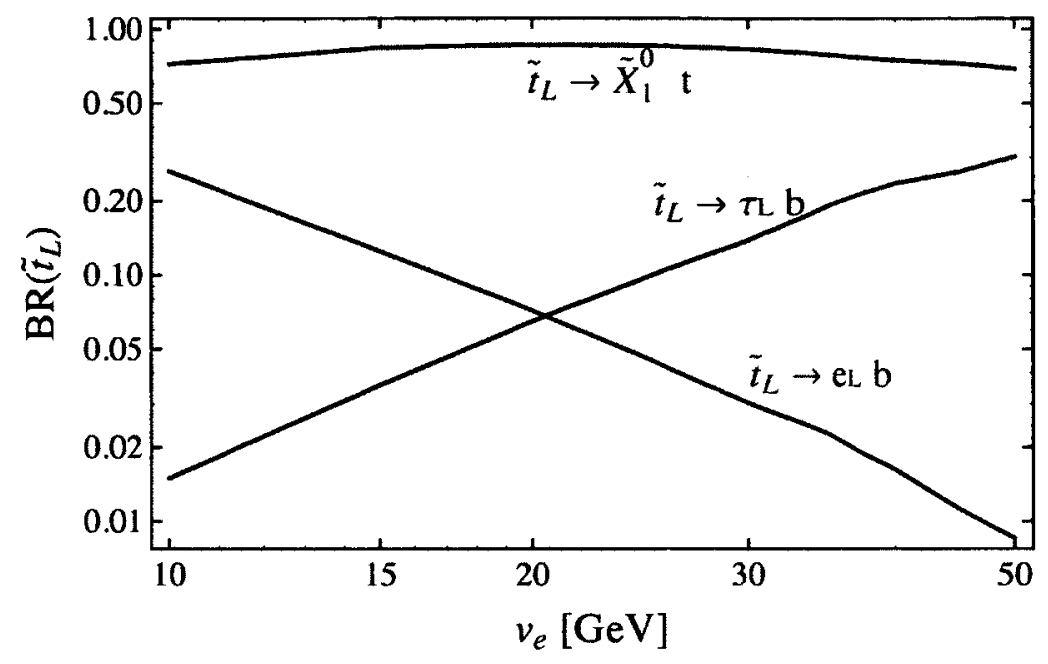

Figure 6.5: Branching fractions for the $\tilde{t}_{L}$ decay modes computed for $\lambda_{S}=\lambda_{T}=0.8$, $\mu=200 \mathrm{GeV}, M_{\tilde{B}}=800 \mathrm{GeV}$ and $M_{\tilde{W}}=1500 \mathrm{GeV}$. We also take $\lambda_{333}^{\prime}=(1.8 \times$ $\left.10^{-2}\right) / y_{b}$.

- The left-handed sbottom, $\tilde{b}_{L}$, has several decay modes as follows:

$$
\begin{aligned}
\Gamma\left(\tilde{b}_{L} \rightarrow \tilde{X}_{1}^{0} b\right) & =\frac{m_{\tilde{b}_{L}}}{16 \pi}\left[\frac{1}{18}\left(g^{\prime} V_{1 \tilde{b}}^{N}-3 g V_{1 \tilde{w}}^{N}\right)^{2}+2 y_{t}^{2}\left(U_{1 u}^{N}\right)^{2}\right]\left(1-\frac{m_{\tilde{X}_{1}^{0}}^{2}}{m_{\tilde{b}_{L}}^{2}}\right) \\
\Gamma\left(\tilde{b}_{L} \rightarrow \tilde{X}_{1}^{-+} t\right) & =\frac{m_{\tilde{b}_{L}}}{16 \pi}\left[g^{2}\left(U_{1 \tilde{w}}^{-}\right)^{2}+2 y_{t}^{2}\left(V_{1 u}^{-}\right)^{2}\right] f_{P H}\left(m_{\tilde{b}_{L}}, m_{\tilde{X}_{1}^{ \pm}}, m_{t}\right) \\
\Gamma\left(\tilde{b}_{L} \rightarrow \bar{\nu}_{e} b\right) & =\frac{m_{\tilde{b}_{L}}}{16 \pi}\left(U_{4 \nu}^{N}\right)^{2} y_{b}^{2} \\
\Gamma\left(\tilde{b}_{L} \rightarrow \bar{\nu}_{\tau} b\right) & =\frac{m_{\tilde{b}_{L}}}{16 \pi}\left(\lambda_{333}^{\prime}\right)^{2}
\end{aligned}
$$

The dominant decay mode is into a chargino plus top since it is controlled by the top Yukawa coupling. The decays into neutrino and bottom have always a sizable branching fraction, as can be seen in Fig. 6.6. 


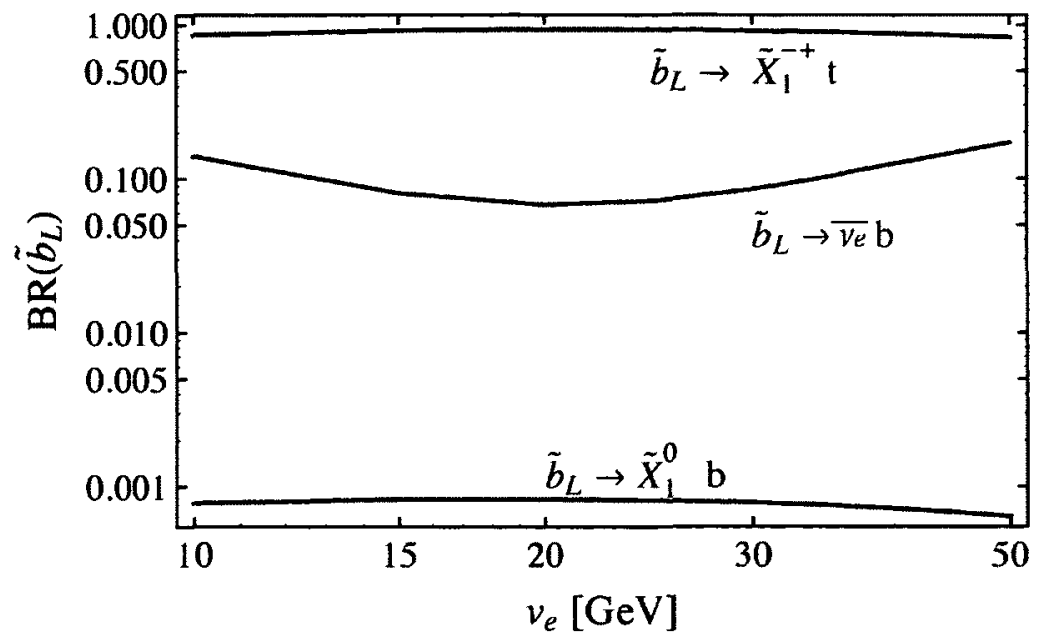

Figure 6.6: Branching fractions for the $\tilde{b}_{L}$ decay modes computed for $\lambda_{S}=\lambda_{T}=0.8$, $\mu=200 \mathrm{GeV}, M_{\tilde{B}}=800 \mathrm{GeV}$ and $M_{\tilde{W}}=1500 \mathrm{GeV}$. We also take $\lambda_{333}^{\prime}=(1.8 \times$ $\left.10^{-2}\right) / y_{b}$, and add together the two neutrino channels $\left(\bar{\nu}_{e}\right.$ and $\left.\bar{\nu}_{\tau}\right)$.

- For the right-handed stop, $\tilde{t}_{R}$, the decay widths are:

$$
\begin{aligned}
\Gamma\left(\tilde{t}_{R} \rightarrow \tilde{X}_{1}^{0} t\right) & =\frac{m_{\tilde{t}_{R}}}{36 \pi}\left(g^{\prime} V_{1 \tilde{b}}^{N}\right)^{2} f_{P H}\left(m_{\tilde{t}_{R}}, m_{\tilde{X}_{1}^{0}}, m_{t}\right), \\
\Gamma\left(\tilde{t}_{R} \rightarrow \tilde{X}_{1}^{+-} b\right) & =\frac{m_{\tilde{t}_{R}}}{8 \pi}\left(y_{t} V_{1 u}^{-}\right)^{2}\left(1-\frac{m_{\tilde{X}_{1}^{0}}^{2}}{m_{\tilde{t}_{R}}^{2}}\right),
\end{aligned}
$$

The dominant decay mode is into $\tilde{X}_{1}^{+-} b$ since it is driven by the top Yukawa coupling, as can be seen in Fig. 6.7. 


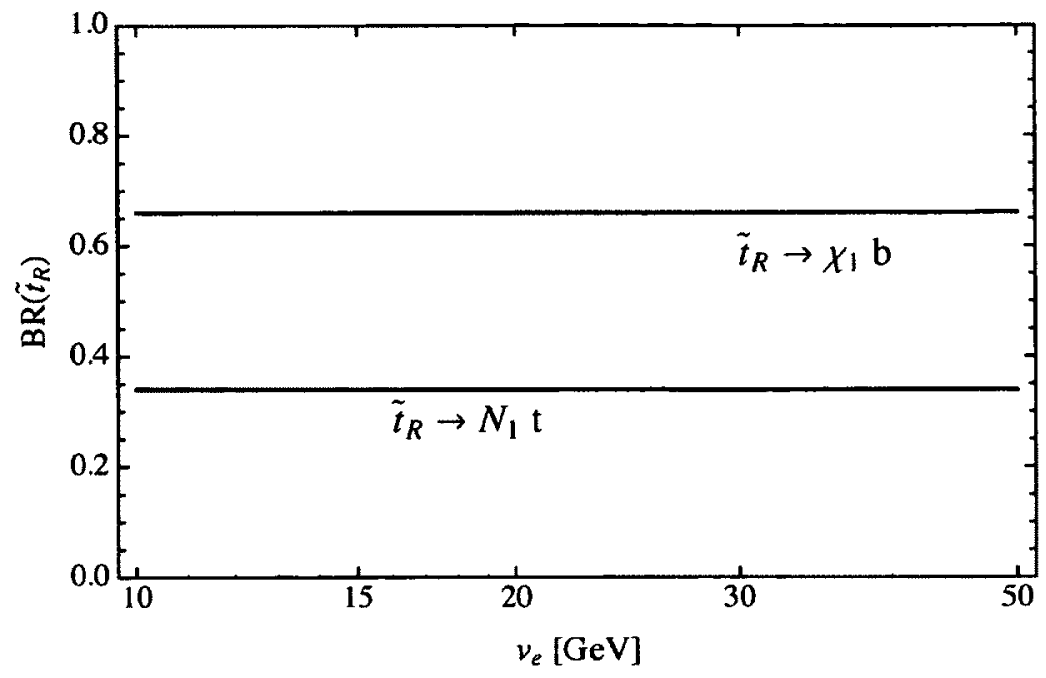

Figure 6.7: Branching ratios for $\tilde{t}_{R}$ decay modes computed for $\lambda_{S}=\lambda_{T}=0.8$, $\mu=200 \mathrm{GeV}, M_{\tilde{B}}=800 \mathrm{GeV}$ and $M_{\tilde{W}}=1500 \mathrm{GeV}$.

- The right-handed sbottom, $\tilde{b}_{R}$, has a variety of decay modes:

$$
\begin{aligned}
\Gamma\left(\tilde{b}_{R} \rightarrow \tilde{X}_{1}^{0} b\right) & =\frac{m_{\tilde{b}_{R}}}{144 \pi}\left(g^{\prime} V_{1 \bar{b}}^{N}\right)^{2}\left(1-\frac{m_{\tilde{X}_{1}^{0}}^{2}}{m_{\tilde{b}_{R}}^{2}}\right), \\
\Gamma\left(\tilde{b}_{R} \rightarrow e_{L}^{-} t\right) & =\frac{m_{\tilde{b}_{R}}}{16 \pi} y_{b}^{2}\left(U_{1 e}^{+}\right)^{2}\left(1-\frac{m_{t}^{2}}{m_{\tilde{b}_{R}}^{2}}\right), \\
\Gamma\left(\tilde{b}_{R} \rightarrow \nu_{e} b\right) & =\frac{m_{\tilde{b}_{R}}}{16 \pi} y_{b}^{2} U_{4 \nu}^{N}, \\
\Gamma\left(\tilde{b}_{R} \rightarrow \tau_{L}^{-} t\right) & =\frac{m_{\tilde{b}_{R}}}{16 \pi}\left(\lambda_{333}^{\prime}\right)^{2}\left(1-\frac{m_{t}^{2}}{m_{\tilde{b}_{R}}^{2}}\right), \\
\Gamma\left(\tilde{b}_{R} \rightarrow \nu_{\tau} b\right) & =\frac{m_{\tilde{b}_{R}}}{16 \pi}\left(\lambda_{333}^{\prime}\right)^{2} .
\end{aligned}
$$

The leptoquarks signals are the dominant ones. Adding the two neutrino channels, the decay mode into $\nu b$ has a branching fraction of about $50 \%$ as shown in Fig. 6.8. The charged lepton signals can involve a LH electron or a $\tau$ plus a top quark. Note also that the decay mode into $\tilde{X}_{1}^{0} b$ is suppressed by a factor 9 . 


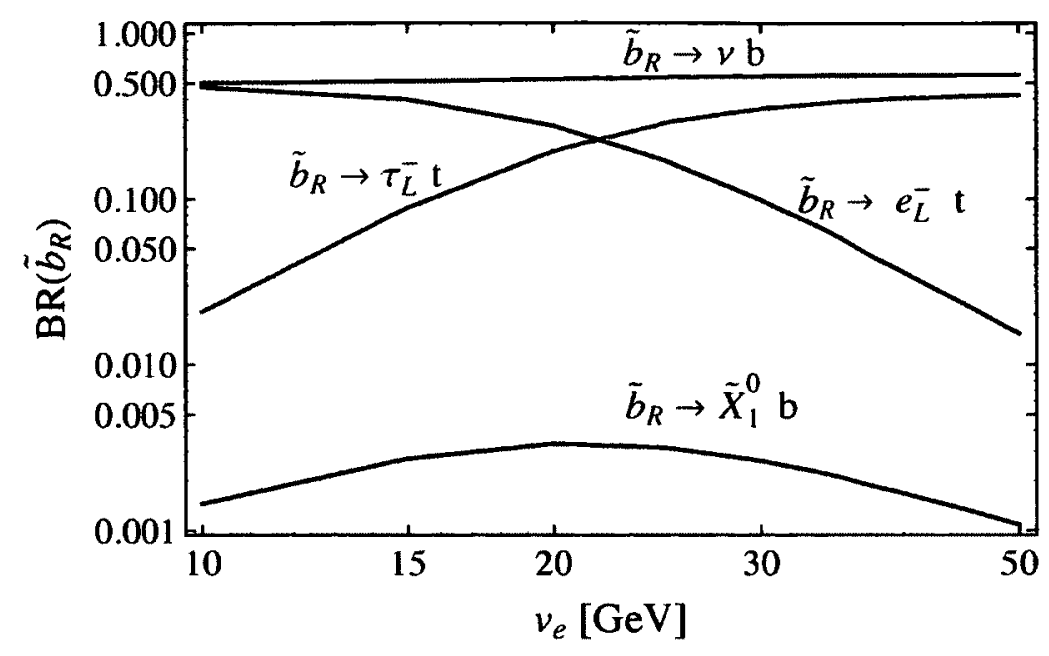

Figure 6.8: Branching ratios for $\tilde{b}_{R}$ decay modes computed for $\lambda_{S}=\lambda_{T}=0.8$, $\mu=200 \mathrm{GeV}, M_{\tilde{B}}=800 \mathrm{GeV}$ and $M_{\tilde{W}}=1500 \mathrm{GeV}$.

\subsection{First and second generation squarks produc- tion}

In the present section we discuss the LHC phenomenology of the first and second generation squarks, which are expected to be the most copiously produced new physics particles.

Although they are not required by naturalness to be light, flavor considerations may suggest that they should not be much heavier than the third generation squarks. Therefore, it is interesting to understand how light these particles could be in our scenario. As we will see, current bounds allow them to be below $1 \mathrm{TeV}$, while in the MSSM the LHC bounds have already exceeded this threshold. The bounds can arise from generic jets $+E_{T}$ searches, as well as from searches involving leptons in the final state. As we will see, the former are more constraining in the majority of the parameter space than the latter for reasons that will be elucidated below. We 
compute the cross section to produce a given final state $X$ in our model as follows:

$$
\sigma(p p \rightarrow X)=\sum_{i} \sigma(p p \rightarrow i) \times \mathrm{BR}(i \rightarrow X),
$$

where $i=\tilde{q}_{1} \tilde{q}_{2}, \tilde{g} \tilde{q}, \tilde{g} \tilde{g}$, and the squark pair production can in principle come in several flavor and chirality combinations. We generate the production cross section for each independent $i$-th state with MadGraph 5 [117] at tree-level. When setting the current bounds, we will also include an estimate for the $K$-factors, as discussed below. Here we note that, due to the assumption of gluinos in the multi- $\mathrm{TeV}$ range, and the fact that we will be interested in squarks below $1 \mathrm{TeV}$, our cross section is largely dominated by squark pair-production. Furthermore, the Dirac nature of the gluinos results in a significant suppression of certain $t$-channel mediated gluino diagrams compared to the Majorana (MSSM) case, as already emphasized in [66] and also as it is shown in Fig. 6.9. Nevertheless, at $M_{\tilde{g}}=2 \mathrm{TeV}$ such contributions are not always negligible, and should be included. For instance, we find that for degenerate squark masses at $m_{\tilde{q}}=800 \mathrm{GeV}$, the production of $\tilde{u}_{L} \tilde{u}_{R}, \tilde{u}_{L} \tilde{d}_{R}$ and $\tilde{u}_{R} \tilde{d}_{L}$ is comparable to the "diagonal" production of $\tilde{q}_{L} \tilde{q}_{L}^{*}$ and $\tilde{q}_{R} \tilde{q}_{R}^{*}$ for all the squark flavors $\tilde{q}=\tilde{u}, \tilde{d}, \tilde{s}, \tilde{c}$ taken together. As indicated in Eq. (6.42) we include separately the BR for each $i$-th state to reproduce $X$, since these can depend on the squark flavor, chirality or generation.

As we have explained in the previous section, all the squarks decay into $\tilde{X}_{1}^{0} j$, with the exception of the left-handed down-type squarks which decay into $\tilde{X}_{1}^{ \pm} j$. The lightest neutralino and chargino decay differently depending on whether the LSP is the neutralino or the LH stau. In the following we will concentrate on the neutralino LSP case.

\subsection{1 $\quad \tilde{X}_{1}^{0}$ LSP topologies}

When the neutralino $\tilde{X}_{1}^{0}$ is the LSP typically its dominant decay mode is into $Z \nu_{e}$ in the large vev limit and into $h \nu_{e}$ in the small vev limit, while the lightest chargino $\tilde{X}_{1}^{ \pm}$ 



Figure 6.9: Left Panel:Squark Anti-Squark production cross section ( for $7 \mathrm{TeV}$ LHC run) computed for a Dirac gluino of mass ( Red) and a Majorana gluino of $M_{\tilde{g}}=2000 \mathrm{GeV}$. The $K$ factor is estimated to be $K \sim 1.6$ In the right panel we computed the ratio between the two cross sections, Dirac and Majorana.

always decays into $W \nu_{e}$. However, as we have explained in the previous section, there is a significant region of the parameter space where the $\tilde{X}_{1}^{0}$ dominant decay mode is into $W e$. Therefore, we will discuss all the three possibilities. First, we will focus on the corresponding simplified models [120] in order to understand which are the most constraining LHC searches for our parameter space, and then we will consider more realistic benchmark points. The scenario we are interested in corresponds to a limit where all the gauginos are decoupled and just the squarks are produced, namely $m_{\tilde{q}} \ll<M_{\tilde{g}}$. The typical simplified models studied by ATLAS and CMS ( [121]) consider instead either $m_{\tilde{q}}=M_{\tilde{g}}$, or $m_{\tilde{q}}>>M_{\bar{g}}$. Therefore, for the moment there is no specific study on our topologies and we will try to adapt studies performed for other scenarios to our case. Since our purpose is to set a rough bound we consider this method satisfactory enough. The mass of the intermediate particle is expressed in terms of the mass of the colored particle produced and of the LSP mass, that is $m_{\text {intermediate }}=x m_{\tilde{q}}+(1-x) m_{L S P}$. The LSP is massless being $\nu_{e}$, and in our scenario $x \sim \mu / m_{\tilde{q}}$ since the intermediate particle is either the lightest neutralino $\tilde{X}_{1}^{0}$ or the 
lightest chargino $\tilde{X}_{1}^{ \pm}$.

Topology (1) : $\tilde{X}_{1}^{0} \rightarrow Z \nu_{e}$

The LHC searches relevant for this topology are:

- jets $+\mathscr{E}_{T}$

- $Z(l l)+\mathrm{jets}+E_{T}$,

- multilepton + jets $+E_{T}$ (without $\mathrm{Z}$ veto)

For the jets and missing energy search there is a CMS study [122] on a simplified model with a similar topology, namely $\tilde{g} \tilde{g}$ production with $\tilde{g} \rightarrow 2 q Z$ and $\chi \rightarrow Z$ LSP. For the moment the limits are set with a luminosity $L=1.1 \mathrm{fb}^{-1}$ and the upper limit on the cross section is always above the production cross section for a $2 \mathrm{TeV}$ gluino. For the moment the most sensitive CMS search for this topology is then the dilepton search $Z(l l)$ jets and $E_{T}[128]$ which has a luminosity study of $4.98 \mathrm{fb}^{-1}$ and consider the same topology. In Fig. 6.10 we compared our cross section for a 2 $\mathrm{TeV}$ Dirac gluino with the upper limit on the cross section for the CMS simplified model assuming the intermediate particle to be $100 \mathrm{GeV}$ and $300 \mathrm{GeV}$. We are more interested to consider a $200 \mathrm{GeV}$ intermediate neutralino $\tilde{X}_{1}^{0}$, therefore we expect our bound to be around $650 \mathrm{GeV}$. In Fig. 6.10 we plotted also the cross section for the MSSM with a $2 \mathrm{TeV}$ gluino to make clear the difference between a Majorana and a Dirac $2 \mathrm{TeV}$ gluino. For the ATLAS searches instead, we apply the $5 \mathrm{fb}^{-1}$ ATLAS search [132] for jets and missing energy. Five different signals region are defined depending on the jets multiplicity. Our topology, and our model in general, is distinguished by long cascade decays, and therefore we should consider the limits on the signal regions characterised by many jets. Each squark indeed decays into 3 jets which are fairly hard if the mass splitting between the squark and $\tilde{X}_{1}^{0}$ is not too 
small. We could have in principle 6 hard jets, we will therefore consider the limits on the signal regions (SR) C to E. A detailed analysis would be required to understand which is the SR more suitable for this topology. Looking at Table 3 in [132] the upper bound on $\sigma \times \epsilon \times A$ is or oder of the few $\mathrm{fb}, \sim 2-4 \mathrm{fb}$. Assuming then $\epsilon \times A \sim 0.1$ (see [137]) we find an estimate for the squark mass upper limit slightly higher than the one set by the dilepton search, $m_{\tilde{q}} \sim 700-800 \mathrm{GeV}$. It is also important to take into account multilepton searches and in Table 6.2 we report the bounds for these searches. However we do not have any simplified model studies applicable to us. Estimating a maximun $\epsilon \times A$ of 0.1 and taking into account the branching ratio suppression we conclude that this search is less sensitive than the dilepton and the jets searches.

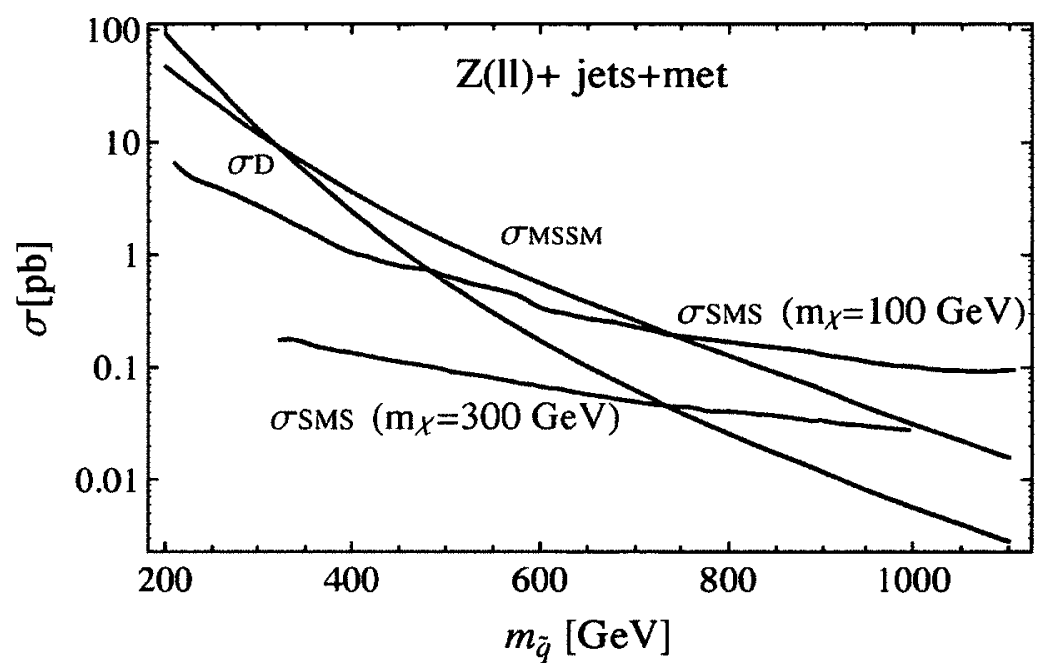

Figure 6.10: Squark squark production cross section computed for a $2 \mathrm{TeV}$ Majorana and Dirac gluino. These are compared on the upper limit on the simplified models cross section for CMS searches assuming an intermediate particle of $100 \mathrm{GeV}$ and 300 $\mathrm{GeV}$. 


\section{Topology (2) : $\tilde{X}_{1}^{0} \rightarrow h \nu_{e}$}

For this scenario the most relevant search is the jets and missing energy [132] since the Higgs decays mostly into hadrons. This topology is characterised by high jets multiplicity and therefore, since there is no dedicated study for it, we can apply the same criteria for the $Z \nu_{e}$ topology to estimate the bound from the $5 \mathrm{fb}^{-1}$ ATLAS search. Therefore, we estimate a mass range of $700-800 \mathrm{GeV}$ for the first and second generation squarks. Also, since the Higgs decays predominantly into $b \bar{b}$, searches with $b$ tagged jets are relevant for this topology. However, for our topology the searches with $b$ tagged jets are less sensitive than the generic jets missing energy searches. Furthermore, the leptonic searches are not relevant for it either due the important suppression from the branching ratio.

\section{Topology (3) : $\tilde{X}_{1}^{0} \rightarrow W e$}

In this case the two relevant searches are: jets and two leptons without missing energy and multileptons, jets and missing energy. The first signal has a branching ratio of $50 \%$ and at the moment there are not relevant searches which look for many jets and two leptons since all the searches have always a cut on the missing transverse energy. It would be then interesting to have a dedicated search for this signal. Therefore, the only searches to apply for the moment are the ATLAS and CMS searches for multilepton signal. The ATLAS limit (with a $Z$ veto) on the BSM cross section is $1.5 \mathrm{fb}[134]$. For the moment the computation of the efficiency times the acceptance for this topology is beyond the scope of this work. Assuming this to be of order of $10^{-1}$ ( see for example [137]) we can set a fairly conservative limit in the mass range $700-800 \mathrm{GeV}$. However, it would be useful to do a more detailed study on the efficiency of the multileptons searches for this topology. 
Topology (4) : $\tilde{X}_{1}^{ \pm} \rightarrow W^{ \pm} \nu_{e}$

In this case the relevant LHC searches are:

- jets $+E_{T}$,

- 1 lepton + jets $+E_{T}$,

- OS 2 leptons $+\mathrm{jets}+E_{T}$.

For the jets plus missing energy search we can apply the same bound as before, and then establish if the bound from the leptonic searches are more stringent. For the one lepton plus jets and missing energy signature ATLAS has set model-independent limits on the cross-section of new physics contributions to the signal regions, which vary between $9 \mathrm{fb}$ and $50 \mathrm{fb}$ depending on the channel and the signal region. Also there is a CMS study on a simplified model [122] which we can compare to our topology. In the CMS simplified model [122] two pair-produced gluinos decay into $q q \chi^{ \pm}$and $q q \chi_{1}^{0}$ respectively. The neutralino $\chi_{1}^{0}$ is the LSP, while $\chi^{ \pm}$decays into $W^{ \pm} \chi_{1}^{0}$. Therefore, as in our scenario, a single lepton is produced via $W$ decay. Looking at Fig.8 in [122] we can see that the upper limit for the cross section is always above the production cross section for our model. For the OS two leptons signal the most stringent limit on the visible cross sections, $\sigma \times \epsilon \times A$, are set by CMS [126] with $4.7 \mathrm{fb}^{-1}$ luminosity. These are in 3-7 fb range, and therefore they give rise to a less stringent limit than the jets plus missing energy search once we take into account the $W$ branching ratio into leptons.

\subsubsection{Realistic benchmark points}

In order to estimate the bounds for our model a study on simplified models is not enough since it is important to take into account different topologies. For example 


\begin{tabular}{|l|l|l|l|}
\hline Search & $\sigma \times a \times \epsilon[f b]$ & $L\left[f b^{-1}\right]$ & Reference \\
\hline 1 lepton & 1.5 & 5 & CMS [127] \\
\hline 2 OS leptons & $3-7$ & 5 & CMS [126] \\
\hline 2 SS leptons & 1.6 & 2.06 & ATLAS [133] \\
\hline$Z\left(l^{+} l^{-}\right)$ & 1.5 & 4.7 & CMS [128] \\
\hline Multilepton ( no $Z)$ & 1.5 & 5 & ATLAS [134] \\
\hline Multilepton $(Z)$ & 3.5 & 5 & ATLAS [134] \\
\hline
\end{tabular}

Table 6.1: The upper limit for $\sigma \times a \times \epsilon$ for all the leptonic channels, the corresponding luminosity and the ATLAS or CMS reference.

the down type left handed squarks decay into charginos and in the intermediate vev region there are different decay modes for $\tilde{X}_{1}^{0}$ with a sizeable branching ratio. A full simulation of our signatures would be required to set precise bounds on the squark masses. However, at least for now, we have already explained that our purpose is to set a rough bound on the squark masses.

We will first consider the benchmark point where the dominant decay modes for $\tilde{X}_{1}^{0}$ are into $Z \nu_{e}$ in the large vev limit and $h \nu_{e}$ in the small vev limit. The LHC searches relevant for this scenario are:

- jets $+E_{T}$,

- single lepton + jets $+E_{T}$,

- OS dilepton $+E_{T}+$ jets ,

- dilepton (from $Z$ decay) + jets $+E_{T}$,

- multilepton + jets $+E_{T}$ without $\mathrm{Z}$ cut.

In Fig. 6.11 the branching ratios for these channels are computed in function of the sneutrino vev $v_{e}$ considering our benchmark point. We notice that even though the dominant topologies are different in the small and in the large vev limit the global 


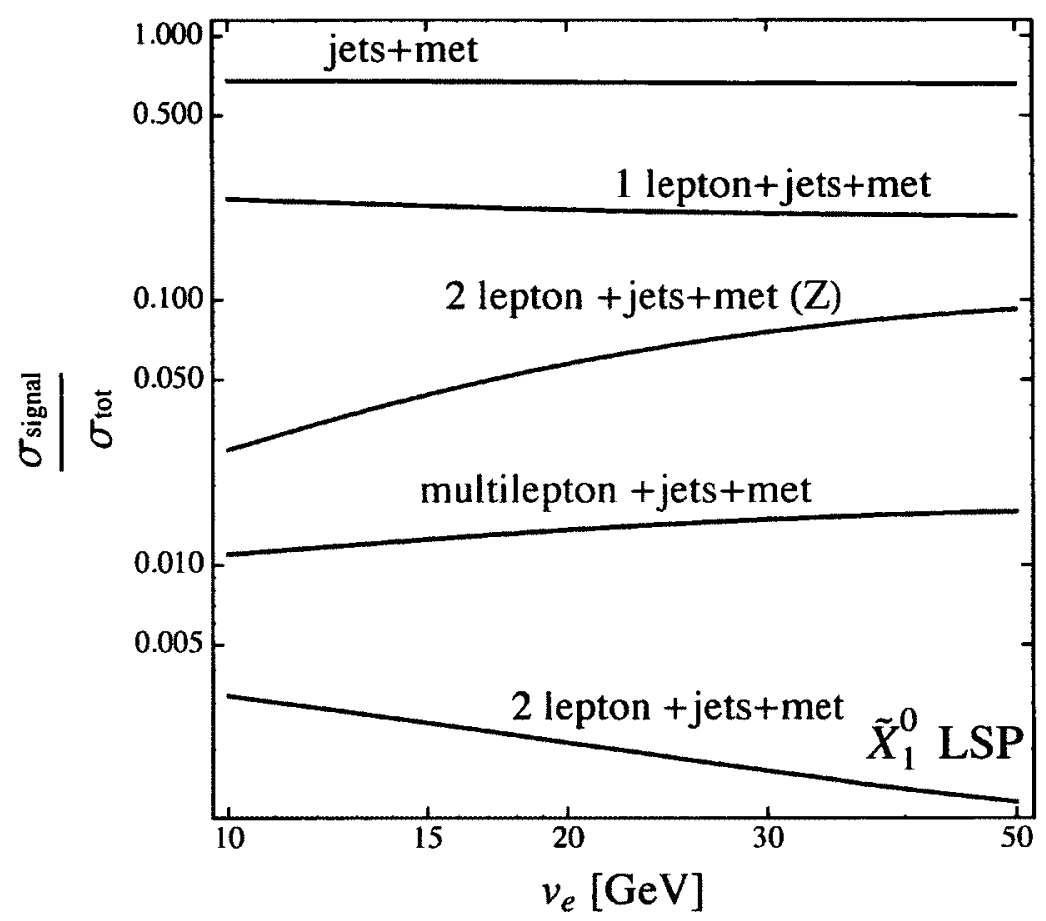

Figure 6.11: Branching ratios for the possible LHC signals computed for $\lambda_{S}=\lambda_{T}=$ $0.8, \mu=-200 \mathrm{GeV}, M_{\tilde{B}}=1000 \mathrm{GeV}$ and $M_{\tilde{W}}=1500 \mathrm{GeV}$.

picture is the same, that is for example the most copious signal is into jets and missing energy. The jets plus missing energy channel is always the dominant final state, and therefore it will be the one to set the upper bound on the squark mass. Indeed, even though the leptonic LHC searches can be potentially more sensitive due to the smaller SM background the BR suppression is such that the most effective search is the ATLAS jets and missing energy search ${ }^{4}$. Therefore, we expect the first and second generation squarks could be as light as $700-750 \mathrm{GeV}$.

Finding squarks lighter than a $1 \mathrm{TeV}$ would be a strong hint for Dirac gauginos. Squarks below this threshold in the jets plus missing energy channel would be allowed in the MSSM only for heavy gluino ( see for example Fig. 6.10 to compare the bound

\footnotetext{
4 which we expect to be confirmed by the CMS $5 \mathrm{fb}^{-1}$ study on the simplified model analogous to our scenario
} 


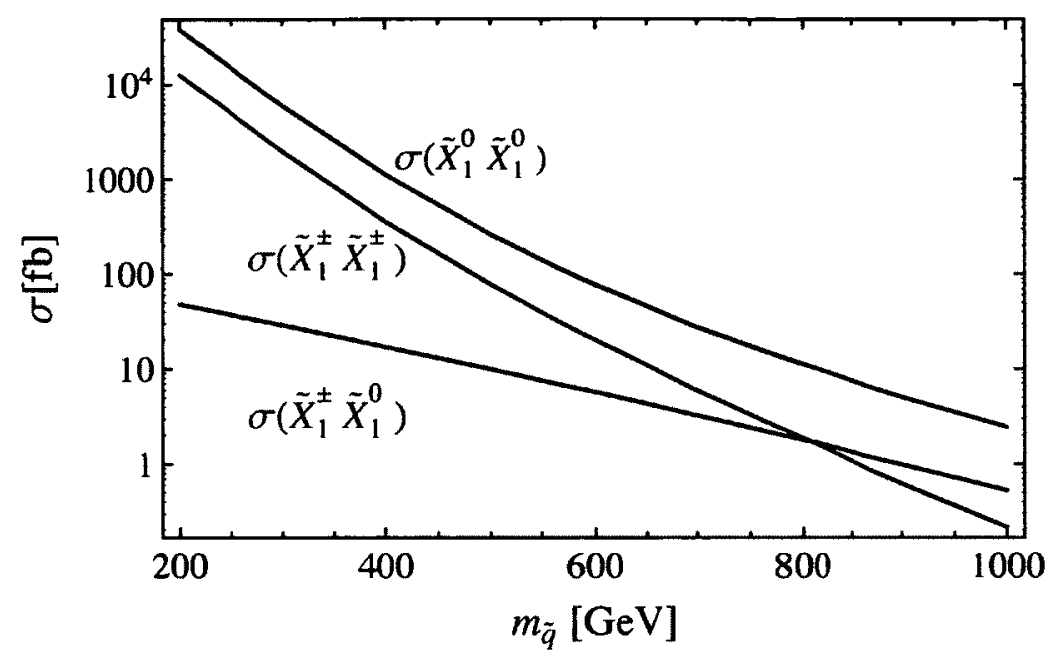

Figure 6.12: Cross section for the separate production of $\tilde{X}_{1}^{0} \tilde{X}_{1}^{0}, \tilde{X}_{0}^{ \pm} \tilde{X}_{0}^{ \pm}$and $\tilde{X}_{1}^{0} \tilde{X}_{0}^{ \pm}$ through squark squark production taking into account the squarks decay modes. This is computed for $m_{\tilde{q}}=700 \mathrm{GeV}$ and $M_{\tilde{g}}=2 \mathrm{TeV}$ for a. $7 \mathrm{TeV} \mathrm{LHC}$ run.

for a $2 \mathrm{TeV}$ Dirac and Majorana gluino) which also would mean a very high fine tuning. We plan to explore this in the near future.

For the other topology we consider a benchmark point where $\mu=200 \mathrm{GeV}$ and $\lambda_{S}=\lambda_{T}=0.4$, while the other parameters are the same. In this case $\tilde{X}_{1}^{0}$ decays dominantly into $W e$ for any values of the sneutrino vev, Fig. 6.3. However, we have to take into account that the left handed down type squarks decay into $\tilde{X}_{1}^{ \pm}$. Therefore, we will consider separately the production of these squarks and the associated production of a down type left handed squark with other squarks. In Fig. 6.12 we showed separately these cross sections. We can see that the $\tilde{X}_{1}^{0} \tilde{X}_{1}^{0}$ is the dominant one, and therefore the stronger bound comes from the searches on multileptons signals. Since the SM background is small the limit on $\sigma \times \epsilon \times A$ is already very low, $1.5 \mathrm{fb}$ with the $Z$ veto. Therefore, assuming $\epsilon \times A \sim 0.1$ cross sections of order of $\sim 10 \mathrm{fb}$ are excluded, and this corresponds to a bound of $700 \mathrm{GeV}$ on the squark mass. We notice that in this case we pay the price of the better sensitivity of the multileptonic 


\begin{tabular}{|l|l|}
\hline Signal & $\sigma \times B R[f b]$ \\
\hline Jets & $21(22)$ \\
\hline Single lepton & $8.43(7.33)$ ) \\
\hline OS dileptons ( via $Z$ decay) & $1.07(3.54)$ \\
\hline OS dileptons (no $Z$ decay) & $0.45(1.48)$ \\
\hline Multileptons & $0.42(0.62)$ \\
\hline
\end{tabular}

Table 6.2: Cross section for all the possible final states for the benchmark point $\mu=-200 \mathrm{GeV}, M_{\tilde{B}}=1 \mathrm{TeV}, M_{\tilde{W}} 1.5 \mathrm{GeV}$ and $\lambda_{S}=\lambda_{T}=0.8$ and $v_{e}=10 \mathrm{GeV}$ (in paranthesis instead the cross section is computed for $v_{e}=50 \mathrm{GeV}$. The squak masses are taken to be $700 \mathrm{GeV}$.

searches since this topology has a very high branching ratio in it. 


\subsection{Conclusions and Outlook}

In the present chapter we discussed the LHC phenomenology of the first and second generation squarks for a $\tilde{X}_{1}^{0}$ LSP. From our qualitative analysis we identify the 700 $800 \mathrm{GeV}$ range as the mass range allowed. This limit is significantly lower than the MSSM bound one for similar topologies ( see [136], [135],and [137]). Furthermore, $700-800 \mathrm{GeV}$ is the same mass range obtained in [66] considering the squark direct decay to the LSP. The reason is that the most sensitive LHC searches at the moment are able to probe cross sections of few tens of $\mathrm{fb}$ and this is the mass range which leads to this cross section for a $2 \mathrm{TeV}$ Dirac gluino. Our result could also apply to other $R$ symmetric models where the LSP is the gravitino instead of the neutrino. This would corresponds to the standard definition of the $R$ symmetry. A LSP gravitino is a well motivated spectrum for $R$-symmetric models since the gravitino is related to $R$ breaking. In order to distinguish our model from other $R$ symmetric models would be then necessary to consider the third generation phenomenology which is instead very particular. We plan in the near future to focus on the this together with the $\tilde{\tau}_{L}$ LSP scenario. The topology $\tilde{X}_{1}^{0} \rightarrow W e$ is instead fairly unique for several reasons. First, we have a striking prediction that we should see just one flavor since the lepton emitted together with the gauge boson is associated to the sneutrino-Higgs. This signal is also present also in models with RPV, but typically the neutralino decay is not prompt as in our case. This, as we have already anticipated in the previous section, is related to the strongest bound on the sneutrino vev in models with a standard lepton number. 


\section{Chapter 7}

\section{Conclusions and Outlooks}

The LHC will soon give an answer to long standing questions in particle physics such as the electroweak symmetry breaking mechanism and the hierarchy problem. If a new particle with the same gauge numbers as the top quark would be discovered, naturalness would be the guiding principle towards the understanding of this discovery. Supersymmetry is one the best motivated solutions to the hierarchy problem. However, the LHC is teaching us that its simplest realisation, the MSSM with $R_{p}$ conservation, might not be the correct SUSY extension of the SM. Therefore, it is important to explore alternatives to the MSSM, and models with Dirac gauginos represent an interesting possibility. In this thesis we have presented a model with Dirac gauginos which posses a $U(1)_{R}$ symmetry identified with the lepton number. This scenario presents a very distinctive phenomenology that we are currently studying with our collaborators. In the future I would be interested in exploring the possibility of using the $R$ symmetry as the main ingredient to build a model of flavorful SUSY breaking mediation. Another interesting question is related to the investigation of the Higgs sector in $R$ symmetric models in order to understand if this symmetry can really play a crucial role in a natural SUSY model.

Little Higgs models is another class of natural models for electroweak symmetry breaking. To improve the agreement of these models with electroweak precision 
measurements, it is useful to impose a discrete symmetry dubbed $T$-parity. However many previous implementations of this symmetry were proven not to be exact, causing all particles of the model to be unstable. The existence of dark matter, as we have briefly explained in the first Chapter, is the most solid evidence for BSM physics, but this does not necessarily call for a solution related to the electroweak scale. However it is interesting and economical to consider whether dark matter may be related to the physics that stabilises the weak scale. A connection between dark matter and physics at the electroweak scale was the main motivation for the little higgs model we presented in Chapter 2. We introduced a new definition of $T$-parity which could be implemented as a symmetry also in an UV completion of the little Higgs model. This implies that the lightest among the new particle introduced is stable and it is a dark matter candidate.

This is a very exciting moment not just because we are revealing the $\mathrm{TeV}$ scale at the LHC, but also because we are investigating the nature of dark matter through many experiments. If the LHC would discover that nature is fine tuned instead, new ideas and priorities would have to guide our research. 


\section{References}

[1] T. Brown, C. Frugiuele and T. Gregoire, JHEP 1106 (2011) 108 [arXiv:1012.2060 [hep-ph]].

[2] C. Frugiuele and T. Gregoire, Phys. Rev. D 85 (2012) 015016 [arXiv:1107.4634 [hep-ph]].

[3] E. Bertuzzo and C. Frugiuele, JHEP 1205 (2012) 100 [arXiv:1203.5340 [hep-ph]].

[4] N. Arkani-Hamed, A. G. Cohen, and H. Georgi, Electroweak symmetry breaking from dimensional deconstruction, Phys. Lett. B513 (2001) 232-240, [hep-ph/0105239].

[5] N. Arkani-Hamed, A. G. Cohen, T. Gregoire, and J. G. Wacker, Phenomenology of electroweak symmetry breaking from theory space, JHEP 08 (2002) 020, [hep-ph/0202089].

[6] N. Arkani-Hamed et. al., The Minimal Moose for a Little Higgs, JHEP 08 (2002) 021, [hep-ph/0206020].

[7] N. Arkani-Hamed, A. G. Cohen, E. Katz, and A. E. Nelson, The littlest Higgs, JHEP 07 (2002) 034, [hep-ph/0206021].

[8] D. E. Kaplan and M. Schmaltz, The little Higgs from a simple group, JHEP 10 (2003) 039, [hep-ph/0302049].

[9] D. B. Kaplan, H. Georgi, and S. Dimopoulos, Composite Higgs Scalars, Phys. Lett. B136 (1984) 187.

[10] M. J. Dugan, H. Georgi, and D. B. Kaplan, Anatomy of a Composite Higgs Model, Nucl. Phys. B254 (1985) 299.

[11] S. Chang and H.-J. He, Unitarity of little Higgs models signals new physics of UV completion, Phys. Lett. B586 (2004) 95-105, [hep-ph/0311177]. 
[12] C. Csaki, J. Hubisz, G. D. Kribs, P. Meade, and J. Terning, Big corrections from a little Higgs, Phys. Rev. D67 (2003) 115002, [hep-ph/0211124].

[13] C. Csaki, J. Hubisz, G. D. Kribs, P. Meade, and J. Terning, Variations of little Higgs models and their electroweak constraints, Phys. Rev. D68 (2003) 035009, [hep-ph/0303236].

[14] T. Gregoire, D. Tucker-Smith, and J. G. Wacker, What precision electroweak physics says about the SU(6)/Sp(6) little Higgs, Phys. Rev. D69 (2004) 115008, [hep-ph/0305275].

[15] C. Kilic and R. Mahbubani, Precision electroweak observables in the minimal moose little Higgs model, JHEP 07 (2004) 013, [hep-ph/0312053].

[16] W. Kilian and J. Reuter, The low-energy structure of little Higgs models, Phys. Rev. D70 (2004) 015004, [hep-ph/0311095].

[17] R. Barbieri, A. Pomarol, R. Rattazzi, and A. Strumia, Electroweak symmetry breaking after LEP-1 and LEP-2, Nucl. Phys. B703 (2004) 127-146, [hep-ph/0405040].

[18] G. Marandella, C. Schappacher, and A. Strumia, Little-Higgs corrections to precision data after LEP2, Phys. Rev. D72 (2005) 035014, [hep-ph/0502096].

[19] Z. Han and W. Skiba, Little Higgs models and electroweak measurements, Phys. Rev. D72 (2005) 035005, [hep-ph/0506206].

[20] H.-C. Cheng and I. Low, TeV symmetry and the little hierarchy problem, JHEP 09 (2003) 051, [hep-ph/0308199].

[21] H.-C. Cheng and I. Low, Little hierarchy, little Higgses, and a little symmetry, JHEP 08 (2004) 061, [hep-ph/0405243].

[22] I. Low, T parity and the littlest Higgs, JHEP 10 (2004) 067, [hep-ph/0409025].

[23] C. T. Hill and R. J. Hill, Topological Physics of Little Higgs Bosons, Phys. Rev. D75 (2007) 115009, [hep-ph/0701044].

[24] C. T. Hill and R. J. Hill, $T^{-}$parity violation by anomalies, Phys. Rev. D76 (2007) 115014, [arXiv:0705.0697].

[25] E. Katz, J.-y. Lee, A. E. Nelson, and D. G. E. Walker, A composite little Higgs model, JHEP 10 (2005) 088, [hep-ph/0312287]. 
[26] I. Low, W. Skiba, and D. Tucker-Smith, Little Higgses from an antisymmetric condensate, Phys. Rev. D66 (2002) 072001, [hep-ph/0207243].

[27] M. Schmaltz and J. Thaler, Collective Quartics and Dangerous Singlets in Little Higgs, JHEP 03 (2009) 137, [arXiv:0812.2477].

[28] A. Hook and J. G. Wacker, Collective Quartics from Simple Groups, JHEP 06 (2010) 041, [arXiv : 0912.0937].

[29] P. Batra and Z. Chacko, Symmetry Breaking Patterns for the Little Higgs from Strong Dynamics, Phys. Rev. D77 (2008) 055015, [arXiv:0710.0333].

[30] M. Piai, A. Pierce, and J. G. Wacker, Composite vector mesons from QCD to the little Higgs, hep-ph/0405242.

[31] D. Krohn and I. Yavin, Anomalies in Fermionic UV Completions of Little Higgs Models, JHEP 06 (2008) 092, [arXiv:0803.4202].

[32] A. Freitas, P. Schwaller, and D. Wyler, A Little Higgs Model with Exact Dark Matter Parity, JHEP 12 (2009) 027, [arXiv:0906. 1816].

[33] C. Csaki, J. Heinonen, M. Perelstein, and C. Spethmann, A Weakly Coupled Ultraviolet Completion of the Littlest Higgs with T-parity, Phys. Rev. D79 (2009) 035014, [arXiv:0804.0622].

[34] D. Pappadopulo and A. Vichi, T-parity, its problems and their solution, arXiv: 1007.4807.

[35] R. Barbieri, L. J. Hall, and V. S. Rychkov, Improved naturalness with a heavy Higgs: An alternative road to LHC physics, Phys. Rev. D74 (2006) 015007, [hep-ph/0603188].

[36] S. R. Coleman and E. J. Weinberg, Radiative Corrections as the Origin of Spontaneous Symmetry Breaking, Phys. Rev. D7 (1973) 1888-1910.

[37] H. Georgi, Vector Realization of Chiral Symmetry, Nucl. Phys. B331 (1990) 311-330.

[38] E. Ma, Verifiable radiative seesaw mechanism of neutrino mass and dark matter, Phys. Rev. D73 (2006) 077301, [hep-ph/0601225].

[39] E. M. Dolle and S. Su, The Inert Dark Matter, Phys. Rev. D80 (2009) 055012, [arXiv:0906.1609]. 
[40] R. Barbieri and A. Strumia, The 'LEP paradox', hep-ph/0007265.

[41] M. E. Peskin and T. Takeuchi, Estimation of oblique electroweak corrections, Phys. Rev. D46 (1992) 381-409.

[42] H. E. Haber, Introductory low-energy supersymmetry, hep-ph/9306207.

[43] J. Hubisz, P. Meade, A. Noble, and M. Perelstein, Electroweak precision constraints on the littlest Higgs model with T parity, JHEP 01 (2006) 135, [hep-ph/0506042].

[44] M. Cirelli, N. Fornengo, and A. Strumia, Minimal dark matter, Nucl. Phys. B753 (2006) 178-194, [hep-ph/0512090].

[45] T. Hambye and M. H. G. Tytgat, Electroweak Symmetry Breaking induced by Dark Matter, Phys. Lett. B659 (2008) 651-655, [arXiv:0707.0633].

[46] L. Lopez Honorez, E. Nezri, J. F. Oliver, and M. H. G. Tytgat, The inert doublet model: An archetype for dark matter, JCAP 0702 (2007) 028, [hep-ph/0612275].

[47] L. Lopez-Honorez and C. E. Yaguna, A new viable region of the inert doublet model, arXiv:1011.1411.

[48] G. Belanger, F. Boudjema, A. Pukhov, and A. Semenov, Dark matter direct detection rate in a generic model with micrOMEGAs2.1, Comput. Phys. Commun. 180 (2009) 747-767, [arXiv:0803.2360].

[49] D. Tucker-Smith and N. Weiner, Inelastic dark matter, Phys. Rev. D64 (2001) 043502, [hep-ph/0101138].

[50] A. Belyaev, C.-R. Chen, K. Tobe, and C. P. Yuan, Phenomenology of littlest Higgs model with $T^{-}$parity: including effects of $T^{-}$odd fermions, Phys. Rev. D74 (2006) 115020, [hep-ph/0609179].

[51] M. S. Carena, J. Hubisz, M. Perelstein, and P. Verdier, Collider signature of T-quarks, Phys. Rev. D75 (2007) 091701, [hep-ph/0610156].

[52] P. Meade and M. Reece, Top partners at the LHC: Spin and mass measurement, Phys. Rev. D74 (2006) 015010, [hep-ph/0601124].

[53] Q.-H. Cao, E. Ma, and G. Rajasekaran, Observing the Dark Scalar Doublet and its Impact on the Standard-Model Higgs Boson at Colliders, Phys. Rev. D76 (2007) 095011, [arXiv:0708.2939]. 
[54] E. Dolle, X. Miao, S. Su, and B. Thomas, Dilepton Signals in the Inert Doublet Model, Phys. Rev. D81 (2010) 035003, [arXiv:0909.3094].

[55] X. Miao, S. Su, and B. Thomas, Trilepton Signals in the Inert Doublet Model, Phys. Rev. D82 (2010) 035009, [arXiv: 1005. 0090].

[56] J. Hubisz, S. J. Lee, and G. Paz, The flavor of a little Higgs with T-parity, JHEP 06 (2006) 041, [hep-ph/0512169].

[57] S. P. Martin, arXiv:hep-ph/9709356.

[58] I. J. R. Aitchison, hep-ph/0505105.

[59] Y. Shirman, arXiv:0907.0039 [hep-ph].

[60] M. Papucci, J. T. Ruderman and A. Weiler, arXiv:1110.6926 [hep-ph].

[61] C. Brust, A. Katz, S. Lawrence and R. Sundrum, JHEP 1203, 103 (2012) [arXiv:1110.6670 [hep-ph]].

[62] L. J. Hall, L. Randall, Nucl. Phys. B352 (1991) 289-308.

[63] L. Randall, N. Rius, Phys. Lett. B286 (1992) 299-306.

[64] P. J. Fox, A. E. Nelson and N. Weiner, JHEP 0208, 035 (2002) [arXiv:hep$\mathrm{ph} / 0206096]$.

[65] M. Heikinheimo, M. Kellerstein and V. Sanz, JHEP 1204 (2012) 043 [arXiv:1111.4322 [hep-ph]].

[66] G. D. Kribs and A. Martin, arXiv:1203.4821 [hep-ph].

[67] S. Abel and M. Goodsell, JHEP 1106 (2011) 064 [arXiv:1102.0014 [hep-th]].

[68] K. Benakli and M. D. Goodsell, Nucl. Phys. B 816, 185 (2009) [arXiv:0811.4409 [hep-ph]].

[69] A. E. Nelson and N. Seiberg, Nucl. Phys. B 416, 46 (1994) [hep-ph/9309299].

[70] G. D. Kribs, E. Poppitz and N. Weiner, Phys. Rev. D 78 (2008) 055010 [arXiv:0712.2039 [hep-ph]].

[71] R. Fok and G. D. Kribs, Phys. Rev. D 82, 035010 (2010) [arXiv:1004.0556 [hep$\mathrm{ph}]$. 
[72] G. D. Kribs, T. Okui and T. S. Roy, Phys. Rev. D 82 (2010) 115010 [arXiv:1008.1798 [hep-ph]].

[73] P. Kumar and E. Ponton, JHEP 1111, 037 (2011) [arXiv:1107.1719 [hep-ph]].

[74] T. Gherghetta, A. Pomarol, Phys. Rev. D67 (2003) 085018. [hep-ph/0302001].

[75] R. Davies, J. March-Russell and M. McCullough, JHEP 1104 (2011) 108 [arXiv:1103.1647 [hep-ph]].

[76] C. S. Aulakh, R. N. Mohapatra, Phys. Lett. B119 (1982) 136.

L. J. Hall, M. Suzuki, Nucl. Phys. B231 (1984) 419. I-H. Lee, Phys. Lett. B138, 121 (1984). J. R. Ellis, G. Gelmini, C. Jarlskog, G. G. Ross, J. W. F. Valle, Phys. Lett. B150 (1985) 142.

G. G. Ross, J. W. F. Valle, Phys. Lett. B151 (1985) 375. S. Dawson, Nucl. Phys. B261 (1985) 297. D. E. Brahm, L. J. Hall, S. D. H. Hsu, Phys. Rev. D42 (1990) 1860-1862. D. -s. Du, C. Liu, Mod. Phys. Lett. A8 (1993) 2271-2276. T. Banks, Y. Grossman, E. Nardi, Y. Nir, Phys. Rev. D52 (1995) 5319-5325. [hep-ph/9505248].

[77] P. Fayet, Nucl. Phys. B90 (1975) 104-124. P. Fayet, Phys. Lett. B64 (1976) 159. P. Fayet, Phys. Lett. B69 (1977) 489. P. Fayet, Phys. Lett. B78 (1978) 417.

[78] R. Barbier et al., Phys. Rept. 420 (2005) 1 [arXiv:hep-ph/0406039].

[79] L. M. Carpenter, arXiv:1007.0017 [hep-th].

[80] K. Benakli and M. D. Goodsell, Nucl. Phys. B 830, 315 (2010) [arXiv:0909.0017 [hep-ph]].

[81] G. Belanger, K. Benakli, M. Goodsell, C. Moura and A. Pukhov, JCAP 0908, 027 (2009) [arXiv:0905.1043 [hep-ph]].

[82] [Particle Data Group], Phys. Lett. B 33 (1970) 1.

[83] A. Pich, NATO Adv. Study Inst. Ser. B Phys. 363 (1997) 173 [arXiv:hep$\mathrm{ph} / 9701263]$.

[84] W. Loinaz, N. Okamura, S. Rayyan, T. Takeuchi, L. C. R. Wijewardhana, Phys. Rev. D70 (2004) 113004. [hep-ph/0403306]. 
[85] H. K. Dreiner, In *Kane, G.L. (ed.): Perspectives on supersymmetry* 462-479. [hep-ph/9707435].

[86] F. Takayama and M. Yamaguchi, Phys. Lett. B 485 (2000) 388 [arXiv:hep$\mathrm{ph} / 0005214]$.

[87] S. Borgani, A. Masiero, M. Yamaguchi, Phys. Lett. B386, 189-197 (1996). [hep$\mathrm{ph} / 9605222]$.

[88] H. Yuksel and M. D. Kistler, Phys. Rev. D 78 (2008) 023502 [arXiv:0711.2906 [astro-ph]].

[89] S. Davidson, M. Losada and A. Riotto, Phys. Rev. Lett. 84 (2000) 4284 [arXiv:hep-ph/0001301].

[90] K. Benakli, M. D. Goodsell, A. -K. Maier, Nucl. Phys. B851 (2011) 445-461. [arXiv:1104.2695 [hep-ph]].

[91] S. D. L. Amigo, A. E. Blechman, P. J. Fox and E. Poppitz, JHEP 0901, 018 (2009) [arXiv:0809.1112 [hep-ph]].

[92] A. E. Nelson, N. Rius, V. Sanz, M. Unsal, JHEP 0208 (2002) 039. [hep$\mathrm{ph} / 0206102]$.

[93] G. F. Giudice, E. W. Kolb and A. Riotto, Phys. Rev. D 64 (2001) 023508 [arXiv:hep-ph/0005123].

[94] G. R. Dvali, G. F. Giudice, A. Pomarol, Nucl. Phys. B478 (1996) 31-45. [hep$\mathrm{ph} / 9603238]$.

[95] A. De Simone, R. Franceschini, G. F. Giudice, D. Pappadopulo and R. Rattazzi, JHEP 1105 (2011) 112 [arXiv:1103.6033 [hep-ph]].

[96] M. Ibe, A. Rajaraman and Z. Surujon, arXiv:1012.5099 [hep-ph].

[97] B. A. Dobrescu and P. J. Fox, Eur. Phys. J. C 70 (2010) 263 [arXiv:1001.3147 [hep-ph]].

[98] A. Freitas, AIP Conf. Proc. 1200 (2010) 446 [arXiv:0909.5308 [hep-ph]].

[99] S. Y. Choi, D. Choudhury, A. Freitas, J. Kalinowski, P. M. Zerwas, Phys. Lett. B697 (2011) 215-221. [arXiv:1012.2688 [hep-ph]].

[100] K. Desch, S. Fleischmann, P. Wienemann, H. K. Dreiner and S. Grab, Phys. Rev. D 83 (2011) 015013 [arXiv:1008.1580 [hep-ph]]. 
[101] J. L. Evans, M. Sudano and T. T. Yanagida, JHEP 1108, 142 (2011) [arXiv:1012.2952 [hep-ph]].

[102] H. K. Dreiner, M. Kramer and B. O'Leary, "Bounds on R-parity violating supersymmetric couplings from leptonic and semi-leptonic meson decays," Phys. Rev. D 75 (2007) 114016 [hep-ph/0612278].

[103] H. K. Dreiner, K. Nickel, F. Staub and A. Vicente, Phys. Rev. D 86, 015003 (2012) [arXiv:1204.5925 [hep-ph]].

[104] G. Arcadi, L. Di Luzio and M. Nardecchia, arXiv:1111.3941 [hep-ph].

[105] A. Kumar, D. Tucker-Smith and N. Weiner, JHEP 1009 (2010) 111 [arXiv:0910.2475 [hep-ph]].

[106] R. Davies and M. McCullough, arXiv:1111.2361 [hep-ph].

[107] T. Gherghetta, G. F. Giudice and J. D. Wells, Nucl. Phys. B 559, 27 (1999) [hep-ph/9904378].

[108] G. Fogli, E. Lisi, A. Marrone, A. Palazzo, and A. Rotunno, "Evidence of $\theta_{13} \neq 0$ from global neutrino data analysis," Phys.Rev. D84 (2011) 053007, arXiv: 1106.6028 [hep-ph].

[109] T. Schwetz, M. Tortola, and J. Valle, "Global neutrino data and recent reactor fluxes: status of three-flavour oscillation parameters," New J.Phys. 13 (2011) 063004, arXiv:1103.0734 [hep-ph].

[110] T. Schwetz, M. Tortola, and J. Valle, "Where we are on $\theta_{-} 13$ : addendum to 'Global neutrino data and recent reactor fluxes: status of three-flavour oscillation parameters'," New J.Phys. 13 (2011) 109401, arXiv:1108.1376 [hep-ph].

[111] K. Abazajian, E. Calabrese, A. Cooray, F. De Bernardis, S. Dodelson, et al., "Cosmological and Astrophysical Neutrino Mass Measurements," Astropart.Phys. 35 (2011) 177-184, arXiv: 1103.5083 [astro-ph.CO].

[112] G. Bhattacharyya, H. Pas, and D. Pidt, "R-Parity violating flavor symmetries, recent neutrino data and absolute neutrino mass scale," Phys.Rev. D84 (2011) 113009, arXiv: 1109.6183 [hep-ph].

[113] F. P. An et al. [DAYA-BAY Collaboration], "Observation of electronantineutrino disappearance at Daya Bay," arXiv:1203.1669 [hep-ex]. 
[114] J. K. Ahn et al. [RENO Collaboration], Phys. Rev. Lett. 108, 191802 (2012) [arXiv:1204.0626 [hep-ex]].

[115] D. Wyler and L. Wolfenstein, Nucl. Phys. B 218, 205 (1983). R. N. Mohapatra and J. W. F. Valle, Phys. Rev. D 34, 1642 (1986). G. C. Branco, W. Grimus and L. Lavoura, Nucl. Phys. B 312, 492 (1989). M.C. Gonzalez-Garcia and J.W.F. Valle, Phys. Lett. B216 (1989) 316. J. Kersten and A. Y. Smirnov, Phys. Rev. D 76, 073005 (2007) arXiv:0705.3221 [hep-ph]. A. Abada, C. Biggio, F. Bonnet, M. B. Gavela and T. Hambye, JHEP 0712 (2007) 061 arXiv:0707.4058 [hep-ph]. M. Shaposhnikov, Nucl. Phys. B 763, 49 (2007) arXiv:hep-ph/0605047. M.B. Gavela, T. Hambye, D. Hernandez and P. Hernandez, JHEP 0909, 038 (2009) arXiv:0906.1461 [hep-ph].

[116] T. Plehn and T. M. P. Tait, J. Phys. G G 36, 075001 (2009) [arXiv:0810.3919 [hep-ph]].

[117] J. Alwall, P. Demin, S. de Visscher, R. Frederix, M. Herquet, F. Maltoni, T. Plehn and D. L. Rainwater et al., JHEP 0709, 028 (2007) [arXiv:0706.2334 [hep-ph]].

[118] P. Fileviez Perez, arXiv:1204.6243 [hep-ph].

[119] C. Kim and T. Mehen, Phys. Rev. D 79, 035011 (2009) [arXiv:0812.0307 [hep$\mathrm{ph}]$.

[120] D. Alves et al. [LHC New Physics Working Group Collaboration], arXiv:1105.2838 [hep-ph].

[121] H. Okawa et al. [ATLAS Collaboration], arXiv:1110.0282 [hep-ex]. CMS-PAS-SUS-11-016

[122] S. Chatrchyan et al. [CMS Collaboration], CMS-PAS-SUS-11-016

[123] S. Chatrchyan et al. [CMS Collaboration], arXiv:1204.3774 [hep-ex].

[124] S. Chatrchyan et al. [CMS Collaboration], arXiv:1207.1798 [hep-ex].

[125] S. Chatrchyan et al. [CMS Collaboration], arXiv:1207.1898 [hep-ex].

[126] S. Chatrchyan et al. [CMS Collaboration], arXiv:1206.3949 [hep-ex].

[127] S. Chatrchyan et al. [CMS Collaboration], JHEP 1108 (2011) 156 [arXiv:1107.1870 [hep-ex]]. 
[128] S. Chatrchyan et al. [CMS Collaboration], arXiv:1204.3774 [hep-ex].

[129] G. Aad et al. [ATLAS Collaboration], Phys. Lett. B 709 (2012) 137 [arXiv:1110.6189 [hep-ex]].

[130] S. Chatrchyan et al. [CMS Collaboration], arXiv:1204.5341 [hep-ex].

[131] G. Aad et al. [ATLAS Collaboration], Phys. Lett. B 710, 67 (2012) [arXiv:1109.6572 [hep-ex]].

[132] G. Aad et al. [ATLAS Collaboration], arXiv:1206.1760 [hep-ex].

[133] G. Aad et al. [ATLAS Collaboration], Phys. Rev. Lett. 108, 241802 (2012) [arXiv:1203.5763 [hep-ex]].

[134] G. Aad et al. [ATLAS Collaboration], ATLAS-CONF-2012-001

[135] J. T. Ruderman and D. Shih, arXiv:1103.6083 [hep-ph].

[136] Y. Kats, P. Meade, M. Reece and D. Shih, JHEP 1202 (2012) 115 [arXiv:1110.6444 [hep-ph]].

[137] K. Rolbiecki and K. Sakurai, arXiv:1206.6767 [hep-ph]. 